ANL-ZPR-482

ANL-ZPR-482

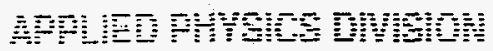

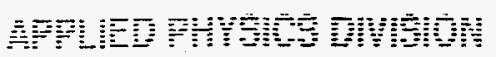

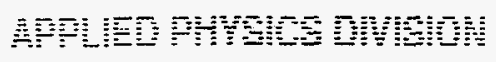

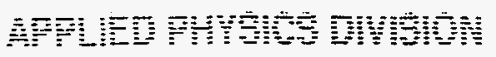

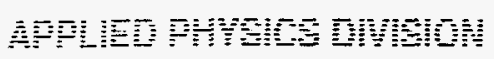

APPLIED PHYSICS DIVISION APPLIED PHYSICS DIVISION APPLIED PHYSICS DIVISION APPLIED PHYSICS DIVISION APPLIED PHYSICS DIVISION APPLIED PHYSICS DIVISION

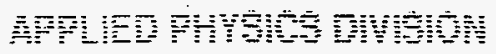

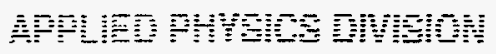

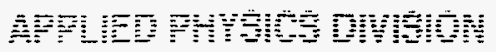

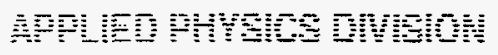

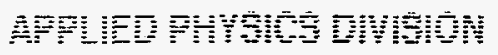
A

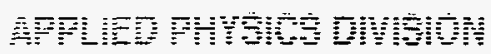

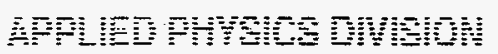

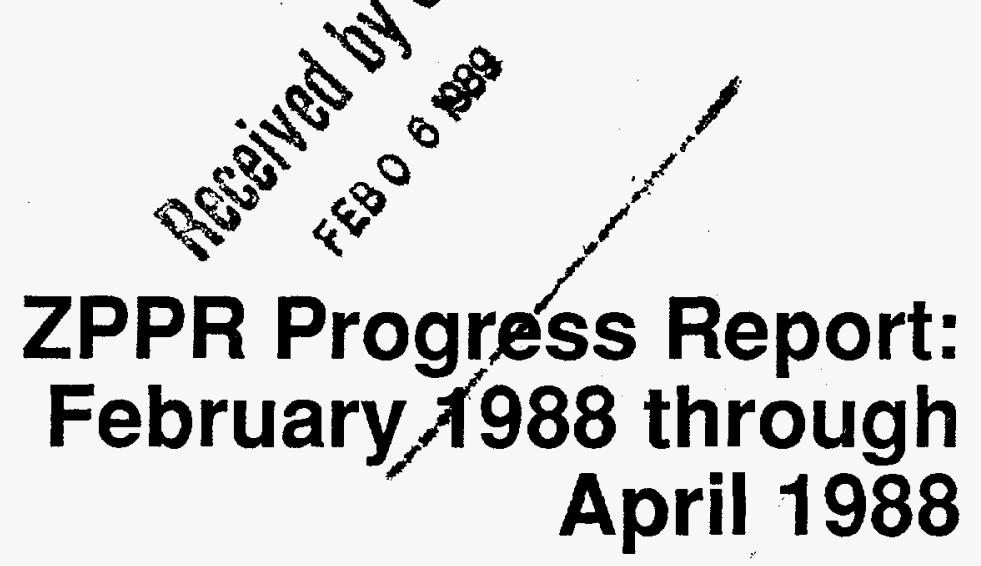
April 1988

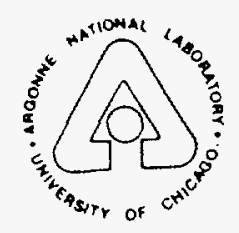

Argonne National Laboratory-West, Idaho Falls, Idaho 83403-2528

Operated by The University of Chicago

for the United States Department of Energy Under Contract W-31-109-Eng-38

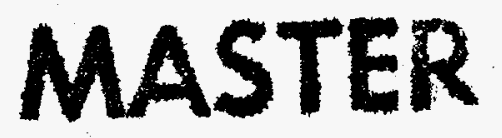

CONTENTS:

ZPPR-17B Control Rods. ZPPR-17C Control Rods. ZPPR-15C Sodium Void. ZPPR-15 High Zr Sodium Void• ZPPR-15 Neutron Spectra•

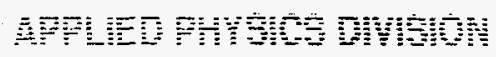

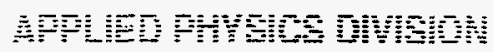

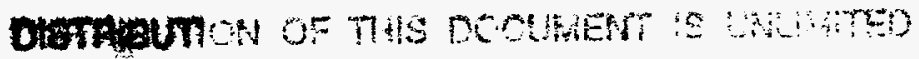
aำ 
Argonne National Laboratory, with facilities in the states of Illinois and Idaho, is owned by the United States government, and operated by The University of Chicago under the provisions of a contract with the Department of Energy.

\section{DISCLAIMER}

This report was prepared as an account of work sponsored by an agency of the United States Government. Neither the United States Government nor any agency thereof, nor any of their employees, makes any warranty, express or implied, or assumes any legal liability or responsibility for the accuracy, completeness, or usefulness of any information, apparatus, product, or process disclosed, or represents that its use would not infringe privately owned rights. Reference herein to any specific commercial product, process, or service by trade name, trademark manufacturer, or otherwise, does not necessarily constitute or imply its endorsement, recommendation, or favoring by the United States Government or any agency thereof. The views and opinions of authors expressed herein do not necessarily state or reflect those of the United States Government or any agency thereof.

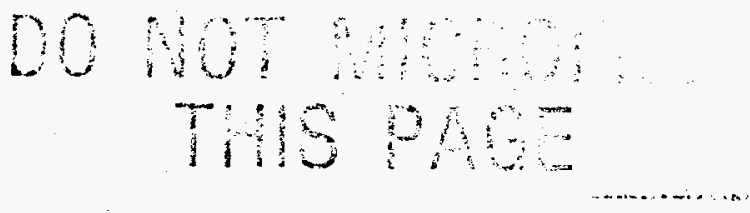




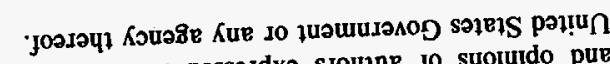

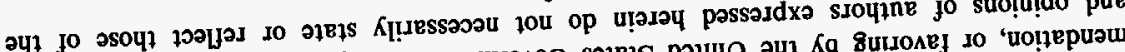
247 jo әsо4

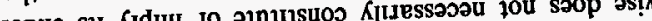

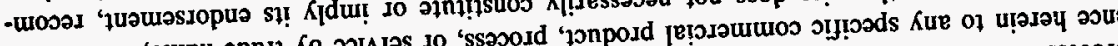

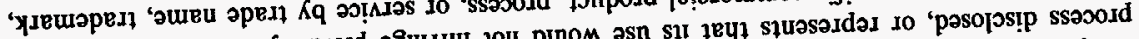

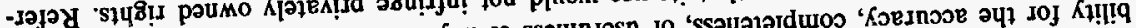
10 '

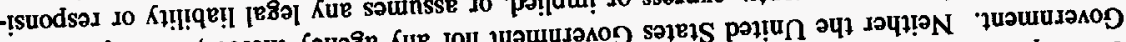

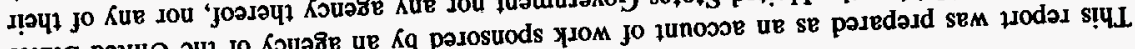

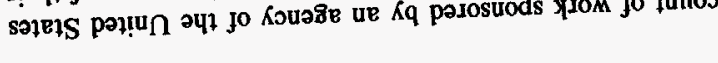

\section{УงWIVTDSIQ}
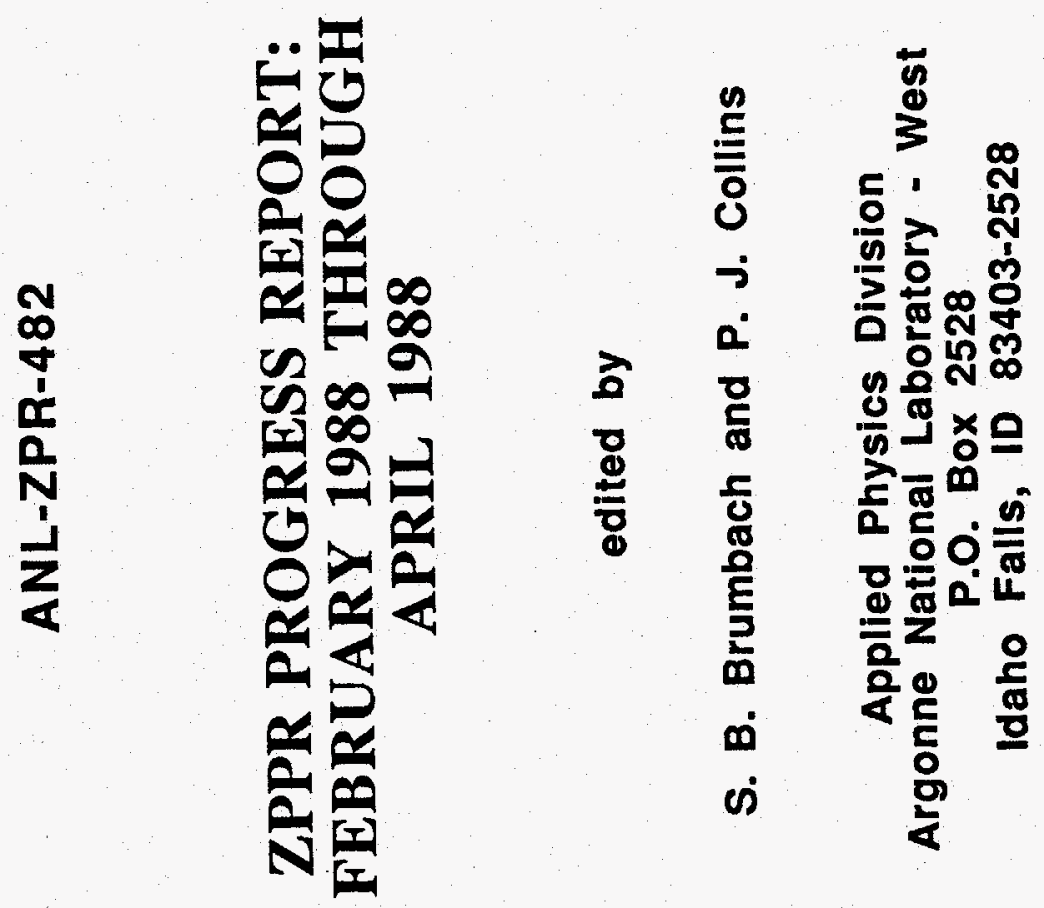
TABLE OF CONTENTS

Page

1. PROGRAM STATUS (S. B. Brumbach, P. J. Collins and D. N. Olsen)

2. MEASUREMENT AND CALCULATION OF CONTROL ROD WORTHS IN ZPPR-17B (F. Nakashima, P. J. Collins

D. M. Smith and G. L. Grasseschi)

3. MEASUREMENT AND CALCULATION OF CONTROL ROD WORTHS IN ZPPR-17C (T. Sanda, P. J. Collins, D. M. Smith and G. L. Grasseschi)

4. REVISION TO THE MCCRUNCH CODE (D. A. Tate and P. J. Collins)

5. REVISIONS TO SODIUM VOID WORTH MEASUREMENTS IN ZPPR-17A (R. W. Goin)

6. MEASUREMENTS AND ANALYSIS OF SODIUM VOID REACTIVITY IN ZPPR-15C (S. B. Brumbach and P. J. Collins)

7. MEASUREMENT AND ANALYSIS OF SODIUM VOID REACTIVITY IN THE HIGH-ZR ZONE OF ZPPR-15 (S. B. Brumbach and P. J. Collins)

8. SUMMARY OF SODIUM VOID RESULTS IN ZPPR-15

9. NEUTRON SPECTRUM MEASUREMENTS IN ZPPR-15

10. CALCULATIONS OF THE CONTROL DRIVELINE EXPANSION SIMULATION IN ZPPR-13D (R. W. Schaefer) 


\section{DISCLAIMER}

Portions of this document may be illegible in electronic image products. Images are produced from the best available original document. 


\title{
ZPPR PROGRESS REPORT: FEBRUARY 1988 THROUGH APRIL 1988
}

\author{
edited by
}

S. B. Brumbach and P. J. Collins

\section{ABSTRACT}

Results are presented for control rod worth experiments in the axially heterogeneous assembly ZPPR-17, a part of the JUPITER-III program.

From the earlier metal-fuel ZPPR-15 program, results are given for measurements and calculations of neutron spectra and sodium voiding in several configurations. 
1. PROGRAM STATUS (S. B. Brumbach, P. J. Collins and D. N. Olsen)

During the February - April 1988 period, the Io portion of the JUPITER-III program was completed. The final configuration was the ZPPR-19B assembly which had uranium and plutonium fuel drawers uniformly mixed in the outer core. Measurements in ZPPR-19B emphasized spatially sensitive parameters aimed at identifying possible effects associated with segregating outer core fuel types as was done in ZPPR-18. The ZPPR-19B experiments included control rod worths, reaction rate distributions, kinetic parameters and measurements of eigenvalue separation.

Following completion of the JUPITER-III program, the ZPPR matrix was completely unloaded in preparation for the ZPPR-20 series of assemblies for the SP-100 space reactor program.

The ZPPR-20 phase A configuration, a mockup of the maximum reactivity configuration of the SP-100, went critical on April 26, 1988 with $167 \mathrm{~kg}$ of ${ }^{235} \mathrm{U}$. The core volume is 36 liters with a diameter and height of about 330 and $406 \mathrm{~mm}$. The configuration has seven simulated internal safety rods withdrawn and the radial reflectors fully open. It is of interest to note that 240 drawer masters and about 1760 drawers were required to bulld phase $A$, which provides an indication of the complexity of the configuration.

The worth of the internal safety rods and the power distribution measurements are in progress.

Several significant additions were made to the ZPPR materials inventory in preparation for the SP-100 Engineering Mockup Criticals (EMC). Newly acquired materials include lithium ( ${ }^{7} \mathrm{Li}$ ), lithium hydride ( $\left.\mathrm{LiH}\right)$, niobium $1 \%$ zirconium $(\mathrm{Nb}-1 \mathrm{Zr})$, core insulation, borated polypropylene and additional polyethylene. In addition, rhenium (Re) was provided by the SP-100 project for temporary use in the critical experiments. Considerable effort was required by ZPPR personnel to prepare these materials for use in the SP-100 EMC. The LiH was granular pellets which were canned in 240 $50.8 \times 50.8 \times 101.6 \mathrm{~mm}$ and $30050.8 \times 50.8 \times 609.6 \mathrm{~mm}$ steel tubes. The ${ }^{7} \mathrm{Li}$ was obtained as ingots and clad in $1506.35 \times 50.8 \times 203.2 \mathrm{~mm}$ stainless 
steel cans. This required removing the ends from stainless-steel void cans, filling the cans by melting the lithium and welding in the end caps. The $\mathrm{Nb}-1 \mathrm{Zr}$ was provided by NASA Lewis Research Center. It was too thick for use and required rolling at ANL-East to obtain a thickness of $3.17 \mathrm{~mm}$, followed by shearing and machining to the final dimensions. The CERA insulation was obtained in rolls and cut into pieces from 76.2 to $203.2 \mathrm{~mm}$ in length. Borated polypropylene was available at the site and a total of $160025.4 \mathrm{x}$ $50.8 \times 304.8 \mathrm{~mm}$ pieces were cut. Polyethylene was also cut into $50.8 \times 50.8$ X 23.2 pieces.

Reference calculations have been completed for all phases of ZPPR-18 and ZPPR-19. Processing of the major series of control rod worth measurements in $\mathrm{ZPPR}-18 \mathrm{~A}$ and $\mathrm{ZPPR}-19 \mathrm{~B}$ has been completed and only a few measurements made in $Z P P R-18 B$ and $Z P P R-19 A$ remain to be analyzed. Experimental data for reaction rates are available for $Z P P R-17 B$ and $Z P P R-17 C$ and will be reported shortly. Processing of foil/cell-average factors required before releasing the $Z P P R-18$ reaction rate data is presently in progress.

The three-dimensional ( $x y z$ geometry) $S_{n}$ code TRITAC has been obtained from OSAKA University. The cross-section homogenization routine, AMSO32, has been modified to provide cross sections is the TRITAC format. Calculations have been made on the Perkin-Elmer computer at ZPPR for the control rod banks in ZPPR-17B. The results show improved consistency with experiment compared with the previous nodal transport calculations. The transport/diffusion correction for the reference $k_{\text {eff }}$ of ZPPR-17 by TRITAC is $+0.6 \% \Delta \mathrm{k}$ and compares well with results of $\mathrm{rz}$ models from the TWODANT code. The nodal transport calculations gave a smaller transport correction of $0.4 \% \Delta \mathrm{k}$. The convergence strategy in TRITAC is not very efficient. A seven group test problem of ZPPR-17 (32 $32 \times 21$ mesh, $\left.s_{4}\right)$ took 45 minutes CPU on the CRAY and about 24 hours on the Perkin-Elmer computer but at present calculations are readily made in free-time on the Perkin-Elmer.

This report contains results from measurements and calculations of control rod worths in $Z P P R-17 B$ and $Z P P R-17 C$. Gamma ray dose results from ZPPR-17A, both measurements and calculations, are being reported separately 
by $\mathrm{H}$. Unesaki in ANL-ZPR-483. Gamma dose measurement results from ZPPR-17B and $17 \mathrm{C}$ are expected to appear in the next progress report.

From the ZPPR-15 assembly, results are reported from sodium void experiments in assembly $15 \mathrm{C}$ and the high-zirconium zone of assembly $15 \mathrm{~B}$. A summary comparing all the sodium void results from the ZPPR-15 assemblies is presented. Also, results of measured neutron spectra from several configurations of $Z P P R-15$ are given with comparisons to calculated spectra. A consistent analysis of smal1-sample worth experiments in ZPPR-15 assemblies $A, B$ and $D$ has been completed and will be issued as ANL-ZPR-486 by R. W. Schaefer. 
2. MEASUREMENT AND CALCULATION OF CONTROL ROD WORTHS IN ZPPR-17B

F. Nakashima,* P. J. Collins, D. M. Smith, and G. L. Grasseschi

\subsection{Introduction}

The ZPPR-17 program was planned to provide basic physics data for an axially - heterogeneous LMR based on configurations of interest to Japanese core-design groups. ZPPR-17B contained a large internal blanket, $0.3 \mathrm{~m}$ thick and $0.87 \mathrm{~m}$ radius, with a single-enrichment fuel zone and a fissile plutonium loading of $2274 \mathrm{~kg}$. The core contained 25 control rod positions (CRPs), arranged as a central CRP, an inner ring of $6 \mathrm{CRPs}$, a middle ring of $6 \mathrm{CRPs}$ and an outer ring of $12 \mathrm{CRPs}$. The outer ring was situated in the fuel region at the periphery of the internal blanket. The principal experiments in ZPPR-17B were measurements of reaction rate distributions and control rod worths.

The control rod locations in ZPPR-17B are shown in Figure 2.1 . The control rod measurements were chosen to cover different options in the core designs. Generally, the central rod plus either the twelve outer ring rods (numbers 14 to 25 ) or six outer ring rods (15 to 25 oddnumbered) were designated as operating (regulating) control rods. The inner ring (numbers 2 to 7 ) were startup rods and the middle ring (numbers 8 to 13) or the middle ring plus the even-numbered rods in the outer ring were the secondary (shutdown) control rods.

A series of twenty control worth measurements were made. These included each of the rod banks, the inner and outer banks halfinserted, eight different combinations of rod banks and six configurations with a rod missing from one of the banks representing stuck rod or rod run-out events.

\footnotetext{
* On assignment from the Power Reactor and Nuclear Fuel Development Corporation (PNC).
} 
A complementary series of control measurements was made in ZPPR-17C. This core was made critical with the central rod and the twelve outer $r$ ing rods half-inserted representing the operating condition at beginning-of-cycle.

\subsection{Description of the Experiments}

The measurements were made in the ZPPR-17B subcritical reference loading described in ANL-ZPR-480, p.3. This reference loading was rebuilt immediately prior to the control rod experiments and the reactivity was established by inverse-kinetics analysis of the power history following rapid insertion of a symmetric bank of ZPPR safety rods. The experimental rod worths were measured relative to this reference by the modified-source-multiplication method using the countrates recorded on a system of sixty-four in-core fission chamber detectors. Fission chambers had dimension 6 in. $x 2$ in. $x 1 / 2$ in. and replaced sodium plates in the normal cells. Counting times varied up to 30 minutes, depending on the subcritical reactivity in each case, and were chosen to give a statistical uncertainty of better than one percent on average.

Each mock-up control rod occupied four matrix positions. Each of the four drawers was fully loaded with $\mathrm{B}_{4} \mathrm{C}$ plates using natural boron (drawer, masters 17-0-603 and 17-0-604). In order to build the required number of rods, both clad and unclad $\mathrm{B}_{4} \mathrm{C}$ plates were used. The compositions of the control rods, with axial sections labeled in inches, are given in Table 2.1. Master 604 was used in the central control rod position and master 603 was used in all other positions.

The measurements were made between May 11, 1987 and May 21, 1987 in reactor loadings 89 to 111 , reactor runs 98 to 122 . The data were recorded on files 94 to 115 of the ZPPR-17 64-detector database. Details of the data processing are given in Section X.4. 


\subsection{Description of the Calculations}

Calculations of detector efficiencies and effective source ratios required for processing experimental data may usually be made to sufficient accuracy by two-dimensional xy models. However, the internal blanket of ZPPR-17 caused strong variations of the axial buckling as a function of radius, especially around the edge of the internal blanket. To avoid complication of the model in this case, all calculations were made with a three-dimensional xyz model with the cross sections collapsed to six groups.

The calculation model for $Z P P R-17 B$ is given in ANL-ZPR-480, p. 9. Collapse of the ENDF/B-V.2 data was made from an xyz model calculated with 21 groups. Cross sections in six groups were obtained for the internal blanket, for single-and double-column-fuel drawers in the inner core (defined as the fuel region above and below the internal blanket) and in the outer core, for the radial blanket, radial reflector, axial blanket, and axial reflector. Cross sections for CRPs were also obtained with this model. A second calculation with the center control rod and the outer control rods inserted was used to collapse data for the control rods. Group boundaries for the six group set were the same as used for ZPPR-17A (ANL-ZPR-476, Table 3.1, p. 21).

The calculations were made in $x y z$ geometry with full-xy/ half $-z$, quarter-xy/half $-z$, and quarter-xy/full-z symmetric models as required to represent the geometry of the control rod patterns. For the subcritical states both an adjoint flux for the homogenous problem and a source-driven real flux were required to calculate detector efficiencies and effective source ratios. All calculations were made with the finite-difference solution of D1F3D since an initial test of the source method in nodal diffusions lead to convergence problems. The calculation of detector efficiencies and effective source ratios is described in ANL-ZPR-480, p. 20 et. seq. 


\subsection{Details of the Experimental Data Processing}

A summary of the data processing using the MCCRUNCH code is given in Table 2.2. The results are listed in the order of the measurements. A shorthand notation ( $a, b, c, d$ ) is used to describe the number of control rods in the center position, the inner ring, the middle ring and the outer ring. These rings are also abbreviated in the next section to $I R, M R$, and $O R$. Where necessary the letter $H$ is used to indicate rods half inserted, viz $6 \mathrm{H}$, and $\emptyset$ is used to indicate odd numbered rods. Worths are given as positive quantities for simplicity.

The reference reactivity for the series was $-21.86 \phi$ and was measured by inverse-kinetics analysis of a rod drop immediately before the worth measurements. The 64-detector data for this reference were recorded on data File 94.

The analysis in Table 2.2 is shown for the three passes through MCCRUNCH (a) using the mean of all results (b) rejecting detectors which are more than 3.60 (counter statistics) from the mean, and (c) least squares fitting of reactivity versus detector efficiency.

Detectors falling Immediately adjacent to control rods, i.e. those in the neighboring drawers, were excluded from the MCCRUNCH analysis since the diffusion calculations do not provide accurate efficiency estimates for these cases. No more then 10 detectors were excluded throughout the series.

As expected, in a moderately sensitive core, the LSFIT method produces the best $f$ it to the experimental countrate ratios. The revised LSFIT method, described in Section 4, was used in this analysis.

\subsection{Recommended Worths and Comparison with Calculation}

The recommended worths and uncertainties are those from the LSFIT analysis of Table 2.2. The results are given in Tables $2.3,2.4$, 
and 2.5 , grouped into rod banks, rod bank combinations and banks with missing rods.

The calculated results were obtained with the finitedifference $x y z$ models and 6 group cross sections. The k-effective value for the subcritical reference core $\left(k_{0}\right)$ was 0.985234 . Calculated worths are defined as $\Delta k /\left(k_{0} k_{1} \beta\right)$ with $\beta$-effective $=0.3374 \%$.

The C/E results are in the range of 0.95 (center rod) to 1.00 (outer ring half-inserted). No clear trend in $\mathrm{C} / \mathrm{E}$ as a function of radius emerges from the present analysis. The center rod is predicted $2-3 \%$ lower than the inner ring $(C / E=0.98)$ the middle $\mathrm{ring} C / \mathrm{E}$ is 0.97 and the outer ring $\mathrm{C} / \mathrm{E}$ is 0.99 .

Predictions for rod banks combinations and banks with missing rods are consistent with those for the individual banks with $\mathrm{C} / \mathrm{E}$ values in the range 0.97 to 1.00 .

The ratios of worths for rod banks half-inserted to the worths of the fully inserted banks are quite interesting:

$\begin{array}{lll}\text { inner ring } & \begin{array}{l}\text { measured } \\ \text { calculated }\end{array} & 0.545 \\ & & \\ & & \\ \text { outer ring } & \text { measured } & 0.542 \\ & \text { calculated } & 0.482\end{array}$

Thus, the rods half-inserted in the internal blanket are worth $9 \%$ more than half of the worth when fully-inserted while at the periphery of the blanket they are worth $4 \%$ less than half of the worth when fullyinserted. Experiment and calculation agree closely for these ratios.

Some rod interaction effects are shown in Table 2.6 which compares the worth of the center rod and of different banks of rods when inserted alone or with other rod banks inserted in the core. These worths were 
obtained by subtraction of the worths in the previous tables, hence the statistical uncertainties increase. The worth of the center rod, $1 \$$ when inserted alone, varies from $0.22 \$$ with the inner and middle rings inserted to $2.14 \$$ with the outer ring inserted.

The worths of withdrawal of single rods from a bank in different situations are compared in Table 2.7. Since the single rod worths were not measured, the average worth of the rods in the appropriate banks are given for comparison. The worths of the withdrawn rods vary considerably: the worth of CR2 in the inner bank is $0.46 \$$ when the center and middle banks are inserted and $0.94 \$$ when the center and 6 outer bank rods are inserted compared with the mean worth in the bank of 0.97 \$. The worth of $\mathrm{CR} 14$ in the outer bank (with center rod also inserted) is $4.1 \$$ compared with the mean worth in the outer bank of 1.9\$. These interaction effects are calculated within $10 \%$ by diffusion theory.

Calculations for the symmetric rod banks have been made by nodal diffusion (NDT) and nodal transport (NTT) models. The results are compared with the finite difference diffusion (FDDT) values, using a $55 \mathrm{~mm}$ mesh size, in Table 2.8. Mesh and transport effects are quite different to those found in conventional and radially-heterogeneous cores. Mesh corrections are relatively large - from $+8 \%$ to $+12 \%-$ while the transport correction is near zero for the central rod increasing with radius to $-5 \%$ for the outer rod bank. The calculated worths are notably higher $(6 \%$ to $9 \%$ ) by nodal transport calculation then the original finite difference diffusion.

The nodal transport $C / E$ results show no clear trend with radius but the center rod and the middle bank have $\mathrm{C} /$ Es about $1 \%$ lower than those for the inner bank and the outer bank. The $\mathrm{C} / \mathrm{E}$ results for bank combinations fall between those for the individual banks. The spread in $C / E$ values (1.041 to 1.057) is less than half of the spread with the finite difference diffusion calculations. 


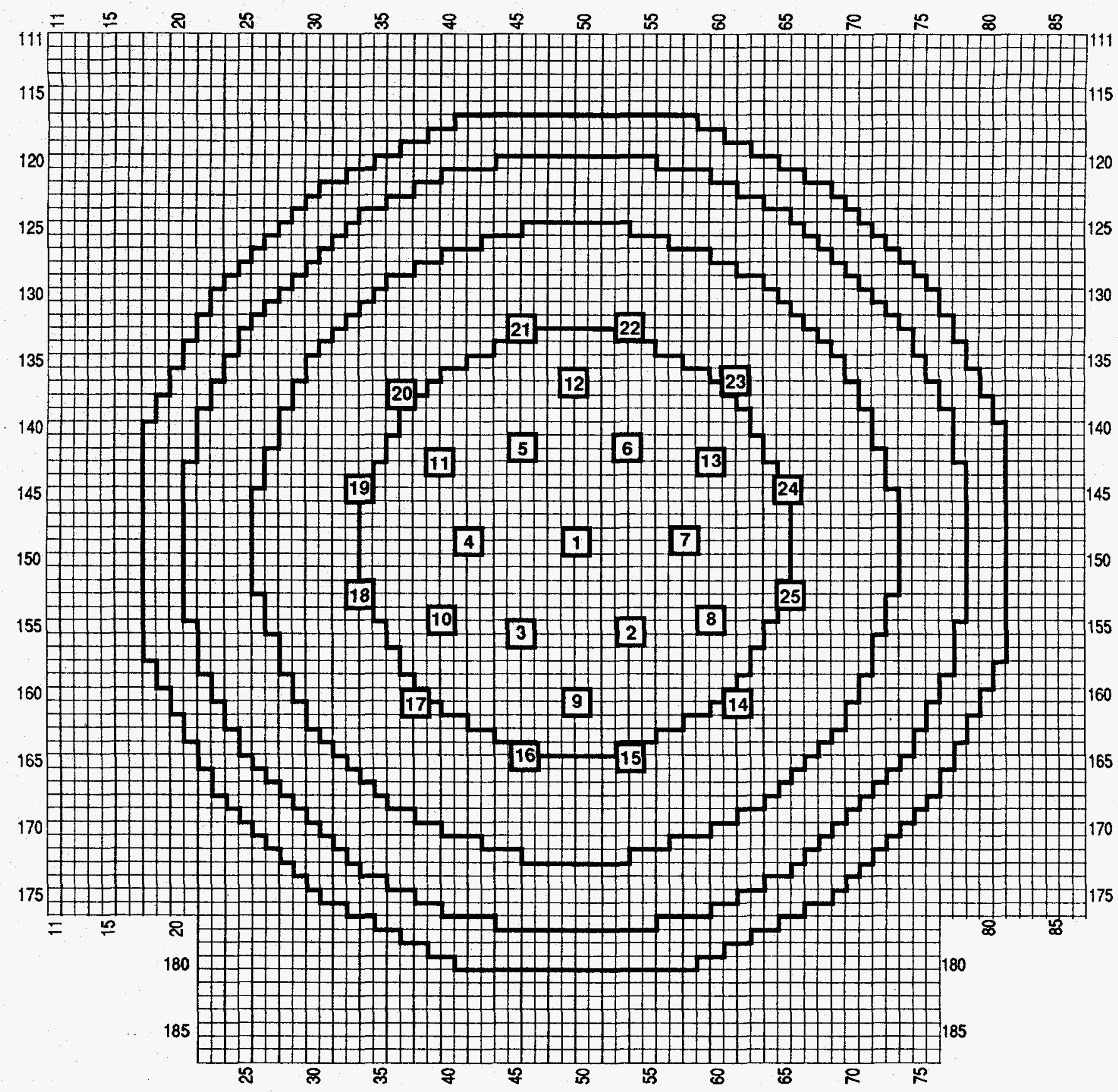

Fig. 2.1. Control Rod Locations in ZPPR-17B 
TABLE 2.1 Control Rod Compositions for ZPPR-17B (atoms/barn-cm)

\begin{tabular}{|c|c|c|c|c|}
\hline Isotope & $\begin{array}{c}\text { Master } \\
603 \\
0-6 \\
\end{array}$ & $\begin{array}{c}\text { Master } \\
603 \\
6-20 \\
\end{array}$ & $\begin{array}{c}\text { Master } \\
603 \\
20-31 \\
\end{array}$ & $\begin{array}{c}\text { Master } \\
603 \\
31-36 \\
\end{array}$ \\
\hline$B-10$ & 0.0158704 & 0.0151769 & -- & -- \\
\hline$B-11$ & 0.0643536 & 0.0615467 & $\cdots$ & $\cdots$ \\
\hline $\mathrm{C}$ & 0.0203704 & 0.0197666 & 0.0000308 & 0.0000447 \\
\hline 0 & 0.0001353 & 0.0000534 & 0.0000013 & 0.0000013 \\
\hline $\mathrm{Na}$ & --- & --- & 0.0181363 & 0.0176957 \\
\hline Si & 0.0002522 & 0.0002235 & 0.0001651 & 0.0001707 \\
\hline $\mathrm{A} 1$ & 0.0000021 & 0.0000028 & 0.0000043 & 0.0000042 \\
\hline $\mathrm{Mn}$ & 0.0002004 & 0.0002204 & 0.0002432 & 0.0002501 \\
\hline $\mathrm{Cr}$ & 0.0023506 & 0.0026105 & 0.0029562 & 0.0030295 \\
\hline $\mathrm{Fe}$ & 0.0084649 & 0.0093681 & 0.0104363 & 0.0109693 \\
\hline $\mathrm{Ni}$ & 0.0010327 & 0.0011621 & 0.0013233 & 0.0013539 \\
\hline $\mathrm{Cu}$ & 0.0000314 & 0.0000325 & 0.0000357 & 0.0000374 \\
\hline Mo & 0.0000157 & 0.0000162 & 0.0000174 & 0.0000183 \\
\hline $\mathrm{P}$ & 0.0000040 & 0.0000040 & 0.0000040 & 0.0000042 \\
\hline $\mathrm{S}$ & 0.0000013 & 0.0000012 & 0.0000012 & 0.0000013 \\
\hline $\mathrm{Cl}$ & --- & $--\infty$ & 0.0000006 & 0.0000006 \\
\hline $\mathrm{Ca}$ & $\ldots$ & $--\infty$ & 0.0000042 & 0.0000041 \\
\hline Co & 0.0000040 & 0.0000037 & 0.0000037 & 0.0000043 \\
\hline
\end{tabular}


TABLE 2.1. (contd)

\begin{tabular}{|c|c|c|c|c|}
\hline Isotope & $\begin{array}{c}\text { Master } \\
604 \\
0-6 \\
\end{array}$ & $\begin{array}{c}\text { Master } \\
604 \\
6-20 \\
\end{array}$ & $\begin{array}{c}\text { Master } \\
604 \\
20-31 \\
\end{array}$ & $\begin{array}{c}\text { Master } \\
604 \\
31-36 \\
\end{array}$ \\
\hline$B-10$ & 0.0158704 & 0.0151625 & $-\cdots$ & --- \\
\hline$B-11$ & 0.0643536 & 0.0614842 & --- & --- \\
\hline C & 0.0203704 & 0.0197463 & 0.0000308 & 0.0000447 \\
\hline 0 & 0.0001353 & 0.0000534 & 0.0000013 & 0.0000013 \\
\hline $\mathrm{Na}$ & -- & -- & 0.0181363 & 0.0176957 \\
\hline $\mathrm{Si}$ & 0.0002522 & 0.0002235 & 0.0001651 & 0.0001707 \\
\hline$A 1$ & 0.0000021 & 0.0000028 & 0.0000043 & 0.0000042 \\
\hline $\mathrm{Mn}$ & 0.0002004 & 0.0002204 & 0.0002432 & 0.0002501 \\
\hline $\mathrm{Cr}$ & 0.0023506 & 0.0026105 & 0.0029562 & 0.0030295 \\
\hline $\mathrm{Fe}$ & 0.0084649 & 0.0093681 & 0.0104363 & 0.0109693 \\
\hline $\mathrm{Ni}$ & 0.0010327 & 0.0011621 & 0.0013233 & 0.0013539 \\
\hline $\mathrm{Cu}$ & 0.0000314 & 0.0000325 & 0.0000357 & 0.0000374 \\
\hline Mo & 0.0000157 & 0.0000162 & 0.0000174 & 0.0000183 \\
\hline$P$ & 0.0000040 & 0.0000040 & 0.0000040 & 0.0000042 \\
\hline$S$ & 0.0000013 & 0.0000012 & 0.0000012 & 0.0000013 \\
\hline $\mathrm{Cl}$ & --- & $-\infty$ & 0.0000006 & 0.0000006 \\
\hline $\mathrm{Ca}$ & $\cdots$ & - & 0.0000042 & 0.0000041 \\
\hline Co & 0.0000040 & 0.0000037 & 0.0000037 & 0.0000043 \\
\hline
\end{tabular}


TABLE 2.2 Data Processing for ZPPR-17B Control Rods

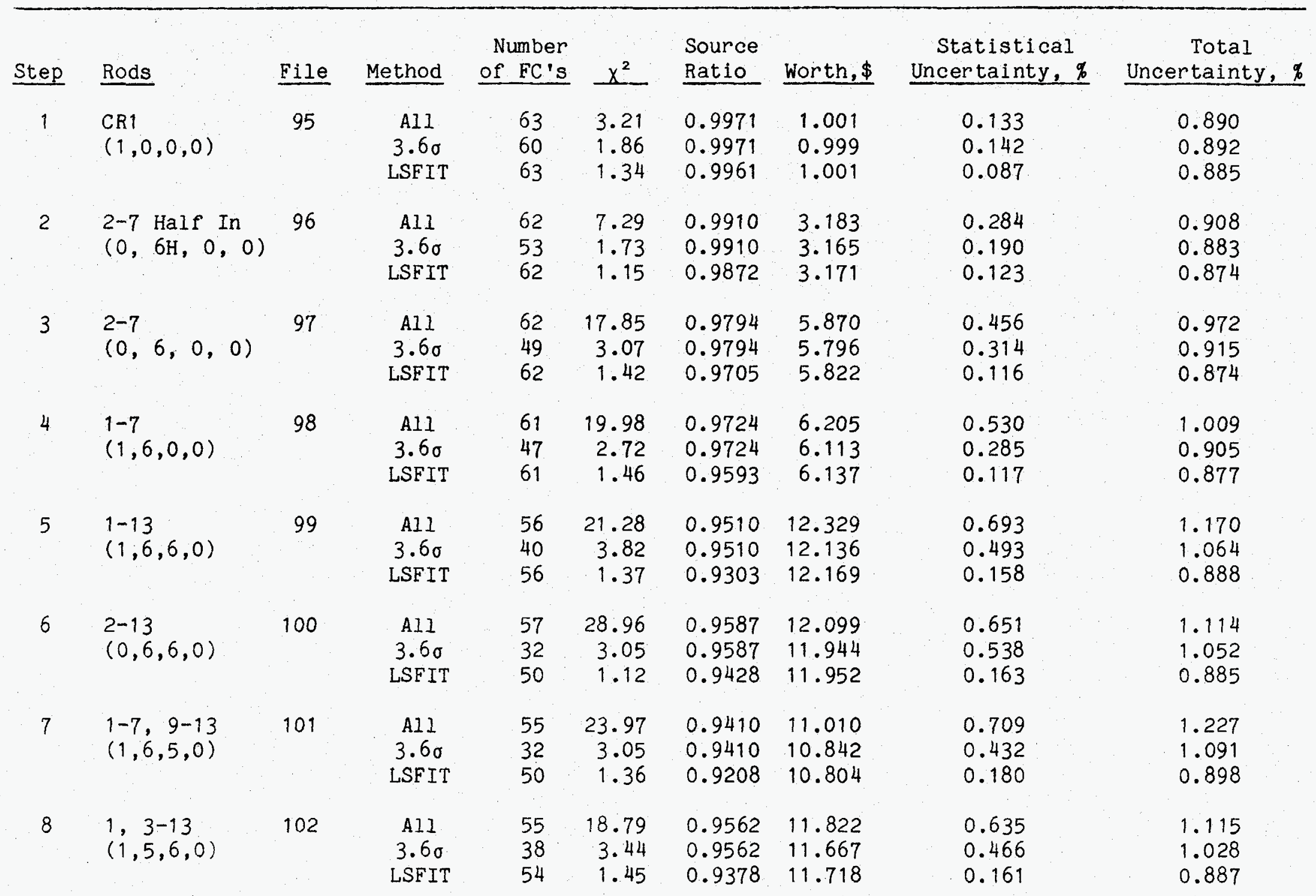


TABLE 2.2 (contd)

\begin{tabular}{|c|c|c|c|c|c|c|c|c|c|}
\hline Step & Rods & File & Method & $\begin{array}{r}\text { Number } \\
\text { of FC's } \\
\end{array}$ & $x^{2}$ & $\begin{array}{l}\text { Source } \\
\text { Ratio } \\
\end{array}$ & Worth, $\$$ & $\begin{array}{c}\text { Statistical } \\
\text { Uncertainty, }\end{array}$ & $\begin{array}{c}\text { Total } \\
\text { Uncertainty, } \% \\
\end{array}$ \\
\hline 9 & $\begin{array}{l}1,8-13 \\
(1,0,6,0)\end{array}$ & 103 & $\begin{array}{l}\text { All } \\
3.60 \\
\text { LSFIT }\end{array}$ & $\begin{array}{l}58 \\
47 \\
58\end{array}$ & $\begin{array}{l}9.05 \\
2.69 \\
1.44\end{array}$ & $\begin{array}{l}0.9877 \\
0.9877 \\
0.9832\end{array}$ & $\begin{array}{l}9.010 \\
8.926 \\
9.017\end{array}$ & $\begin{array}{l}0.332 \\
0.320 \\
0.126\end{array}$ & $\begin{array}{l}0.920 \\
0.915 \\
0.870\end{array}$ \\
\hline 10 & $\begin{array}{l}8-13 \\
(0,0,6,0)\end{array}$ & 104 & $\begin{array}{l}\text { AII } \\
3.6 \sigma \\
\text { LSFIT }\end{array}$ & $\begin{array}{l}59 \\
52 \\
59\end{array}$ & $\begin{array}{l}5.71 \\
3.59 \\
1.25\end{array}$ & $\begin{array}{l}0.9953 \\
0.9953 \\
0.9937\end{array}$ & $\begin{array}{l}8.304 \\
8.273 \\
8.318\end{array}$ & $\begin{array}{l}0.259 \\
0.409 \\
0.121\end{array}$ & $\begin{array}{l}0.896 \\
0.950 \\
0.867\end{array}$ \\
\hline 11 & $\begin{array}{l}9-13 \\
(0,0,5,0)\end{array}$ & 105 & $\begin{array}{l}\text { AII } \\
3.60 \\
\text { LSFIT }\end{array}$ & $\begin{array}{l}58 \\
49 \\
50\end{array}$ & $\begin{array}{l}6.64 \\
2.65 \\
0.99\end{array}$ & $\begin{array}{l}0.9782 \\
0.9782 \\
0.9729\end{array}$ & $\begin{array}{l}6.571 \\
6.549 \\
6.537\end{array}$ & $\begin{array}{l}0.256 \\
0.298 \\
0.134\end{array}$ & $\begin{array}{l}0.896 \\
0.907 \\
0.873\end{array}$ \\
\hline 12 & $\begin{array}{l}1,3-7,15, \\
17,19,21,23,25 \\
(1,5,0,60)\end{array}$ & 106 & $\begin{array}{l}\text { AlI } \\
3.66 \\
\text { LSFIT }\end{array}$ & $\begin{array}{l}56 \\
42 \\
51\end{array}$ & $\begin{array}{l}16.28 \\
3.19 \\
1.46\end{array}$ & $\begin{array}{l}0.9848 \\
0.9848 \\
0.9848\end{array}$ & $\begin{array}{l}17.413 \\
17.229 \\
17.321\end{array}$ & $\begin{array}{l}0.487 \\
0.392 \\
0.131\end{array}$ & $\begin{array}{l}0.985 \\
0.942 \\
0.870\end{array}$ \\
\hline 13 & $\begin{array}{l}2-7,15,17 \\
19,21,23,25 \\
(0,6,0,60)\end{array}$ & 107 & $\begin{array}{l}\text { AII } \\
3.60 \\
\text { LSFIT }\end{array}$ & $\begin{array}{l}57 \\
36 \\
48\end{array}$ & $\begin{array}{r}17.52 \\
4.39 \\
1.07\end{array}$ & $\begin{array}{l}0.9904 \\
0.9904 \\
0.9869\end{array}$ & $\begin{array}{l}17.938 \\
17.770 \\
17.856\end{array}$ & $\begin{array}{l}0.478 \\
0.570 \\
0.147\end{array}$ & $\begin{array}{l}0.960 \\
1.028 \\
0.871\end{array}$ \\
\hline 14 & $\begin{array}{l}1-7,15,17 \\
19,21,23,25 \\
(1,6,0,60)\end{array}$ & 108 & $\begin{array}{l}\text { AII } \\
3.60 \\
\text { LSFIT }\end{array}$ & $\begin{array}{l}56 \\
37 \\
54\end{array}$ & $\begin{array}{r}18.53 \\
3.21 \\
1.36\end{array}$ & $\begin{array}{l}0.9830 \\
0.9830 \\
0.9772\end{array}$ & $\begin{array}{l}18.411 \\
18.194 \\
18.279\end{array}$ & $\begin{array}{l}0.523 \\
0.420 \\
0.137\end{array}$ & $\begin{array}{l}1.003 \\
0.954 \\
0.872\end{array}$ \\
\hline 15 & $\begin{array}{l}1,9-13,15 \\
17,19,21,23,25 \\
(1,0,5,60)\end{array}$ & 109 & $\begin{array}{l}\text { AII } \\
3.6 \sigma \\
\text { LSFIT }\end{array}$ & $\begin{array}{l}53 \\
43 \\
53\end{array}$ & $\begin{array}{l}8.24 \\
3.72 \\
1.41\end{array}$ & $\begin{array}{l}0.9852 \\
0.9852 \\
0.9805\end{array}$ & $\begin{array}{l}18.629 \\
18.535 \\
18.625\end{array}$ & $\begin{array}{l}0.311 \\
0.452 \\
0.131\end{array}$ & $\begin{array}{l}0.911 \\
0.968 \\
0.875\end{array}$ \\
\hline 16 & $\begin{array}{l}1,8-13,15, \\
17,19,21,23,25 \\
(1,0,6,60)\end{array}$ & 110 & $\begin{array}{l}\text { A1I } \\
3.6 \sigma \\
\text { LSFIT }\end{array}$ & $\begin{array}{l}53 \\
38 \\
53\end{array}$ & $\begin{array}{r}10.53 \\
2.90 \\
1.21\end{array}$ & $\begin{array}{l}0.9973 \\
0.9973 \\
0.9964\end{array}$ & $\begin{array}{l}20.400 \\
20.180 \\
20.490\end{array}$ & $\begin{array}{l}0.337 \\
0.353 \\
0.113\end{array}$ & $\begin{array}{l}0.920 \\
0.926 \\
0.864\end{array}$ \\
\hline
\end{tabular}




\begin{tabular}{|c|c|c|c|c|c|}
\hline 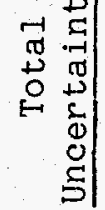 & 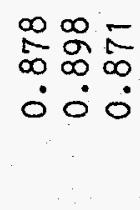 & 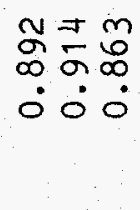 & 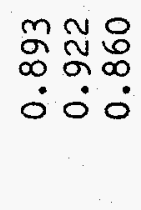 & 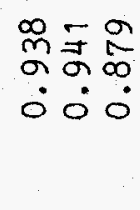 & 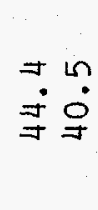 \\
\hline 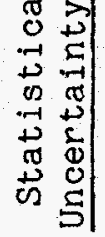 & 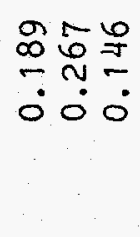 & 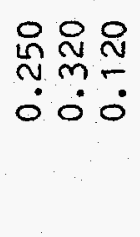 & 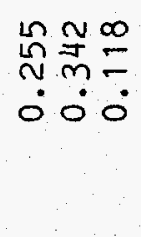 & 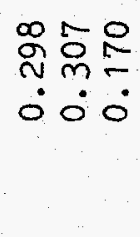 & $\begin{array}{l}m=7 \\
\infty \\
\infty \\
\end{array}$ \\
\hline 至| & 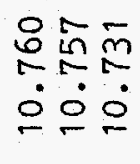 & $\begin{array}{l}\sigma \omega N \\
\exists m \equiv \\
\approx \sim N\end{array}$ & 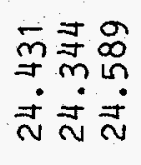 & $\begin{array}{l}0 \text { 요 } \\
\overline{0} \mathrm{n} \\
\dot{0} \dot{0} \\
\dot{N} 0\end{array}$ & $\begin{array}{l}0.5 \\
0 \% \\
0.0 \\
00\end{array}$ \\
\hline 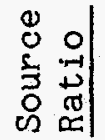 & 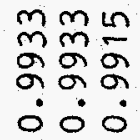 & 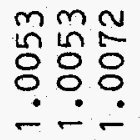 & 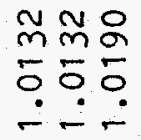 & $\begin{array}{lll}0 & 0 & = \\
0 & 0 & \text { In } \\
0 & 0 & 0 \\
0 & 0 & 0\end{array}$ & $\begin{array}{l}\circ 8 \\
80 \\
80 \\
\circ \\
-\div\end{array}$ \\
\hline & $\begin{array}{l}\stackrel{m}{m} \stackrel{m}{m} \\
\dot{m} \dot{\sim}\end{array}$ & $\begin{array}{l}\text { mo } \\
\text { 뭉 }\end{array}$ & 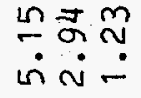 & 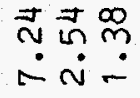 & $\begin{array}{l} \pm 0 \\
\dot{0}:\end{array}$ \\
\hline $\begin{array}{ll}c & 0 \\
0 & 0 \\
\vdots & 0 \\
\vdots & 0\end{array}$ & ถูก & 늠요 & แก กั & 吅 & $\vec{b} \hat{~}$ \\
\hline $\begin{array}{l}0 \\
0 \\
\vdots \\
\vdots \\
\Sigma\end{array}$ & 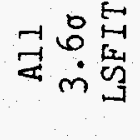 & 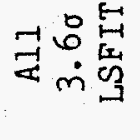 & 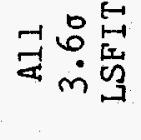 & 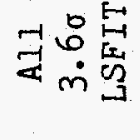 & $\begin{array}{l}-1 \\
0 \\
\dot{1}\end{array}$ \\
\hline 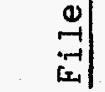 & $\Xi$ & $\stackrel{N}{=}$ & $\frac{m}{=}$ & $\Xi$ & $\stackrel{10}{=}$ \\
\hline 品 & 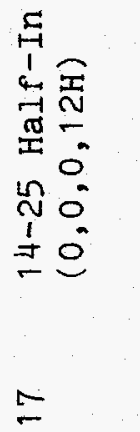 & 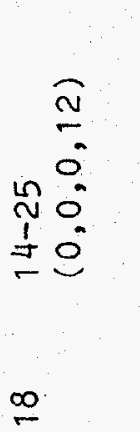 & 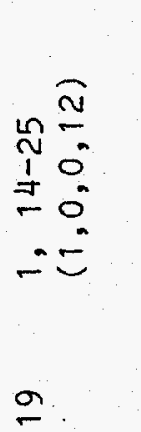 & 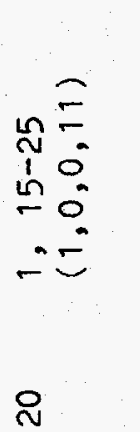 & 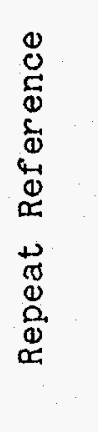 \\
\hline
\end{tabular}


TABLE 2.3 Worths of Control Rod Banks in ZPPR-17B

\begin{tabular}{|c|c|c|c|c|c|c|c|}
\hline$\underline{S t e p}^{a}$ & Control Rods ${ }^{\mathrm{b}}$ & $\begin{array}{c}\text { Experimental } \\
\text { Worth, } \$ \\
\end{array}$ & $\begin{array}{c}\text { Statistical } \\
10, \% \\
\end{array}$ & $\begin{array}{c}\text { Correlated } \\
10, \% \\
\end{array}$ & $\begin{array}{c}\text { Calculated } \\
k-e f f^{c} \\
\end{array}$ & $\begin{array}{l}\text { Calculated } \\
\text { Worth, } \$ \\
\end{array}$ & $C / E$ \\
\hline 1 & $\begin{array}{l}\text { Central CR } \\
(1,0,0,0)\end{array}$ & 1.001 & 0.087 & 0.811 & 0.982117 & 0.955 & 0.954 \\
\hline 3 & $\begin{array}{l}\text { IR } \\
(0,6,0,0)\end{array}$ & 5.822 & 0.116 & 0.866 & 0.966887 & 5.708 & 0.980 \\
\hline 2 & $\begin{array}{l}\text { IR Half Inserted } \\
(0,6 \mathrm{H}, 0,0)\end{array}$ & 3.171 & 0.123 & 0.865 & 0.975199 & 3.095 & 0.976 \\
\hline 10 & $\begin{array}{l}M R \\
(0,0,6,0)\end{array}$ & 8.318 & 0.121 & 0.859 & 0.959562 & 8.048 & 0.968 \\
\hline 18 & $\begin{array}{l}\mathrm{OR} \\
(0,0,0,12)\end{array}$ & 22.477 & 0.120 & 0.855 & 0.917563 & 22.186 & 0.987 \\
\hline 17 & $\begin{array}{l}\text { OR Half Inserted } \\
(0,0,0,12 \mathrm{H})\end{array}$ & 10.731 & 0.146 & 0.859 & 0.951408 & 10.695 & 0.997 \\
\hline
\end{tabular}

$a_{\text {Refer to Table } 2.2 .}$

${ }^{b_{I R}}=$ inner $r$ ing, $M R=$ middle ring, $O R=$ outer $r i n g, H=$ half-inserted.

Finite difference diffusion, xyz geometry, 6 energy groups, reference k-effective 0.985234 .

$a_{B}$-effective $=0.3374 \%$. 
TABLE 2.4 Worths of Rod Bank Combinations in ZPPR-17B

\begin{tabular}{|c|c|c|c|c|c|c|c|}
\hline Step $^{a}$ & Control Rods ${ }^{b}$ & $\begin{array}{l}\text { Experimental } \\
\text { Worth, } \$ \\
\end{array}$ & $\begin{array}{c}\text { Statistical } \\
10, \% \\
\end{array}$ & $\begin{array}{c}\text { Correlated } \\
10, \% \\
\end{array}$ & $\begin{array}{c}\text { Calculated } \\
\mathrm{k}-\mathrm{eff}^{\mathrm{c}} \\
\end{array}$ & $\begin{array}{l}\text { Calculated } \\
\text { Worth, } \$ d \\
\end{array}$ & $\mathrm{C} / \mathrm{E}$ \\
\hline 4 & $\begin{array}{l}\text { Center + IR } \\
(1,6,0,0)\end{array}$ & 6.137 & 0.117 & 0.869 & 0.965778 & 6.060 & 0.987 \\
\hline 9 & $\begin{array}{l}\text { Center }+ \text { MR } \\
(1,0,6,0)\end{array}$ & 9.017 & 0.126 & 0.861 & 0.957436 & 8.734 & 0.968 \\
\hline 19. & $\begin{array}{l}\text { Center }+ \text { OR } \\
(1,0,0,12)\end{array}$ & 24.589 & 0.118 & 0.852 & 0.912298 & 24.050 & 0.978 \\
\hline 6 & $\begin{array}{l}\mathrm{IR}+\mathrm{MR} \\
(0,6,6,0)\end{array}$ & 11.952 & 0.163 & 0.870 & 0.948020 & 11.809 & 0.988 \\
\hline 13 & $\begin{array}{l}\text { IR }+ \text { OR odd } \\
(0,6,0,6 \emptyset)\end{array}$ & 17.856 & 0.147 & 0.858 & 0.931124 & 17.482 & 0.979 \\
\hline 5 & $\begin{array}{l}\text { Center }+\mathrm{IR}+\mathrm{MR} \\
(1,6,6,0)\end{array}$ & 12.169 & 0.158 & 0.874 & 0.947343 & 12.032 & 0.989 \\
\hline 14 & $\begin{array}{l}\text { Center + IR } \\
\quad+\text { OR odd } \\
(1,6,0,60)\end{array}$ & 18.279 & 0.137 & 0.861 & 0.929798 & 17.936 & 0.981 \\
\hline 16 & $\begin{array}{c}\text { Center + MR } \\
+ \text { OR odd } \\
(1,0,6,6 \emptyset)\end{array}$ & 20.490 & 0.113 & 0.857 & 0.923764 & 20.018 & 0.977 \\
\hline
\end{tabular}

a Refer to Table 2.2.

${ }^{b} I R=$ inner $r$ ing, $M R=$ middle $r i n g, O R=$ outer ring, $\varnothing=$ odd numbered rods .

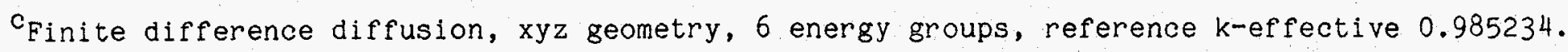

$d_{\beta}$-effective $=0.3374 \%$. 
TABLE 2.5 Worths of Rod Banks with Missing Rods in ZPPR-17B

\begin{tabular}{|c|c|c|c|c|c|c|c|}
\hline Step $^{a}$ & Control Rods ${ }^{b}$ & $\begin{array}{c}\text { Experimental } \\
\text { Worth, } \$ \\
\end{array}$ & $\begin{array}{c}\text { Statistical } \\
10, \% \\
\end{array}$ & $\begin{array}{c}\text { Correlated } \\
10, \% \\
\end{array}$ & $\begin{array}{c}\text { Calculated } \\
\text { k-eff } \\
\end{array}$ & $\begin{array}{l}\text { Calculated } \\
\text { Worth, } \$ d \\
\end{array}$ & $\mathrm{C} / \mathrm{E}$ \\
\hline 11 & $\begin{array}{l}\text { MR-CR8 } \\
(0,0,5,0)\end{array}$ & 6.537 & 0.134 & 0.863 & 0.964752 & 6.387 & 0.977 \\
\hline 8 & $\begin{array}{l}\text { Center, IR-CR2, MR } \\
(1,5,6,0)\end{array}$ & 11.718 & 0.161 & 0.872 & 0.948817 & 11.546 & 0.985 \\
\hline 12 & $\begin{array}{l}\text { Center, IR-CR2, } \\
\text { OR odd } \\
(1,5,0,60)\end{array}$ & $17 \cdot 321$ & 0.131 & 0.860 & 0.932462 & 17.025 & 0.983 \\
\hline 7 & $\begin{array}{l}\text { Center, IR, MR-CR8 } \\
(1,6,5,0)\end{array}$ & 10.804 & 0.180 & 0.880 & 0.951187 & 10.768 & 0.997 \\
\hline 15 & $\begin{array}{l}\text { Center, MR-CR8, } \\
\text { OR odd } \\
(1,0,5,60)\end{array}$ & 18.625 & 0.161 & 0.860 & 0.928746 & 18.297 & 0.982 \\
\hline 20 & $\begin{array}{l}\text { Center, OR-CR14 } \\
(1,0,0,11)\end{array}$ & 20.435 & 0.170 & 0.862 & 0.922999 & 20.284 & 0.993 \\
\hline
\end{tabular}

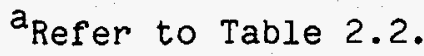

$b_{\text {IR }}=$ inner ring, $M R=$ middle ring, $O R=$ outer ring, $\emptyset=$ odd numbered rods .

CFinite difference, xyz geometry, 6 energy groups, reference k-effective 0.985234 .

$\mathrm{d}_{\text {Beffective }}=0.3374 \%$. 
TABLE 2.6 Control Rod Interaction Effects in ZPPR-17B

\begin{tabular}{|c|c|c|c|c|c|c|}
\hline $\begin{array}{l}\text { Control } \\
\text { Rods } \\
\end{array}$ & $\begin{array}{l}\text { From } \\
\text { Steps }\end{array}$ & $\begin{array}{c}\text { Rods Inserted } \\
\text { in Core } \\
\end{array}$ & $\begin{array}{c}\text { Exper imental } \\
\text { Worth, } \$\end{array}$ & $\begin{array}{c}\text { Statistical } \\
\text { Uncertainty, \% }\end{array}$ & $\begin{array}{l}\text { Calculated } \\
\text { Worth, } \$ \\
\end{array}$ & $\mathrm{C} / \mathrm{E}$ \\
\hline$C R$ & $\begin{array}{c}1 \\
6,5 \\
4,3 \\
14,13 \\
9,10 \\
19,18\end{array}$ & $\begin{array}{l}\text { None } \\
\text { IR + MR } \\
\text { IR } \\
\text { IR + 60R odd } \\
\text { MR } \\
\text { OR }\end{array}$ & $\begin{array}{l}1.001 \\
0.217 \\
0.315 \\
0.423 \\
0.699 \\
2.112\end{array}$ & $\begin{array}{r}0.1 \\
12.6 \\
3.1 \\
8.6 \\
2.2 \\
1.7\end{array}$ & $\begin{array}{l}0.955 \\
0.223 \\
0.352 \\
0.454 \\
0.686 \\
1.864\end{array}$ & $\begin{array}{l}0.954 \\
1.028 \\
1.117 \\
1.073 \\
0.981 \\
0.883\end{array}$ \\
\hline IR & $\begin{array}{c}3 \\
6,10\end{array}$ & $\begin{array}{l}\text { None } \\
\text { MR }\end{array}$ & $\begin{array}{l}5.823 \\
3.634\end{array}$ & $\begin{array}{l}0.1 \\
0.6\end{array}$ & $\begin{array}{l}5.708 \\
3.761\end{array}$ & $\begin{array}{l}0.980 \\
1.035\end{array}$ \\
\hline MR & $\begin{array}{l}10 \\
6,3\end{array}$ & $\begin{array}{l}\text { None } \\
\text { IR }\end{array}$ & $\begin{array}{l}8.318 \\
6.130\end{array}$ & $\begin{array}{l}0.1 \\
0.3\end{array}$ & $\begin{array}{l}8.048 \\
6.101\end{array}$ & $\begin{array}{l}0.968 \\
0.995\end{array}$ \\
\hline 6 OR odd & $\begin{array}{l}13,3 \\
14,4 \\
16,9\end{array}$ & $\begin{array}{l}I R \\
\text { Center + IR } \\
\text { Center + MR }\end{array}$ & $\begin{array}{l}12.034 \\
12.142 \\
11.475\end{array}$ & $\begin{array}{l}0.2 \\
0.2 \\
0.2\end{array}$ & $\begin{array}{l}11.774 \\
11.876 \\
11.284\end{array}$ & $\begin{array}{l}0.978 \\
0.978 \\
0.983\end{array}$ \\
\hline
\end{tabular}


TABLE 2.7 Worth of Single Rods Withdrawn from Rod Banks

\begin{tabular}{|c|c|c|c|c|c|c|}
\hline $\begin{array}{l}\text { Control } \\
\text { Rods } \\
\end{array}$ & $\begin{array}{l}\text { From } \\
\text { Steps }\end{array}$ & $\begin{array}{l}\text { Rods Inserted } \\
\text { in Core } \\
\end{array}$ & $\begin{array}{c}\text { Exper imental } \\
\text { Worth, } \$ \\
\end{array}$ & $\begin{array}{c}\text { Statistical } \\
\text { Uncertainty, } \%\end{array}$ & $\begin{array}{c}\text { Calculated } \\
\text { Worth, } \$\end{array}$ & $\mathrm{C} / \mathrm{E}$ \\
\hline \multirow[t]{2}{*}{ CR2 } & 5,8 & $\begin{array}{l}\text { Center }+6 I R \\
+6 M R\end{array}$ & 0.451 & 6.0 & 0.486 & 1.078 \\
\hline & 14,12 & $\begin{array}{l}\text { Center }+6 I R \\
+60 R \text { odd }\end{array}$ & 0.958 & 3.5 & 0.911 & 0.970 \\
\hline $\begin{array}{l}\text { Mean Worth } \\
\text { in Bank }\end{array}$ & 3 & $6 \mathrm{IR}$ & 0.970 & 0.1 & 0.951 & 0.980 \\
\hline \multirow[t]{2}{*}{ CR8 } & $\begin{array}{r}10,11 \\
5,7\end{array}$ & $\begin{array}{l}6 M R \\
\text { Center + 6IR } \\
+6 M R\end{array}$ & $\begin{array}{l}1.781 \\
1.365\end{array}$ & $\begin{array}{l}0.7 \\
2.0\end{array}$ & $\begin{array}{l}1.661 \\
1.264\end{array}$ & $\begin{array}{l}0.933 \\
0.926\end{array}$ \\
\hline & 16,15 & $\begin{array}{l}\text { Center + 6MR } \\
+60 R \text { odd }\end{array}$ & 1.865 & 2.0 & 1.721 & 0.923 \\
\hline $\begin{array}{l}\text { Mean Worth } \\
\text { in Bank }\end{array}$ & 10 & $6 \mathrm{MR}$ & 1.386 & 0.1 & 1.341 & 0.968 \\
\hline CR14 & 19,20 & Center $+120 R$ & 4.154 & 1.1 & 3.766 & 0.907 \\
\hline $\begin{array}{l}\text { Mean Worth } \\
\text { in Bank }\end{array}$ & 18 & $120 \mathrm{R}$ & 1.873 & 0.1 & 1.849 & 0.987 \\
\hline
\end{tabular}


TABLE 2.8 Calculated Control Rod Worths in ZPPR-17B using Nodal Diffusion and Nodal Transport Models

\begin{tabular}{|c|c|c|c|c|c|c|}
\hline Control Rods & $\begin{array}{c}\text { FDDT } \\
\text { Worth, } \$\end{array}$ & $\begin{array}{c}\text { NDT } \\
\text { Worth, } \$ \\
\end{array}$ & $\begin{array}{l}\text { Mesh } \\
\text { Correction, } \% \\
\end{array}$ & $\begin{array}{c}\text { NTT } \\
\text { Worth, } \$ \\
\end{array}$ & $\begin{array}{c}\text { Transport } \\
\text { Correction, } \$\end{array}$ & $\mathrm{C} / \mathrm{E}$ \\
\hline CR1 & 0.955 & 1.044 & +9.3 & 1.042 & -0.2 & 1.041 \\
\hline IR & 5.708 & 6.211 & +8.8 & 6.136 & -1.2 & 1.054 \\
\hline MR & 8.048 & 8.918 & +10.8 & 8.694 & -2.5 & 1.045 \\
\hline$\emptyset \mathrm{R}$ & 22.186 & 24.776 & +11.7 & 23.643 & -4.8 & 1.052 \\
\hline $\mathrm{CR} 1+\mathrm{IR}$ & 6.060 & 6.555 & +8.2 & 6.488 & -1.0 & 1.057 \\
\hline $\mathrm{CR} 1+\mathrm{MR}$ & 8.734 & 9.635 & +10.3 & 9.420 & -2.2 & 1.045 \\
\hline $\mathrm{CR} 1+\emptyset \mathrm{R}$ & 24.050 & 26.966 & +12.1 & 25.796 & -4.3 & 1.049 \\
\hline$I R+M R$ & 11.809 & 12.805 & +8.4 & 12.582 & -1.8 & 1.053 \\
\hline $\mathrm{CR} 1+\mathrm{IR}+\mathrm{MR}$ & 12.032 & 13.011 & +8.1 & 12.797 & -1.7 & 1.052 \\
\hline
\end{tabular}




\section{MEASUREMENT AND CALCULATION OF CONTROL ROD WORTHS IN ZPPR-17C \\ (T. Sanda,* P. J. Collins, D. M. Smith and G. L. Grasseschi) \\ 3.1 Introduction}

The ZPPR-17C reference core simulated a beginning-of-cycle loading with primary control rods -- center and 12 outer ring - half inserted. The control rod worth measurements provide data on the worths of full insertion of secondary control banks in this loading. The secondary rod locations were the six inner ring (IR) positions and the six middle ring (MR) positions. The basic rod banks were six inner ring (6IR), six middle ring (6MR), 3IR, 3MR and a combination 3IR + 3MR. The three-rod patterns were measured both for the rods symmetrically disposed around the hexagonal bank and for the three rods in adjacent locations. Several cases of banks with missing rods were also measured, $5 I R, 5 M R, 3 I R+2 M R$ and $2 I R+3 M R$.

Three of the rod patterns, 6IR, 6MR and 5MR, were also measured in ZPPR-17B, the end-of-cycle simulation with all rods withdrawn. These cases provide a comparison of the interaction effects and of the accuracy of calculation of rod worths in an operating condition rather than in a reference core with no rods inserted.

\subsection{Description of the Measurements}

The measurements were made in the $Z P P R-17 \mathrm{C}$ subcritical reference loading described in ANL-ZPR-481 p. 4. The control rod locations, shown in Fig. 3.1, were the same as those in $Z P P R-17 B$. The measurement techniques followed those used for ZPPR-17B. The mockup control rods occupied four matrix positions with the drawers fully loaded with natural $B_{4} C$ plates. The drawer master was 17-0-603. Compositions are given in Table 2.1, in the previous section.

\footnotetext{
*On assignment from Power Reactor and Nuclear Fuel Development Corporation
} (PNC). 
The measurements were made between June 12, 1987 and June 19, 1987, in reactor loadings 135 to 148 , reactor runs 150 to 165 . The data were recorded on files 136 to 149 of the ZPPR-17 64-detector datafile. The reference reactivity for the series was measured by inverse-kinetics analysis of a rod drop in reactor loadings 135, run 150. The 64-detector. data for this reference were taken in run 150 and recorded on file 136. The reference loading was restored immediately after the rod measurements in run 165 and the data were recorded on file 149.

\subsection{Description of the Calculations}

Calculations of detector efficiencies and effective source ratios were made with an $x y z$ model and 6 group data as was done for ZPPR-17B (Section 2.3). The 6 group data used the microscopic cross sections produced for 17B and were not separately collapsed for 17C. The calculation model for $Z P P R-17 \mathrm{C}$ is given in ANL-ZPR-481, p. 11 .

Only two of the control configurations permitted quarter-xy symmetry to be used, six cases required full-xy representation while two cases were symmetric about the $y$-axis and one was symmetric about the $x-$ axis. All configurations were calculated with the full-xy model to avoid proliferation of models.

Since the control rod worths were measured with the rods fully inserted, the $x y$ models used half $-z$ symmetry. Shielding factors for the half-inserted rods in the reference core, 0.292 for the CRs and 0.708 for the CRPs, were used to adjust the k-effective in this model to 0.9888 . The $k$-effective for the reference core using a quarter-zy full-z model was 0.989323 . The two control patterns with quarter-y symmetry ( 6 inner ring and 6 middle $r i n g$ ) were also calculated with the full-z model to check the results from the approximate models.

Calculations of detector efficiencies and effective source worths were made with the finite-difference diffusion path of DIF3D. Calculated worths for the two symmetric rod banks were also obtained with the nodal diffusion and nodal transport solutions. 


\subsection{Details of the Experimental Data Processing}

A summary of the data processing using the MCCRUNCH code is given in Table 3.1. The abbreviations $\mathrm{nIR}$, nMR are used for the $\mathrm{n}$ rods in the inner $r$ ing and the middle ring. Detectors which were immediately adjacent to any of the inserted rods were omitted from the analysis.

The two configurations processed with both half-z and full-z models are shown as steps $7 \mathrm{~A}$ and $7 \mathrm{~B}$ (6IR) and steps $11 \mathrm{~A}$ and $11 \mathrm{~B}$ (6MR). These cases show that the efficiencies calculated with the half-z model give satisfactory results. For the LSFIT method, the differences in experimental worths were only $0.02 \%(6 I R)$ and $0.06 \%$ (6MR).

The recommended control rod worths are taken from the LSFIT analysis. These values are summarized and compared with calculation in the next section.

\subsection{Recommended Worths and Comparisons with Calculation}

The recommended worths are given in Table 3.2. Statistical and correlated uncertainty components are given to three figures to facilitate derivation of uncertainties when subtracting configuration worths. The calculated worths in Table 3.3 are obtained from the half-z model.

The C/E results vary by only $3 \%$ over the range of the configurations. The highest values are for the three adjacent rods in the inner and middle ring. In both cases, these are predicted $2 \%$ higher than their counterparts which have three rods symmetrically disposed around the ring. Apart from these two cases, the $C / E$ values are in the range 0.949 to 0.963 .

Rod interaction effects are shown in Table 3.3 in terms of the worth per rod (WPR) in banks of $6,5,3$ and 2 rods. The inner ring rods show a negative interaction (WPR for the 3 symmetric rods is 15\% higher than for the bank of 6 rods or 3 adjacent rods). The wider-spaced rods in the middle ring show a more neutral interaction (WPR for the 3 symmetric rods is 
the same as that for the bank of 6 rods, but 25\% greater than for 3 adjacent rods). The worth of either bank of 3 rods is suppresed by $20 \%$ when the other bank is also present. These latter effects are predicted with a C/E bias similar to that for the individual banks.

Improved calculations have been made for the complete inner and middle banks of rods which were symmetric in the $x y$-plane. These calculations used full-z representation to model the half-inserted rods in the reference loading. Calculations were made with finite-difference diffusion (FDDT), with nodal diffusion (NDT) which has a small mesh-size error and with nodal transport (NTT). The calculated rod bank worths are compared in Table 3.4 .

The effect in k-effective due to using the half-z model vary a little between the reference and the control bank cases and result in changes in rod worths of $0.5 \%$ and $0.7 \%$. Corrections to rod worths for diffusion theory mesh size are $+10 \%$ and $+11 \%$ while transport corrections are $-0.6 \%$ and $-2 \%$. The mesh corrections are considerably larger than found in other cores. Similar mesh and transport corrections were found for ZPPR17B. Thus the effects are due to the internal blanket rather than the rods which were half-inserted in the core.

The worths of the inner bank, the middle bank and five rods in the middle bank were measured both in ZPPR-17B and in ZPPR-17C. The experimental worths and predictions are compared in Table 3.5. The worth of the inner bank is similar in either core but the worth of the middle bank is depressed by $8 \%$ in $17 \mathrm{C}$ due to the half-inserted outer bank. The $\mathrm{C} / \mathrm{E}$ results are consistent between $17 \mathrm{~B}$ and $17 \mathrm{C}$ within the (total) uncertainties of the experiments of about $0.9 \%$. 


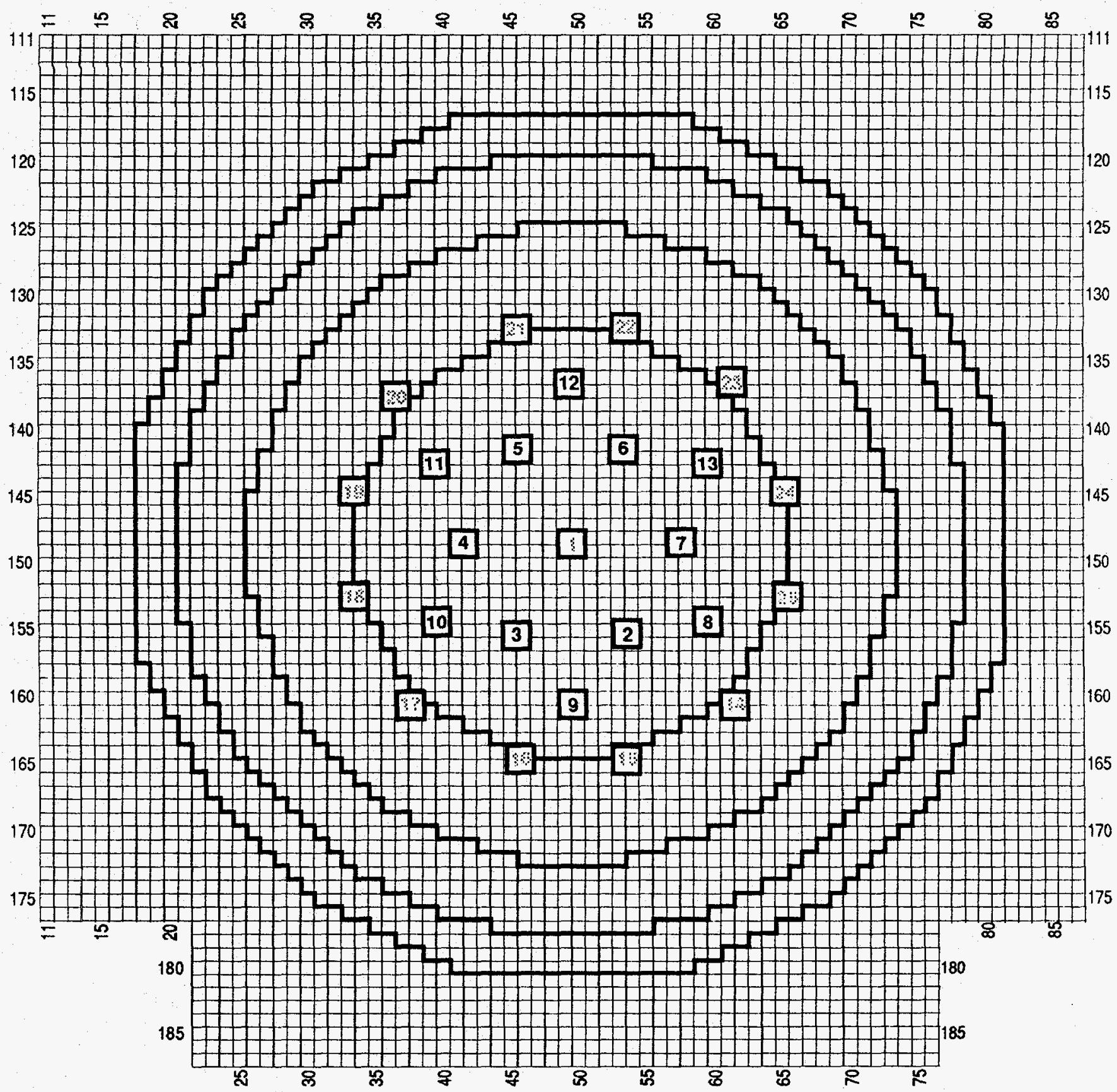

Half - Inserted Control Rod

Fig. 3.1. Control Rod Locations in ZPPR-17C 
TABLE 3.1 Data Processing for Control Rod Worth Measurements in ZPPR-17C

\begin{tabular}{|c|c|c|c|c|c|c|c|c|c|}
\hline Step & Control Rods & File & Method & $\begin{array}{l}\text { No. of } \\
\text { FCs } \\
\end{array}$ & $x^{2}$ & $\begin{array}{l}\text { Source } \\
\text { Ratio } \\
\end{array}$ & Worth, \$ & $\begin{array}{c}\text { Statistical } \\
\text { Uncertainty, \% }\end{array}$ & $\begin{array}{c}\text { Total } \\
\text { Uncertainty, } \$ \\
\end{array}$ \\
\hline 1 & $\begin{array}{l}3,5,7 \\
3 \mathrm{IR}\end{array}$ & 138 & $\begin{array}{c}\text { AlI } \\
3.6 \sigma \\
\text { LSFIT }\end{array}$ & $\begin{array}{l}62 \\
55 \\
62\end{array}$ & $\begin{array}{r}12.06 \\
3.51 \\
1.23\end{array}$ & $\begin{array}{l}0.9948 \\
0.9948 \\
0.9930\end{array}$ & $\begin{array}{l}3.388 \\
3.367 \\
3.390\end{array}$ & $\begin{array}{l}0.293 \\
0.299 \\
0.111\end{array}$ & $\begin{array}{l}0.915 \\
0.917 \\
0.875\end{array}$ \\
\hline 2 & $\begin{array}{l}3,5,7,11,13 \\
3 I R+2 M R\end{array}$ & 139 & $\begin{array}{l}\text { All } \\
3.6 \sigma \\
\text { LSFIT }\end{array}$ & $\begin{array}{l}61 \\
51 \\
59\end{array}$ & $\begin{array}{r}14.56 \\
3.35 \\
1.35\end{array}$ & $\begin{array}{l}0.9786 \\
0.9786 \\
0.9735\end{array}$ & $\begin{array}{l}5.398 \\
5.363 \\
5.370\end{array}$ & $\begin{array}{l}0.439 \\
0.360 \\
0.168\end{array}$ & $\begin{array}{l}0.969 \\
0.936 \\
0.884\end{array}$ \\
\hline 3 & $\begin{array}{l}3,5,7,9,11,13 \\
3 I R+3 M R\end{array}$ & 140 & $\begin{array}{c}\text { All } \\
3.6 \sigma \\
\text { LSFIT }\end{array}$ & $\begin{array}{l}60 \\
42 \\
55\end{array}$ & $\begin{array}{r}17.65 \\
3.89 \\
1.40\end{array}$ & $\begin{array}{l}0.9861 \\
0.9861 \\
0.9826\end{array}$ & $\begin{array}{l}6.612 \\
6.574 \\
6.613\end{array}$ & $\begin{array}{l}0.418 \\
0.411 \\
0.187\end{array}$ & $\begin{array}{l}0.959 \\
0.955 \\
0.885\end{array}$ \\
\hline 4 & $\begin{array}{l}5,7,9,11,13 \\
2 I R+3 M R\end{array}$ & 141 & $\begin{array}{c}\text { A11 } \\
3.6 \sigma \\
\text { LSFIT }\end{array}$ & $\begin{array}{l}61 \\
50 \\
60\end{array}$ & $\begin{array}{r}12.59 \\
3.48 \\
1.39\end{array}$ & $\begin{array}{l}0.9862 \\
0.9862 \\
0.9828\end{array}$ & $\begin{array}{l}5.658 \\
5.637 \\
5.636\end{array}$ & $\begin{array}{l}0.400 \\
0.389 \\
0.192\end{array}$ & $\begin{array}{l}0.951 \\
0.947 \\
0.887\end{array}$ \\
\hline 5 & $\begin{array}{l}5,6,7 \\
3 \mathrm{IR}\end{array}$ & 142 & $\begin{array}{l}\text { All } \\
3.60 \\
\text { LSFIT }\end{array}$ & $\begin{array}{l}63 \\
49 \\
62\end{array}$ & $\begin{array}{r}21.74 \\
3.12 \\
1.41\end{array}$ & $\begin{array}{l}0.9735 \\
0.9735 \\
0.9687\end{array}$ & $\begin{array}{l}2.876 \\
2.856 \\
2.850\end{array}$ & $\begin{array}{l}0.371 \\
0.259 \\
0.138\end{array}$ & $\begin{array}{l}0.944 \\
0.906 \\
0.883\end{array}$ \\
\hline 6 & $\begin{array}{l}3,4,5,6,7 \\
5 \mathrm{IR}\end{array}$ & 143 & $\begin{array}{c}\text { AlI } \\
3.6 \sigma \\
\text { LSFIT }\end{array}$ & $\begin{array}{l}62 \\
45 \\
58\end{array}$ & $\begin{array}{r}17.68 \\
3.84 \\
1.40\end{array}$ & $\begin{array}{l}0.9812 \\
0.9812 \\
0.9751\end{array}$ & $\begin{array}{l}5.055 \\
5.022 \\
5.039\end{array}$ & $\begin{array}{l}0.442 \\
0.405 \\
0.187\end{array}$ & $\begin{array}{l}0.970 \\
0.954 \\
0.889\end{array}$ \\
\hline $7 A^{a}$ & $\begin{array}{l}2,3,4,5,6,7 \\
6 \mathrm{IR}\end{array}$ & 144 & $\begin{array}{c}\text { AlI } \\
3.60 \\
\text { LSFIT }\end{array}$ & $\begin{array}{l}62 \\
43 \\
59\end{array}$ & $\begin{array}{r}19.47 \\
3.10 \\
1.37\end{array}$ & $\begin{array}{l}0.9794 \\
0.9794 \\
0.9731\end{array}$ & $\begin{array}{l}5.926 \\
5.867 \\
5.884\end{array}$ & $\begin{array}{l}0.507 \\
0.360 \\
0.201\end{array}$ & $\begin{array}{l}1.001 \\
0.935 \\
0.891\end{array}$ \\
\hline $7 B^{a}$ & $\begin{array}{l}2,3,4,5,6,7 \\
6 \mathrm{IR}\end{array}$ & 144 & $\begin{array}{l}\text { AII } \\
3.60 \\
\text { LSEIT }\end{array}$ & $\begin{array}{l}62 \\
42 \\
62\end{array}$ & $\begin{array}{r}22.86 \\
2.86 \\
1.27\end{array}$ & $\begin{array}{l}0.9813 \\
0.9813 \\
0.9730\end{array}$ & $\begin{array}{l}5.910 \\
5.821 \\
5.869\end{array}$ & $\begin{array}{l}0.532 \\
0.332 \\
0.132\end{array}$ & $\begin{array}{l}1.014 \\
0.925 \\
0.880\end{array}$ \\
\hline 8 & $\begin{array}{l}11,12,13 \\
3 \mathrm{MR}\end{array}$ & 145 & $\begin{array}{l}\text { AII } \\
3.60 \\
\text { LSFIT }\end{array}$ & $\begin{array}{l}62 \\
40 \\
59\end{array}$ & $\begin{array}{r}17.57 \\
2.79 \\
1.37\end{array}$ & $\begin{array}{l}0.9550 \\
0.9550 \\
0.9475\end{array}$ & $\begin{array}{l}3.081 \\
3.068 \\
3.052\end{array}$ & $\begin{array}{l}0.331 \\
0.285 \\
0.123\end{array}$ & $\begin{array}{l}0.997 \\
0.982 \\
0.882\end{array}$ \\
\hline
\end{tabular}


TABLE 3.1 (contd)

\begin{tabular}{|c|c|c|c|c|c|c|c|c|c|}
\hline Step & Control Rods & File & Method & $\begin{array}{l}\text { No. of } \\
\text { FCs } \\
\end{array}$ & $x^{2}$ & $\begin{array}{l}\text { Source } \\
\text { Ratio } \\
\end{array}$ & Worth, \$ & $\begin{array}{l}\text { Statistical } \\
\text { Uncertainty, } \%\end{array}$ & $\begin{array}{c}\text { Total } \\
\text { Uncertainty, \$ } \\
\end{array}$ \\
\hline 9 & $\begin{array}{l}9,11,13 \\
\text { 3MR }\end{array}$ & 146 & $\begin{array}{c}\text { ALL } \\
3.6 \Sigma \\
\text { LSFIT }\end{array}$ & $\begin{array}{l}62 \\
56 \\
61\end{array}$ & $\begin{array}{l}9.05 \\
2.94 \\
1.18\end{array}$ & $\begin{array}{l}0.9984 \\
0.9984 \\
0.9978\end{array}$ & $\begin{array}{l}3.827 \\
3.809 \\
3.827\end{array}$ & $\begin{array}{l}0.256 \\
0.249 \\
0.099\end{array}$ & $\begin{array}{l}0.903 \\
0.901 \\
0.872\end{array}$ \\
\hline 10 & $\begin{array}{l}9,10,11,12,13 \\
5 \mathrm{MR}\end{array}$ & 147 & $\begin{array}{l}\text { Al1 } \\
3.60 \\
\text { LSFIT }\end{array}$ & $\begin{array}{l}60 \\
49 \\
59\end{array}$ & $\begin{array}{l}9.39 \\
4.57 \\
1.44\end{array}$ & $\begin{array}{l}0.9774 \\
0.9774 \\
0.9722\end{array}$ & $\begin{array}{l}6.058 \\
6.029 \\
6.045\end{array}$ & $\begin{array}{l}0.315 \\
0.530 \\
0.190\end{array}$ & $\begin{array}{l}0.920 \\
1.014 \\
0.889\end{array}$ \\
\hline $11 A^{a}$ & $\begin{array}{l}8,9,10,11,12,13 \\
6 \mathrm{MR}\end{array}$ & 148 & $\begin{array}{l}\text { All } \\
3.60 \\
\text { LSFIT }\end{array}$ & $\begin{array}{l}60 \\
39 \\
55\end{array}$ & $\begin{array}{r}12.28 \\
2.77 \\
1.43\end{array}$ & $\begin{array}{l}0.9946 \\
0.9946 \\
0.9932\end{array}$ & $\begin{array}{l}7.682 \\
7.698 \\
7.730\end{array}$ & $\begin{array}{l}0.349 \\
0.345 \\
0.211\end{array}$ & $\begin{array}{l}0.930 \\
0.928 \\
0.888\end{array}$ \\
\hline $11 \mathrm{~B}^{\mathrm{a}}$ & $\begin{array}{l}8,9,10,11,12,13 \\
6 \mathrm{MR}\end{array}$ & 148 & $\begin{array}{l}\text { Al1 } \\
3.60 \\
\text { LSFIT }\end{array}$ & $\begin{array}{l}60 \\
46 \\
60\end{array}$ & $\begin{array}{r}13.32 \\
4.06 \\
1.35\end{array}$ & $\begin{array}{l}0.9976 \\
0.9976 \\
0.9966\end{array}$ & $\begin{array}{l}7.657 \\
7.583 \\
7.682\end{array}$ & $\begin{array}{l}0.339 \\
0.427 \\
0.114\end{array}$ & $\begin{array}{l}0.926 \\
0.962 \\
0.870\end{array}$ \\
\hline & Repeat Reference & 149 & $\begin{array}{l}\text { Al1 } \\
3.60\end{array}$ & $\begin{array}{l}64 \\
64\end{array}$ & $\begin{array}{l}1.16 \\
1.16\end{array}$ & $\begin{array}{l}1.0000 \\
1.0000\end{array}$ & $\begin{array}{l}0.0011 \\
0.0011\end{array}$ & $\begin{array}{r}9.36 \\
10.07\end{array}$ & $\begin{array}{l}70.25 \\
70.35\end{array}$ \\
\hline
\end{tabular}

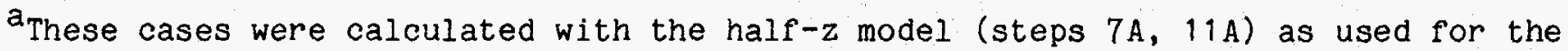
remaining cases and also with the full-z model (steps 7B, 11B) taking advantage of the quarter-xy symmetry.

$\mathrm{b}_{\mathrm{IR}}=$ inner ring, $M R=$ middle ring, OR $=$ outer ring. 
TABLE 3.2 Control Rod Worths in ZPPR-17C

\begin{tabular}{|c|c|c|c|c|c|c|c|}
\hline Step ${ }^{a}$ & $\begin{array}{l}\text { Control } \\
\text { Rods }\end{array}$ & $\begin{array}{c}\text { Experimental } \\
\text { Worth, } \$\end{array}$ & $\begin{array}{c}\text { Statistical } \\
10, \%\end{array}$ & $\begin{array}{c}\text { Correlated } \\
10, \%\end{array}$ & $\begin{array}{c}\text { Calculated } \\
k_{\text {eff }}\end{array}$ & $\begin{array}{l}\text { Calculated } \\
\text { Worth, } \$^{c}\end{array}$ & $C / E$ \\
\hline 7 & $6 \mathrm{IR}$ & 5.869 & 0.132 & 0.870 & 0.970615 & 5.651 & 0.963 \\
\hline 6 & $5 I R$ & 5.039 & 0.187 & 0.869 & 0.973270 & 4.814 & 0.955 \\
\hline 1 & $\begin{array}{l}3 \text { IR } \\
\text { symmetric }\end{array}$ & 3.390 & 0.111 & 0.868 & 0.978374 & 3.217 & 0.949 \\
\hline 5 & $\begin{array}{l}\text { 3IR } \\
\text { adjacent }\end{array}$ & 2.850 & 0.138 & 0.872 & 0.979829 & 2.765 & 0.970 \\
\hline 11 & $6 \mathrm{MR}$ & 7.682 & 0.114 & 0.862 & 0.965254 & 7.356 & 0.958 \\
\hline 10 & 5MR & 6.045 & 0.190 & 0.868 & 0.970106 & 5.812 & 0.963 \\
\hline 9 & $\begin{array}{l}\text { 3MR } \\
\text { symmetric }\end{array}$ & 3.827 & 0.099 & 0.866 & 0.976877 & 3.684 & 0.963 \\
\hline 8 & $\begin{array}{l}3 \mathrm{MR} \\
\text { adjacent }\end{array}$ & 3.052 & 0.123 & 0.873 & 0.979077 & 2.999 & 0.983 \\
\hline 3 & $3 I R+3 M R$ & 6.613 & 0.187 & 0.865 & 0.968448 & 6.338 & 0.958 \\
\hline 2 & $3 I R+2 M R$ & 5.370 & 0.168 & 0.868 & 0.972151 & 5.166 & 0.962 \\
\hline 4 & $2 I R+3 M R$ & 5.636 & 0.192 & 0.866 & 0.971377 & 5.411 & 0.960 \\
\hline
\end{tabular}

a Refer to Table 3.1. 6IR refers to 6 inner ring control rods etc.

${ }^{b}$ Calculations 6 group $x y z$ with half-z model, finite difference diffusion, reference $k=e f$ fective $=0.988823$.

CUsing Beffective $=0.3357 \%$. 
TABLE 3.3 Control Rod Interaction Effects in ZPPR-17C

\begin{tabular}{|c|c|c|c|c|}
\hline \multirow[b]{2}{*}{ Rod Bank } & \multicolumn{3}{|c|}{ Worth Per Rod } & \multirow[b]{2}{*}{$C / E$} \\
\hline & Measured, $\$$ & $\sigma_{S}, \not$ & Calculated, \$ & \\
\hline $6 I R$ & 0.978 & 0.13 & 0.942 & 0.963 \\
\hline $5 I R$ & 1.008 & 0.19 & 0.963 & 0.955 \\
\hline 3IR adjacent & 0.950 & 0.14 & 0.922 & 0.970 \\
\hline 3IR symmetric & 1.130 & 0.11 & 1.072 & 0.949 \\
\hline $\begin{array}{l}\text { 3IR symmetric with } \\
\text { 3MR inserted }\end{array}$ & 0.929 & 0.46 & 0.885 & 0.953 \\
\hline $\begin{array}{l}\text { 2IR with } \\
\text { 3MR inserted }\end{array}$ & 0.905 & 0.62 & 0.864 & 0.955 \\
\hline $6 \mathrm{MR}$ & 1.280 & 0.11 & 1.226 & 0.958 \\
\hline 5MR & 1.209 & 0.19 & 1.162 & 0.962 \\
\hline 3MR adjacent & 1.017 & 0.12 & 1.000 & 0.983 \\
\hline 3MR symmetric & 1.276 & 0.10 & 1.228 & 0.963 \\
\hline $\begin{array}{l}\text { 3MR symmetric with } \\
\text { 3IR inserted }\end{array}$ & 1.074 & 0.40 & 1.040 & 0.969 \\
\hline $\begin{array}{l}\text { 2MR with } \\
\text { 3IR inserted }\end{array}$ & 0.990 & 0.49 & 0.974 & 0.984 \\
\hline
\end{tabular}


TABLE 3.4 Comparison of Control Rod Worths in ZPPR-17C using Different Calculation Models

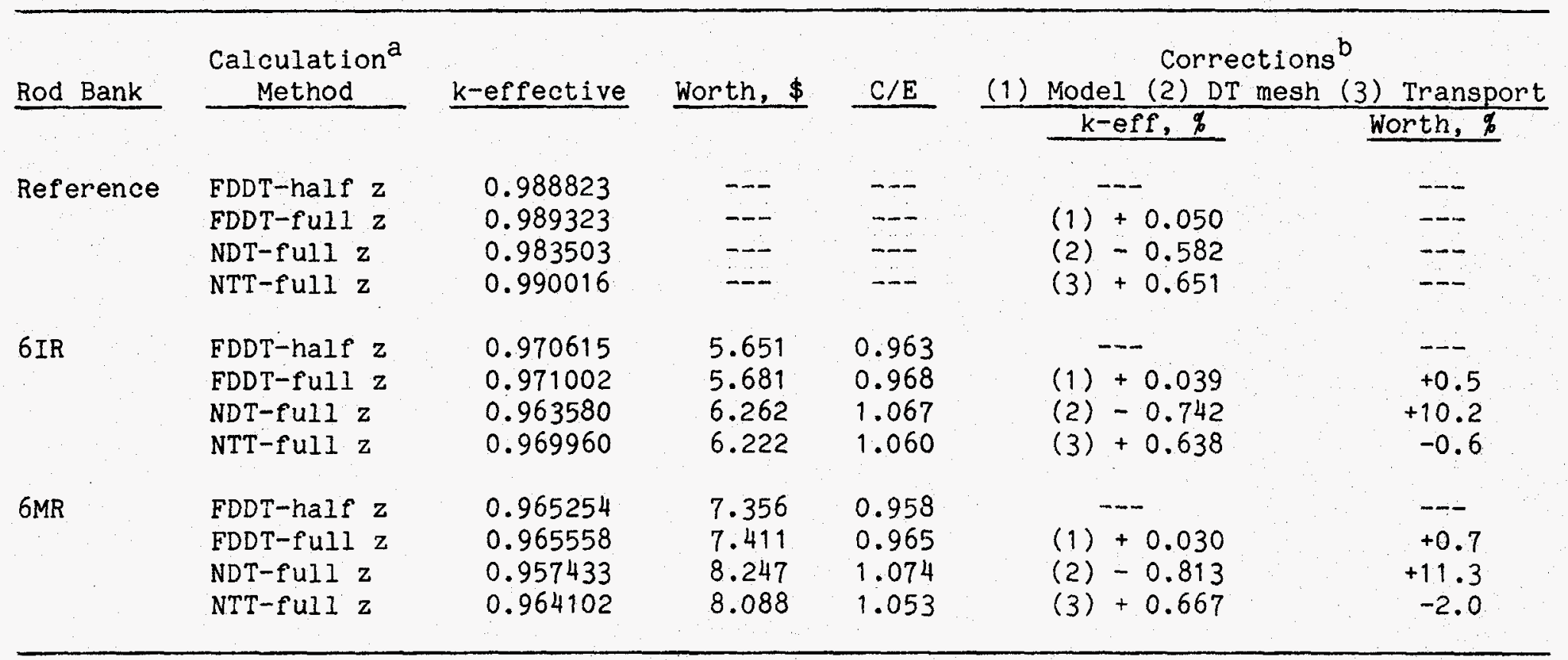

$a_{\text {FDDT }}=$ finite difference diffusion, NDT = nodal diffusion, NTT = nodal transport.

b(1) Model correction half-z to full-z, (2) FDDT mesh $55 \mathrm{~mm}$ to effective fine mesh in NDT,

(3) fine-mesh diffusion (NDT) to transport (NTT). 
TABLE 3.5 Comparison of Control Rod Worths in ZPPR-17B and ZPPR-17C

\begin{tabular}{|c|c|c|c|c|c|}
\hline $\begin{array}{c}\text { Control } \\
\text { Rods } \\
\end{array}$ & Core & $\begin{array}{l}\text { Measured } \\
\text { Worth, } \$\end{array}$ & $\begin{array}{c}\text { Total } \\
\text { Uncertainty, } \% \\
\end{array}$ & $\begin{array}{l}\text { FDDT } \\
\mathrm{C} / \mathrm{E} \\
\end{array}$ & $\begin{array}{l}\text { NTT } \\
\mathrm{C} / \mathrm{E} \\
\end{array}$ \\
\hline $6 \mathrm{IR}$ & $\begin{array}{l}17 \mathrm{~B} \\
17 \mathrm{C}\end{array}$ & $\begin{array}{l}5.822 \\
5.869\end{array}$ & $\begin{array}{l}0.87 \\
0.88\end{array}$ & $\begin{array}{l}0.954 \\
0.968\end{array}$ & $\begin{array}{l}1.054 \\
1.060\end{array}$ \\
\hline $6 \mathrm{MR}$ & $\begin{array}{l}17 \mathrm{~B} \\
17 \mathrm{C}\end{array}$ & $\begin{array}{l}8.318 \\
7.682\end{array}$ & $\begin{array}{l}0.87 \\
0.87\end{array}$ & $\begin{array}{l}0.968 \\
0.965\end{array}$ & $\begin{array}{l}1.045 \\
1.053\end{array}$ \\
\hline $5 \mathrm{MR}$ & $\begin{array}{l}17 \mathrm{~B} \\
17 \mathrm{C}\end{array}$ & $\begin{array}{l}6.537 \\
6.045\end{array}$ & $\begin{array}{l}0.87 \\
0.89\end{array}$ & $\begin{array}{l}0.977 \\
0.969^{a}\end{array}$ & $\cdots$ \\
\hline
\end{tabular}

$a_{\text {Assuming }}+0.7 \%$ modelling correction for 6MR (Table 3.4). 


\section{REVISION TO THE MCCRUNCH CODE (D. A. Tate and P. J. Collins)}

The MCCRUNCH code is used to obtain experimental reactivities by the modified source-multiplication method from analysis of the countrates from a set of sixty-four fission chambers. Calculation input is provided for the detector efficiencies and effective source ratios but because of the large number of detectors, the experimental reactivity is insensitive to the absolute accuracy of the calculated values.

The best estimates of reactivity and uncertainty are obtained from a Iinear least-squares fit (LSFIT) of the reactivity estimates for each detector versus the calculated efficiency ratios. Up until now, the reactivities in the fit were weighted with the statistical uncertainty for each detector and the most deviant results were rejected successively until a value for chi-square per degree of freedom which was less than 1.3 was obtained. This process works extremely well in many cases especially those involving a perturbation in a local region of the core. However, situations arise when the linearity assumption is less valid. These have been noted recently in analysis of the small space-reactor experiments having relatively few detectors in the core region and in the large cores when many control rods are inserted so that few detectors are sufficiently far removed from the perturbation.

In these cases, it frequently happens that results which are less than two standard deviations from the line fit are rejected and that the statistical uncertainty estimate is unduly optimistic.

The revised code takes into account uncertainties in the calculated efficiency ratios. It is assumed that the uncertainty is proportional to the deviation of the efficiency ratio $\left(\varepsilon\right.$ ) from unity. An uncertainty $\sigma_{\varepsilon}$ is defined so that

$$
\sigma_{\varepsilon}=f(1-\varepsilon)
$$

where $f$ is a factor to be determined. The code makes an initial leastsquares fit and rejects any results which are deviant from the line by more than $3.6 \sigma$. This value is chosen so that there is less than a $1 \%$ 
probability of rejecting a valid data point (ZPR-TM-365). The statistical uncertainty from the detector countrates $\left(\sigma_{S}\right)$ is augmented by $\sigma_{\varepsilon}$ as

$$
\sigma^{2}=\sigma_{s}^{2}+\sigma_{\varepsilon}^{2}
$$

Then the value of $f$ is set at 0.2 , a new LSFIT performed and the value of chi-square calculated. The value of $f$ is incremented by 0.1 until the chisquare value is less than 1.47 with no more results being rejected. The final reactivity is obtained from the fit for an efficiency ratio of unity and the uncertainty is calculated from the covariance matrix of the $f$ it.

In a few cases, where statistical uncertainties are relatively low, the initial value of $f$ may yield a chi-square estimates less than unity. In these cases, the value is successively halved (with a lower limit of 0.005 ) until chi-square is greater than unity. Finally, upon attaining an acceptable chi-square, the detectors initially rejected are re-examined with the current value of $f$ and included in a final fit if acceptable.

The chi-square test of 1.47 has been changed from the previous value of 1.3 to correspond to the $99 \%$ probability criterion. For fewer than 60 detectors the code will revise this value from a chi-square table built in to the code.

Although the statistical uncertainties are increased by the revised method, sometimes by a factor of two, it is believed that the values are more realistic. Comparison of the results were made for the ZPPR-17 control rod analysis. The worths usually changed by only a few tenths of a percent. For a localized perturbation, such as a single control rod, the least squares $f$ it was always excellent with only one or two results being aberrant when detector statistics were not augmented. At the other extreme, for cases with many rods inserted, the original LSFIT rejected as many as 30 results and the worths changed by $0.8 \%$.

Two changes have also been made in the uncertainty estimates for the analysis using a 3.6 o rejection criterion from the average (pass $\# 2$ in MCCRUNCH) (i) the standard deviation of the mean reactivity has been 
multiplied by the square root of chi-square to obtain an unbiased estimate of the variance ( $i i$ ) the lower limit of the uncertainty in calculated source ratio has been increased to 0.03 (from 0.01 ) to correspond to the value used in the LSFIT analysis. 
5. REVISIONS TO SODIUM VOID WORTH MEASUREMENTS IN ZPPR-17A (R. W. Goin)

Results for measurements of the worth of sodium removal in ZPPR-17A were reported in ANL-ZPR-476, p. 44. Omitted from Table 6.3 of this report were results from self-contained-oscillator measurements in matrix location 148-39, and the results for 148-43 were mislabeled. The corrected table is given in Table 5.1. Statistical uncertainties of about 0.0015 cents are representative of all measurements reported in Table 6.3 and 6.4 in ANL-ZPR-476. 
TABLE 5.1 Axial Sodium Worth Profiles, in Cents, in Core Locations with Internal Blanket in ZPPR-17A

\begin{tabular}{|c|c|c|c|c|c|c|}
\hline Location & $148-49$ & $148-49$ & $147-43$ & $147-39$ & $148-35$ & $148-34$ \\
\hline $\begin{array}{c}\text { Displacement, } \\
\text { Inches } \\
\end{array}$ & $\begin{array}{l}\text { Voided } \\
\text { Zone } \\
\end{array}$ & $\begin{array}{l}\text { Flooded } \\
\text { Zone } \\
\end{array}$ & & & & \\
\hline 0 & -0.0733 & -0.0599 & -0.0657 & -0.0665 & -0.0714 & -0.0557 \\
\hline 1 & -0.0625 & -0.0473 & -0.0527 & -0.0561 & -0.0536 & -0.0472 \\
\hline 2 & -0.0544 & -0.0402 & -0.0454 & -0.0427 & -0.0476 & -0.0365 \\
\hline 3 & -0.0445 & -0.0307 & -0.0371 & -0.0398 & -0.0376 & -0.0273 \\
\hline 4 & -0.0373 & -0.0211 & -0.0287 & -0.0288 & -0.0267 & -0.0142 \\
\hline 5 & -0.0323 & -0.0215 & -0.0208 & -0.0195 & -0.0229 & -0.0082 \\
\hline 6 & -0.0278 & -0.0146 & -0.0212 & -0.0117 & -0.0097 & -0.0031 \\
\hline 7 & -0.0226 & -0.0118 & -0.0137 & -0.0072 & -0.0024 & 0.0079 \\
\hline 8 & -0.0167 & -0.0054 & -0.0054 & -0.0003 & 0.0054 & 0.0205 \\
\hline 9 & -0.0040 & -0.0042 & -0.0040 & 0.0077 & 0.0148 & 0.0284 \\
\hline 10 & -0.0036 & 0.0008 & 0.0035 & 0.0110 & 0.0250 & 0.0316 \\
\hline 11 & 0.0076 & 0.0067 & 0.0086 & 0.0190 & 0.0342 & 0.0407 \\
\hline 12 & 0.0162 & 0.0165 & 0.0127 & 0.0274 & 0.0403 & 0.0529 \\
\hline 13 & 0.0177 & 0.0188 & 0.0176 & 0.0258 & 0.0435 & 0.0541 \\
\hline 14 & 0.0236 & 0.0275 & 0.0197 & 0.0395 & 0.0464 & 0.0508 \\
\hline 15 & 0.0302 & 0.0336 & 0.0296 & 0.0369 & 0.0494 & 0.0577 \\
\hline 16 & 0.0306 & 0.0284 & 0.0321 & 0.0428 & 0.0545 & 0.0551 \\
\hline 17 & 0.0304 & 0.0266 & 0.0276 & 0.0406 & 0.0485 & 0.0524 \\
\hline 18 & 0.0321 & 0.0310 & 0.0284 & 0.0443 & 0.0432 & 0.0502 \\
\hline 19 & 0.0257 & 0.0266 & 0.0274 & 0.0348 & 0.0398 & 0.0378 \\
\hline 20 & 0.0216 & 0.0188 & 0.0204 & 0.0252 & 0.0305 & 0.0357 \\
\hline 21 & 0.0189 & 0.0146 & 0.0170 & 0.0183 & 0.0210 & 0.0221 \\
\hline 22 & 0.0073 & 0.0116 & 0.0058 & 0.0172 & 0.0173 & 0.0198 \\
\hline 23 & 0.0086 & 0.0067 & 0.0018 & 0.0098 & 0.0136 & 0.0175 \\
\hline 24 & 0.0059 & 0.0027 & 0.0033 & 0.0065 & 0.0081 & 0.0068 \\
\hline 25 & -0.0002 & 0.0024 & 0.0028 & 0.0068 & 0.0009 & 0.0011 \\
\hline 26 & 0.0000 & 0.0000 & 0.0000 & 0.0000 & 0.0000 & 0.0000 \\
\hline
\end{tabular}




\section{MEASUREMENTS AND ANALYSIS OF SODIUM VOID REACTIVITY IN ZPPR-15C \\ (S. B. Brumbach and P. J. Collins)}

ZPPR-15C was a benchmark metal-fuelled core with approximately equal fissile loadings of plutonium and uranium. As in ZPPR-15B and 15D, the inner core contained zirconium at about $10 \%$ by weight of the fuel and the central 148 drawers in each half of the assembly comprised of "symmetriczirconium" zone utilizing the thinner zirconium and depleted uranium stock. Sodium void measurements in the central zone provide an interesting comparison with results from the all-plutonium fuelled core 15B and from the all-uranium fuelled zone in $2 P P R-15 D$.

The reactivities due to sodium voiding were measured in two axial steps in the central 52 drawers of each assembly half. The measurements were made by replacing sodium-filled cans by empty cans. Reactivities were determined by subcritical source multiplication using the countrates in the reference configuration. The reactivity of the reference was established by inverse kinetics analysis of the power history following a rod drop.

The subcritical reference for $Z P P R-15 C$ is described in $Z P R-T M-471$, p. 5. This reference was established on April 22, 1986, as loading 165 prior to the sodium void experiments. Since the voiding reactivity was positive, the reactivity of the reference was first decreased by converting double-fuel-column drawers (master 934 and 928) to single-fuel-column drawers (master 921 and 922) in matrix locations 157-34 and 145-48 and the symmetric locations, constituting loading 167 . This fuel reduction step is called the "despike". The sodium voiding was done in the central 52 drawers in two axial steps, $\pm 203 \mathrm{~m}$ and \pm 457 about the midplane, in reactor loadings 168 and 169. The sodium was added back in a "reflood" step in loading 170 , to check the reference reactivity after the drawer movements. All drawers in the void zone contained one column of fuel; 28 Pu-fueled drawers and 24 U-fueled drawers per half. Two drawers with fission chambers (master 716), which contained three columns of sodium instead of four columns in the regular drawers, were voided along with the other drawers (voided master 718). The experiments were made between April 24, 1986 and April 28, 1986, in reactor runs 293 to 295. The fission chamber data were 
recorded on the 64-detector files 155 to 158. The reactivity calibration was made in the subcritical reference loading in run 290 (64-detector file 153, inverse kinetics data file 13). The reactivity of this reference was $-11.268 \pm 0.089 \phi$. The reactivities of the void reference (despike) and of the void steps were determined relative to this reference by analysis of the fission chamber countrates with the MCCRUNCH code. One detector (number 41 ) and one/two thermocouples (number 1 and 76 ) were inoperative during the measurements and were excluded from the data processing.

Because of the small reactivity changes, the data analys is in MCCRUNCH assumed detector efficiencies and effective source ratios of unity. Detectors predicting reactivities which were more than 3.6 standard deviations from the mean were rejected. At least 45 of the original 63 detectors were retained by this process. The reactivity analysis from the MCCRUNCH code is summarized in Table 6.1. Upon "reflood" after the void steps, the reactivity of the despike reference differed by $0.15 \phi$. This difference is attributed to movement of plates in the drawers during the voiding/reflood operations. Since the precise origin of the reactivity increment is unknown, we attribute an additional 10 uncertainty component of $0.15 \notin$ to each void step reactivity.

The temperature and interface-gap reactivity coefficients were not measured in $2 P P R-15 C$. For the data processing, the coefficients were approximated in the following way. The temperature coefficient was taken as the mean from $Z P P R-15 B$ and $Z P P R-15 D$ results in $\triangle k$ units and divided by $B$-effective for $Z P P R-15 C$ to convert to dollar units. The gap coefficient used the value measured in $Z P P R-15 B$ scaled by the ratio of $B$-effectives. The ${ }^{241} \mathrm{Pu}$ decay coefficient was not calculated for ZPPR-15C. A value was obtained from the $Z P P R-15 \mathrm{~A}$ results scaled by the ratio of ${ }^{241} \mathrm{Pu}$ mass to the total fissile mass and by the $\beta$-effective ratio. The following coefficients were used in the MCCRUNCH code:

Temperature coefficient

Gap coefficient

${ }^{241} \mathrm{Pu}$ decay coefficient

$$
\begin{aligned}
& (-0.00575 \pm 0.0011) \$ \mathrm{~K}^{-1} \\
& (-0.000852 \pm 0.00013) \$ \mathrm{mil}^{-1} \\
& (-0.000063 \pm 0.000003) \$ \mathrm{day}^{-1}
\end{aligned}
$$


These estimates are sufficiently accurate in view of the small reactivity adjustments required.

The reactivities of the void steps are given in Table 6.2. The mass of steel changed a little with each void step due to differences in masses of sodium cans and empty cans. Corrections for the steel changes were taken from calculations for ZPPR-15A (scaled by the $\beta$-effective ratio) and an uncertainty of $30 \%$ of the correction was included in the statistical uncertainty for each step.

Calculations of sodium void reactivity were made using the xyz model of ZPPR-15C with nodal diffusion solutions and 21 group cross sections. The cross sections were taken from the ZPPR-15B library for plutonium-fuelled drawers and from the ZPPR-15D library for uranium-fuelled drawers (ANL-ZPR-473, p. 4). These data included cross sections processed for the heterogeneity in the voided cells and anisotropic diffusion coefficients for the voided cells. The fission chamber drawers were treated as normal drawers in the calculation model. The masses of sodium voided were slightly different from those in the experiment and were allowed for by expressing results in units of $\phi / \mathrm{kg}(\mathrm{Na})$. A value for $\beta$-effective of $0.5207 \%$ was used to convert calculations to cent units (ANL-ZPR-473, p. 28).

The calculated void reactivities are compared with measured values in Table 6.3. For the central zone $(0 \pm 203 \mathrm{~mm})$, the calculation overestimates the void reactivity by about $17 \%$. The value of $C-E$ $(+0.134 \phi / \mathrm{kg})$ falls between that for the all plutonium-fuelled core $Z P P R-15 B$ $(+0.145 \phi / \mathrm{kg})$ and that for the $90 \%$ uranium-fuelled ZPPR-15D $(0.116 \phi / \mathrm{kg})$. 
TABLE 6.1. Data Processing for ZPPR-15C Sodium Void Experiments

\begin{tabular}{|c|c|c|c|c|c|c|}
\hline Measurement & $\begin{array}{l}\text { Data } \\
\text { File }\end{array}$ & $\begin{array}{c}\text { No. of } \\
\text { Detectors }\end{array}$ & $x^{2 c}$ & Reactivity, $\phi^{\mathrm{a}}$ & $\sigma_{s}, \phi^{d}$ & $\sigma_{c}, \phi^{e}$ \\
\hline Despike & 155 & 57 & 3.21 & -24.57 & 0.070 & 0.222 \\
\hline $203 \mathrm{~mm}$ Void & 156 & 49 & 4.95 & -8.58 & 0.058 & 0.090 \\
\hline $457 \mathrm{~mm}$ Void & 157 & 45 & 3.82 & -10.17 & 0.053 & 0.103 \\
\hline Reflood & 158 & 58 & 3.81 & -24.72 & 0.079 & 0.223 \\
\hline
\end{tabular}

a Reactivities are derived relative to the subcritical reference for which detector counts are recorded on File 153. The reactivity of this configuration was determined by inverse kinetics (File 13) to be $-11.268 \pm 0.089 \phi$.

${ }^{b}$ Number of detectors remaining after rejection of those giving reactivities which were more than 3.6 standard deviations from the mean.

${ }^{\mathrm{C}}$ Value of chi-square for remaining detectors using counter statistics.

$\mathrm{d}_{\text {Statistical uncertainty. }}$

${ }^{e}$ Correlated uncertainty. 
TABLE 6.2. Experimental Reactivity Changes for Sodium Void Steps in ZPPR-15C

\begin{tabular}{|c|c|c|c|c|c|c|c|c|}
\hline Step $^{a}$ & $\begin{array}{r}\text { Mass Sodium } \\
\text { Voided, kg } \\
\end{array}$ & $\begin{array}{r}\text { Mass Steel } \\
\text { Added, kg } \\
\end{array}$ & $\begin{array}{c}\text { Measured } \\
\text { Reactivity } \\
\text { Change, } \notin \\
\end{array}$ & $\begin{array}{c}\text { Steel } \\
\text { Correction, } \phi \\
\end{array}$ & $\begin{array}{c}\text { Corrected } \\
\text { Reactivity, } \varnothing \\
\end{array}$ & $\sigma_{s}, \phi^{d}$ & $\sigma_{c}, \phi^{\mathrm{d}}$ & $\begin{array}{l}\text { Specific } \\
\text { Reactivity } \\
\phi / \mathrm{kg} \text { (Na) }\end{array}$ \\
\hline $\begin{array}{l}0-203 \mathrm{~mm} \\
0-457 \mathrm{~mm} \\
203-457 \mathrm{~mm}\end{array}$ & $\begin{array}{l}20.804 \\
46.080 \\
25.276\end{array}$ & $\begin{array}{l}0.366 \\
2.395 \\
2.029\end{array}$ & $\begin{array}{l}15.99 \\
14.40 \\
-1.59\end{array}$ & $\begin{array}{l}+0.07 \\
+0.32 \\
+0.25\end{array}$ & $\begin{array}{r}16.06 \\
14.72 \\
-1.34\end{array}$ & $\begin{array}{l}0.091 \\
0.088 \\
0.079\end{array}$ & $\begin{array}{l}0.132 \\
0.119 \\
0.013\end{array}$ & $\begin{array}{r}0.772 \\
0.319 \\
-0.051\end{array}$ \\
\hline
\end{tabular}

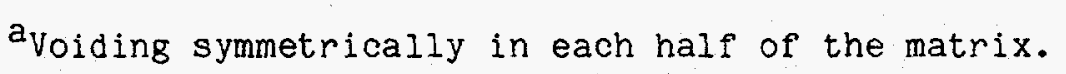

bstatistical uncertainty including $30 \%$ of the steel correction, but not including reproducibility uncertainty $(0.13 \phi)$.

${ }^{c}$ Correlated uncertainty includes reference reactivity and source ratio uncertainties. 
TABLE 6.3. Calculated Reactivities for Sodium Voiding in ZPPR-15C and Comparison with Experiment

\begin{tabular}{|c|c|c|c|c|c|c|}
\hline Step ${ }^{a}$ & $\begin{array}{c}\text { Calculated } \\
k_{\text {eff }}\end{array}$ & $\begin{array}{l}\text { Calculated } \\
\text { Worth, } \phi^{c}\end{array}$ & $\begin{array}{c}\text { Mass } \\
\text { Sodium } \\
\text { Voided, kg }\end{array}$ & $\begin{array}{l}\text { Specific } \\
\text { Reactivity } \\
\phi / \mathrm{kg}(\mathrm{Na})\end{array}$ & $\begin{array}{c}C-E \\
\phi / \mathrm{kg}(\mathrm{Na})\end{array}$ & $\begin{array}{l}\text { Experimental } \\
\text { Uncertainty } \\
\quad \not / \mathrm{kg}(\mathrm{Na})\end{array}$ \\
\hline $\begin{array}{r}0-203 \mathrm{~mm} \\
0-457 \mathrm{~mm} \\
203-457 \mathrm{~mm}\end{array}$ & $\begin{array}{c}0.992070 \\
0.992053 \\
-\end{array}$ & $\begin{array}{r}18.63 \\
18.30 \\
-1.33\end{array}$ & $\begin{array}{l}20.566 \\
47.123 \\
26.557\end{array}$ & $\begin{array}{l}+0.9060 \\
+0.3884 \\
-0.0501\end{array}$ & $\begin{array}{l}+0.134 \\
+0.069 \\
-0.001\end{array}$ & $\begin{array}{l}0.011 \\
0.005 \\
0.007\end{array}$ \\
\hline
\end{tabular}

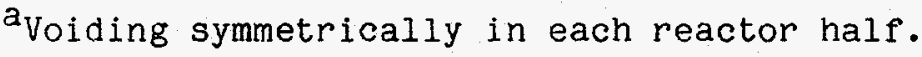

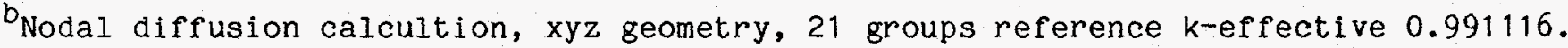

$c_{\text {Using }} \beta$-effective $=0.005207$

$d_{\text {Experimental uncertainty includes reproducibility }(0.15 \phi)}$ 
7. MEASUREMENT AND ANALYSIS OF SODIUM VOID REACTIVITY IN THE HIGH-ZR ZONE OF ZPPR-15 (S. B. Brumbach and P. J. Collins)

Assemblies $Z P P R-15 A$ and $Z P P R-15 B$ were plutonium-metal-fueled benchmark LMR cores differing principally in the addition of zirconium to the inner core of $\mathrm{ZPPR}-15 \mathrm{~B}$. Several intermediate steps were made in the conversion of $15 \mathrm{~A}$ to 15B to measure reactivity effects of exchanging zirconium for steel and zirconium for uranium. The first step exchanged zirconium for uranium and the second step exchanged zirconium for stainless steel in a central zone of 132 drawers in each half. At the end of the second step the net change was replacement of two $1 / 16$ inch columns of depleted uranium plus two $1 / 16$ inch columns of stainless steel by two $1 / 8$ inch columns of zirconium. This configuration was called the "High-Zirconium Zone" of ZPPR-15. The zirconium content was about $20 \%$ of the heavy metal by weight, and was twice that of the central zone of the $Z P P R-15 B$ reference. Drawer loadings for the central zone of ZPPR-15A, of the High-Zr Zone and of ZPPR-15B are shown in Figures 7.1 , 7.2, and 7.3. Atomic densities are compared in Table 7.1. Measurements made in the High-Zr Zone were central sodium void ( $\pm 203 \mathrm{~mm}$ from the midplane) and reaction rate ratios.

Several changes in the ZPPR-15A fuel loading were necessary in constructing the reference for sodium voiding in the High-Zr zone. These changes involved the exchange of single-fuel column and double-fuel column drawers. Loading 76 was the subcritical reference for sodium voiding. The sodium void measurement was made in the central 52 drawers of the High-Zr zone (loading 77 reactor run 152). The reactivity of this loading was measured by inverse kinetics analysis of a rod drop to be $-32.36 \phi \pm 0.26 \%$, with countrates for the 64-fission chambers recorded on file 76 . The reactivities of the zone with sodium in were determined relative to this reference reactivity by the subcritical multiplication method using the McCRUNCH code. The sodium-in configurations were built immediately before the reference (loading 76 , run 151, data file 75) and immediately afterwards (loading 78, run 153, data file 77).

The data processing with MCCRUNCH was made with unit detector efficiencies and unit source ratio. Temperature, interface gap, and ${ }^{241} \mathrm{Pu}-$ 
decay reactivity coefficients were the same as used for ZPPR-15A. Three detectors yielding reactivity estimates which were more than 3.6 standard deviations from the mean (relative to detector statistical uncertainties) were rejected in the analysis. The data processing is summarized in Table 7.2

The difference in reactivities before and after the voiding was $0.47 \$$ or $1.28 \%$. This is attributed to movements of materials during the operations of opening of the halves of the assembly, removal and replacement of drawers and exchange of sodium-filled cans with void cans and vice versa. The mean reactivity is used and an additional uncertainty of $1.28 \%(1 \sigma)$ is attributed to the measurement.

A small correction, $+0.11 \phi$, is made to the measured void reactivity due to the differences in steel mass between the cans of the sodium-filled plates and the void cans. The correction was obtained from calculations made for the void replacements in $Z P P R-15 A$ ( $Z P R-7 M-469, p .78)$. An uncertainty of $30 \%$ of the calculated correction, $0.033 \phi$, is assumed. The mass of sodium voided in the experiment was $20.804 \mathrm{~kg}$.

A calculation was made with the nodal diffusion method with an xyz model and ENDF/B-V.2 data collapsed to 21 energy groups. The microscopic cross sections were generated for the ZPPR-15B core and not reprocessed for the heterogeneity of the High-Zr cell. A nodal perturbation edit was made to obtain non-leakage and leakage components. The mass of sodium voided in the calcuations, $20.566 \mathrm{~kg}$, was slightly different to that in the measurement because atom densities were calculated for the full core height and not specifically for the $203 \mathrm{~mm}$ void region.

Measured and calculated void reactivities are compared in Table 7.3. The total worth from nodal perturbation theory is only $0.4 \%$ less than that by $k$ difference. Calculation overestimated the void worth by $16 \%$. 
TABLE 7.1 Comparison of Atom Densities for Sodium Void Zones in ZPPR-15A, 15B, and High-Zr Zone

\begin{tabular}{|c|c|c|c|c|}
\hline \multirow[b]{2}{*}{ Isotope ${ }^{a}$} & \multicolumn{2}{|c|}{$2 P P R-15 \mathrm{~A}^{\mathrm{b}}$} & \multirow{2}{*}{$\begin{array}{l}\text { ZPPR-15B } \\
\text { Master } 138 \\
\end{array}$} & \multirow{2}{*}{$\begin{array}{l}\text { High } \mathrm{Zr}^{\mathrm{c}} \text { Zone } \\
\text { Master } 128 \\
\end{array}$} \\
\hline & Master 101 & Master 102 & & \\
\hline C & 0.0000914 & 0.0001000 & 0.0000785 & 0.0000785 \\
\hline 0 & 0.0000006 & 0.0000006 & 0.0000006 & 0.0000006 \\
\hline $\mathrm{Na}$ & 0.0083220 & 0.0083220 & 0.0083220 & 0.0083220 \\
\hline $\mathrm{Si}$ & 0.0003738 & 0.0005290 & - & 0.0003639 \\
\hline AI & 0.0000061 & 0.0000061 & 0.0000061 & 0.0000061 \\
\hline $\mathrm{Mn}$ & 0.0005934 & 0.0006141 & 0.0005139 & 0.0005139 \\
\hline $\mathrm{Cr}$ & 0.0064913 & 0.0066395 & 0.0056630 & 0.0056630 \\
\hline $\mathrm{Fe}$ & 0.0231873 & 0.0228917 & 0.0200066 & 0.0200066 \\
\hline $\mathrm{Ni}$ & 0.0029190 & 0.0029134 & 0.0025274 & 0.0025274 \\
\hline $\mathrm{Cu}$ & 0.0000315 & 0.0000315 & 0.0000315 & 0.0000315 \\
\hline $\mathrm{Zr}$ & - & - & 0.0020955 & 0.0044234 \\
\hline Mo & 0.0002388 & 0.0002388 & 0.0002388 & 0.0002388 \\
\hline U235 & 0.0000164 & 0.0000164 & 0.0000164 & 0.0000108 \\
\hline U238 & 0.0073554 & 0.0073549 & 0.0073554 & 0.0049558 \\
\hline Pu238 & 0.0000005 & 0.0000005 & 0.0000005 & 0.0000005 \\
\hline Pu239. & 0.0008844 & 0.0008844 & 0.0008844 & 0.0008844 \\
\hline Pu240 & 0.0001169 & 0.0001169 & 0.0001169 & 0.0001169 \\
\hline Pu241. & 0.0000078 & 0.0000078 & 0.0000076 & 0.0000076 \\
\hline PU242 & 0.0000019 & 0.0000019 & 0.0000019 & 0.0000019 \\
\hline Am241 & 0.0000100 & 0.0000100 & 0.0000103 & 0.0000103 \\
\hline $\mathrm{P}$ & 0.0000186 & 0.0000192 & 0.0000159 & 0.0000156 \\
\hline$S$ & 0.0000037 & 0.0000107 & 0.0000082 & 0.0000047 \\
\hline $\mathrm{Cl}$ & 0.0000003 & 0.0000003 & 0.0000003 & 0.0000003 \\
\hline $\mathrm{Ca}$ & 0.0000019 & 0.0000019 & 0.0000019 & 0.0000019 \\
\hline Co & 0.0000014 & 0.0000014 & 0.0000014 & 0.0000014 \\
\hline
\end{tabular}

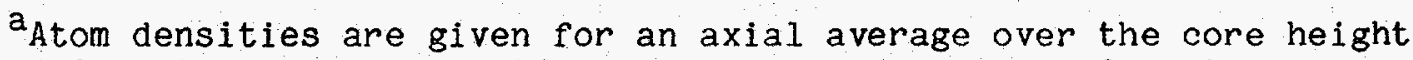
$( \pm 8$ in.). The actual densities in the void zone ( \pm 8 in.) were slightly different due to the variation in piece lengths.

${ }^{b}$ Sodium voiding in $Z P P R-15 A$ was done in a central zone of 148 matrix positions. The inner 52 positions were master 101 and the other 96 positions were master 102 .

${ }^{\circ}$ Sodium voiding in $\mathrm{ZPPR}-15 \mathrm{~B}$ and the High $\mathrm{Zr}$ Zone was done in a central zone of 52 matrix positions. 
TABLE 7.2 Data Processing for the Sodium Void Measurement in the High-Zr Zone of ZPPR-15

\begin{tabular}{lcc}
\hline & & \\
Reactor loading & 76 & 78 \\
Reactor run & 151 & 153 \\
Data File & 75 & 77 \\
Temperature, ${ }^{\circ} \mathrm{C}$ & 24.67 & 24.45 \\
Temperature correction, $\phi$ & -0.09 & -0.32 \\
Interface gap, mil & 73.9 & 73.6 \\
Gap correction, $\phi$ & -0.04 & -0.08 \\
Date & $11 / 5 / 85$ & $11 / 7 / 85$ \\
Decay correction, $\phi$ & -0.03 & +0.03 \\
Number of detectors & 61 & 61 \\
Reduced chi-square & 1.54 & 1.59 \\
Reactivity change, $\phi$ & -36.12 & -36.59 \\
Statistical 10, $\%$ & 0.134 & 0.166 \\
Correlated $1 \sigma / \%$. & 0.824 & 0.822 \\
Total $10, \%$ & 0.834 & 0.839 \\
\hline
\end{tabular}

The reference reactivity was measured in the voided zone as $-32.36 \notin 0.26 \notin$ in loading 77 , run 152 , data $\mathrm{file} 76$ on $11 / 6 / 85$ with temperature $24.76^{\circ} \mathrm{C}$, interface gap $74.22 \mathrm{mil}$. 
TABLE 7.3 Measured and Calculated Sodium Void Reactivities in the High $\mathrm{Zr}$-Zone of $\mathrm{ZPPR}-15$

Measured Worth

Total Uncertainty

Specific Worth (E)

Calculation:

Reff - Reference

Voided

Calculated worth ${ }^{a}$

Specific worth ${ }^{\mathrm{c}}$

Non-leakage

Leakage

$\mathrm{C} / \mathrm{E}$

$C-E$
$36.35 \not$

$1.53 \%$

$1.747 \varnothing / \mathrm{kg}$ (Na)

0.989641

0.991011

$41.85 \notin$

$2.033 \phi / \mathrm{kg}$ (Na)

$2.241 \notin / \mathrm{kg}$ ( Na)

$-0.215 \notin / \mathrm{kg}(\mathrm{Na})$

1.164

$0.286 \notin / \mathrm{kg}$ (Na)

$a_{\text {Using calculated }} B_{\text {eff }}+0.3361 \%$ 


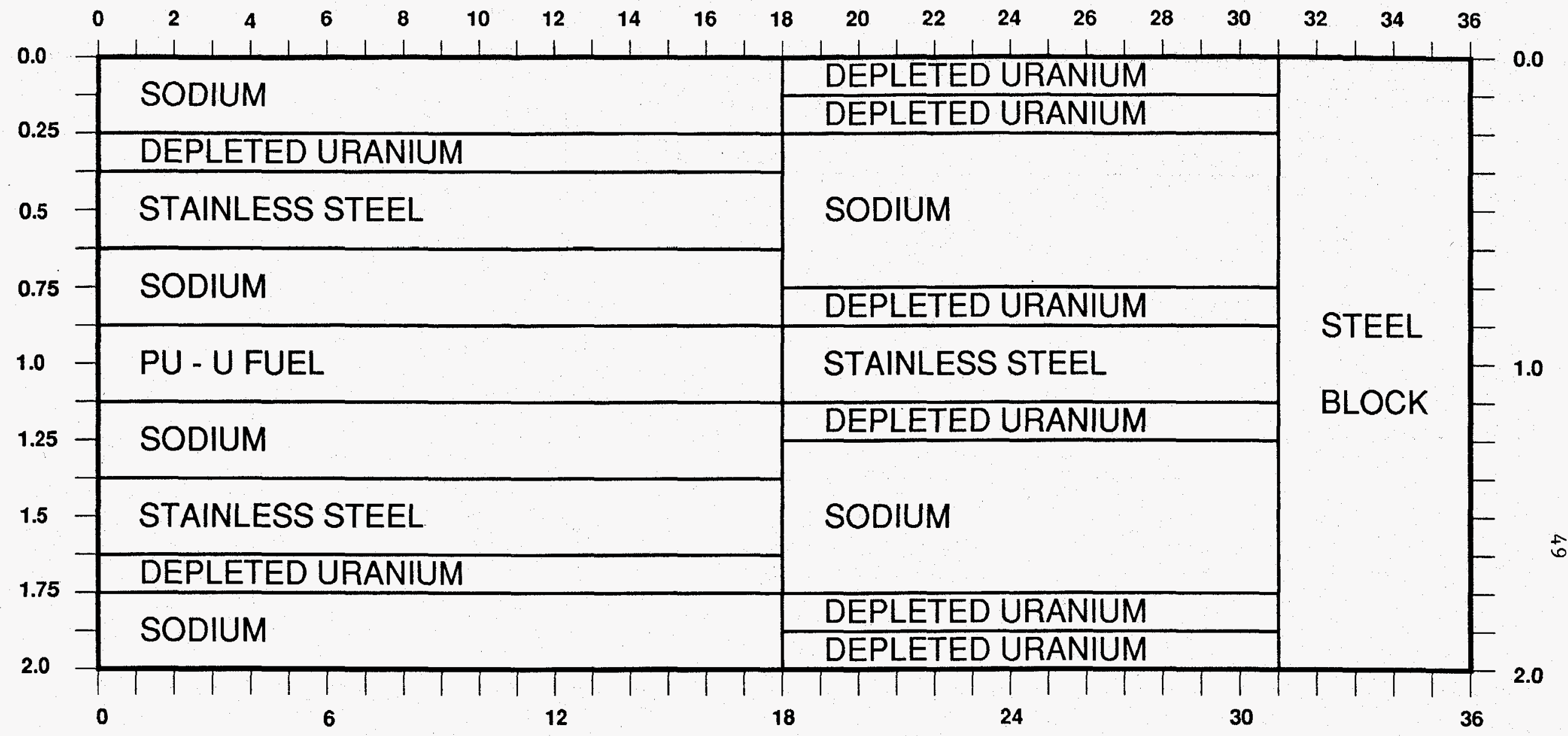

Fig. 7.1 Loading Pattern for Inner Core Drawers in ZPPR-15A 


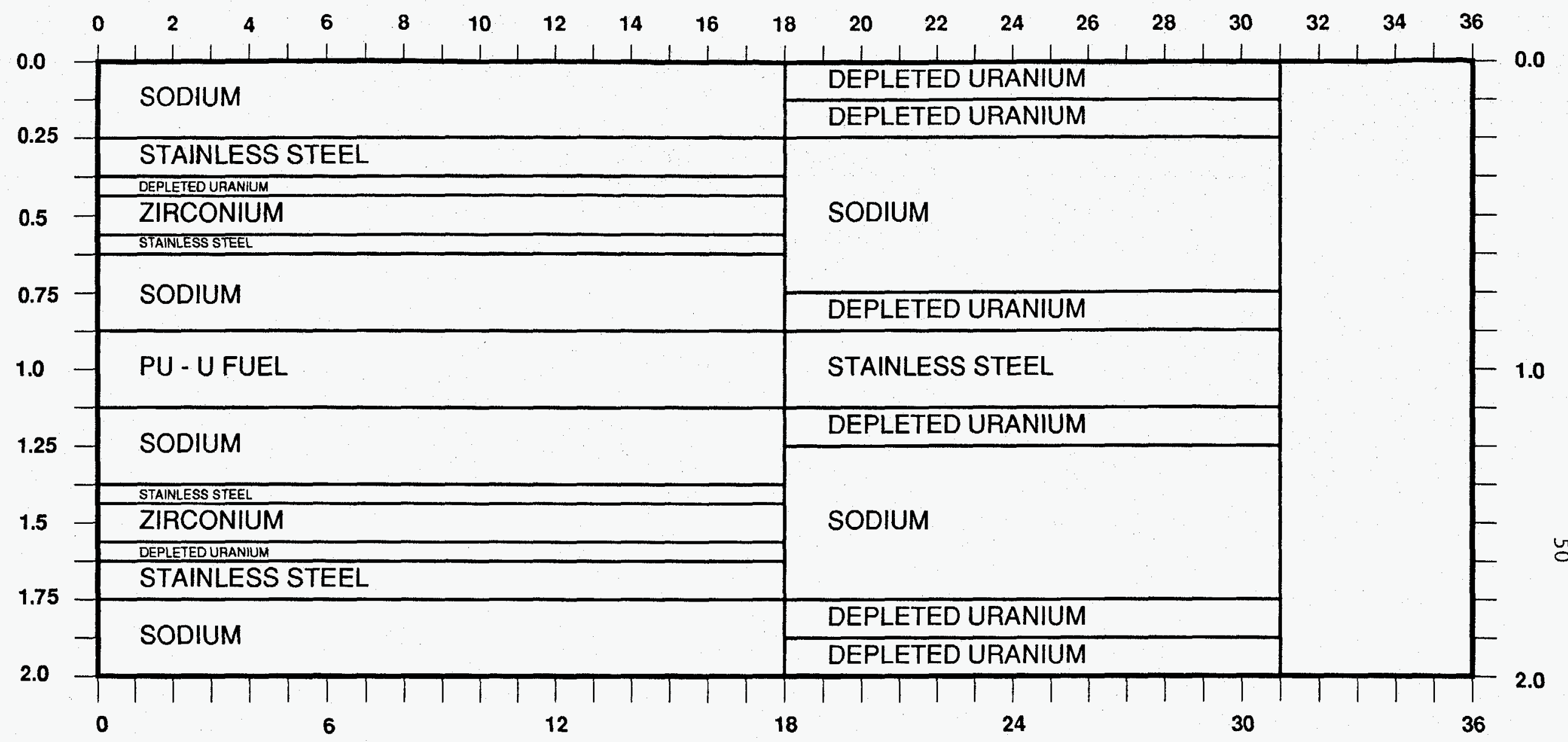

Fig. 7.2 Loading Pattern for Drawers in the High-Zirconium Zone in ZPPR-15 


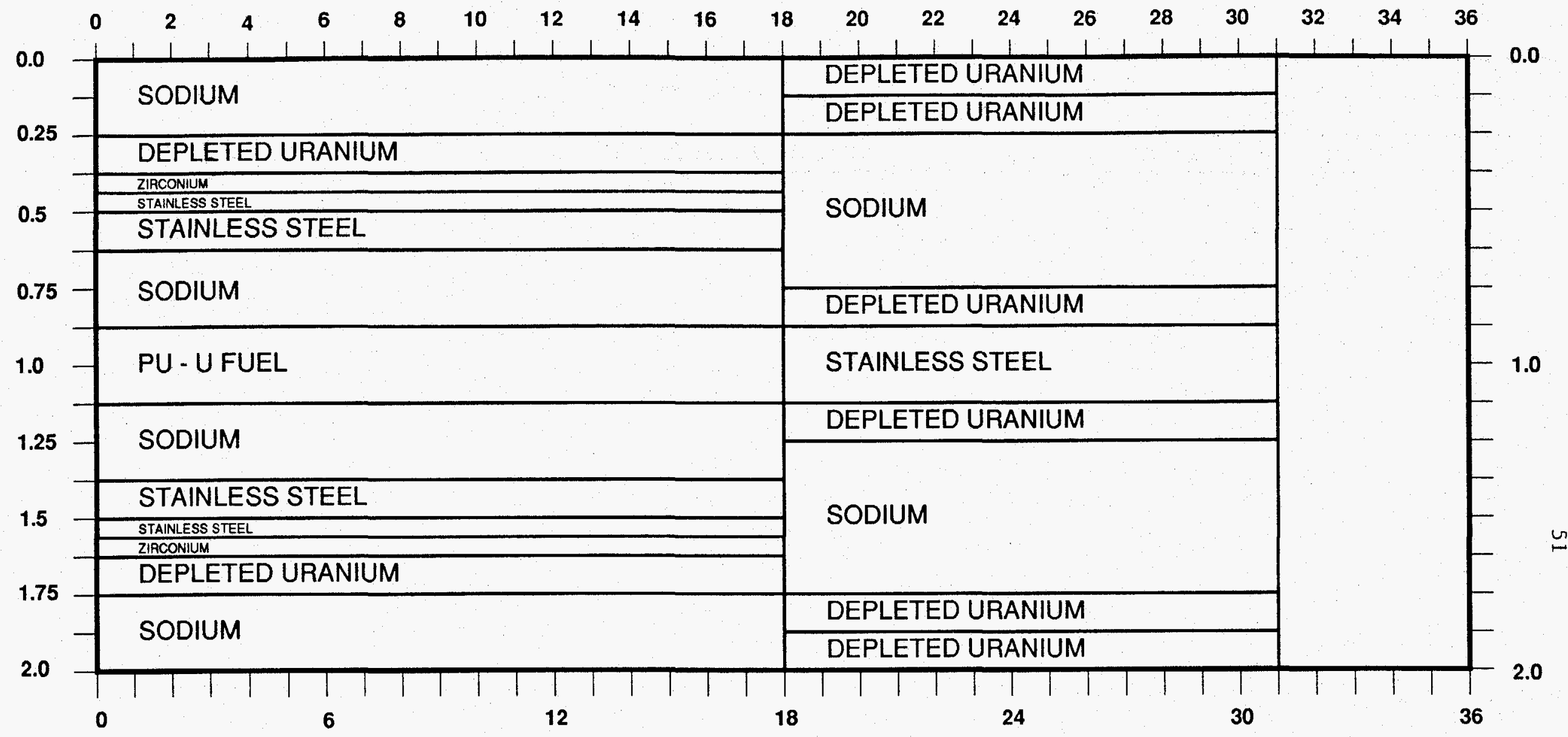

Fig. 7.3 Loading Pattern for Drawers in the Central, Symmetric Zone in ZPPR-15B 
8. SUMMARY OF SODIUM VOID RESULTS IN ZPPR-15 (P. J. Collins)

The sodium void reactivity for a central zone was measured in five different compositions in the ZPPR-15 program. These were (1) the plutonium fueled ZPPR-15A, (2) the plutonium-fueled ZPPR-15B with $10 \% \mathrm{Zr}$, (3) the High$\mathrm{Zr}$ Zone, (4) the 50\%-Pu/50\%-U fueled $Z P P R-15 \mathrm{C}$, and (5) the uranium-fueled central zone of $Z P P R-15 D$.

Calculations for all cases were made with nodal diffusion theory in $x y z$ geometry and ENDF/B-V.2 data. The model for ZPPR-15A used a 28 group section set and the remainder used a 21 group set. Cell heterogeneity calculations, using asymptotic-cell models were made for 15A, 15B, and 15D. Calculations for $15 \mathrm{C}$ used the $15 \mathrm{~B}$ and $15 \mathrm{D}$ data and that for the High-Zr Zone used 15B data.

A comparison of transport $\mathrm{S} 4$ and diffusion results in $\mathrm{rz}$ geometry was made for ZPPR-15B. For the central zone, the transport calculation gave a void reactivity $0.1 \%$ greater than diffusion theory. It is assumed that the difference would also be small in the other cases.

Void reactivites for the five zones are compared in Table 8.1 . Note that between $15 \mathrm{~A}$ and $15 \mathrm{~B}$, zirconium replaces steel. The relative effects of steel and zirconium upon the void reactivity are compared by first order perturbation calculations in ZPR-TM-471, P42. Similarly, between 15B and the High-Zr Zone, zirconium replaces depleted uranium. The last column in Table 8.1 compares the measured void reactivities times calculated $B$-effectives. In this $\Delta \mathrm{k}$ comparison, the void reactivity in $15 \mathrm{C}$ is approximately the average of results for $15 \mathrm{~B}$ and $15 \mathrm{D}$.

While the C/E result for 15D is significantly higher than for the other cases because of the small void reactivity, the C-E result is the lowest of the set. Beyond noting that all the central void reactivities are overestimated by calculation, it is difficult to draw any firm conclusions from the relative results at this stage. The calculated void reactivites are sensitive to cell heterogeneity effects. For example, a calculation for $15 \mathrm{C}$ 
using cross sections for the non-voided uranium cells by mistake gave a negative value for $C-E$. Detailed studies of cell processing would appear to be necessary to give confidence in the results. 
TABLE 8.1 Comparison of Central Sodium Void Reactivities in ZPPR-15

\begin{tabular}{|c|c|c|c|c|c|c|c|c|}
\hline Core (Zone) & Fuel & $\begin{array}{c}\text { Measured } \\
\text { Reactivity } \\
\phi / \mathrm{kg}(\mathrm{Na}) \\
\end{array}$ & $\begin{array}{r}\text { Calculated } \\
\text { Uncertainty } \\
10, \phi / \mathrm{kg} \\
\end{array}$ & $\begin{array}{l}\text { Reactivity } \\
\notin / \mathrm{kg}(\mathrm{Na}) \\
\end{array}$ & $C / E$ & $C-E$ & $\begin{array}{c}\text { Calculated } \\
\text { B-eff }\end{array}$ & $\begin{array}{l}\text { Measured } \\
\text { Reactivity } \times \text {-eff } \\
\end{array}$ \\
\hline $15 \mathrm{~A}$ & $\mathrm{Pu} / \mathrm{U}$ & 1.948 & 0.017 & 2.170 & 1.114 & 0.222 & 0.3357 & 0.6539 \\
\hline $15 \mathrm{~B}$ & $\mathrm{Pu} / \mathrm{U} / \mathrm{Zr}$ & 2.083 & 0.019 & 2.228 & 1.070 & 0.145 & 0.3361 & 0.7001 \\
\hline $\mathrm{High}-\mathrm{Zr}$ & $\mathrm{Pu} / \mathrm{U} / \mathrm{Zr}$ & 1.742 & 0.027 & 2.033 & 1.167 & 0.291 & 0.3361 & 0.5855 \\
\hline $15 \mathrm{C}$ & $\begin{array}{l}50 \% \mathrm{Pu} / \mathrm{U} / \mathrm{Zr} \\
50 \% \mathrm{U} / \mathrm{Zr}\end{array}$ & 0.772 & 0.011 & 0.906 & 1.174 & 0.134 & 0.5207 & 0.4020 \\
\hline $15 \mathrm{D}$ & $\mathrm{U} / \mathrm{Zr}$ & 0.233 & 0.008 & 0.349 & 1.50 & 0.116 & 0.6564 & 0.1529 \\
\hline
\end{tabular}


9. NEUTRON SPECTRUM MEASUREMENTS IN ZPPR-15 (R. W. Goin and J. M. Larson)

Neutron spectra were measured at the core center of ZPPR-15A, B and D and outside the shield zones of $Z P P R-15 B$. The proton recoil technique with both hydrogen and methane-filled counters was used. The basic methodology of the technique was described by Bennett and Yule (E. F. Bennett and T. J. Yule, "Techniques and Analyses of Fast Reactor Neutron Spectroscopy with Proton Recoil Proportional Counters," ANL-7763, 1971). A new data acquisition system and a modified data reduction procedure were used. The energy range covered was approximately $1 \mathrm{keV}$ to $2 \mathrm{MeV}$.

\section{A. Results from ZPPR-15A}

The neutron spectrum measurements made near the core center of ZPPR-15 (matrix location 159-49) were reported earlier in ZPR-TM-469, p. 138. Subsequently, problems were found in the neutron energy calibration. A new calibration was done by matching resonance dips in measured spectra and spectra calculated in an $\mathrm{rz}$ model in 228 neutron energy groups. Fig. 9.1 shows the measured neutron spectrum compared with a 226group spectrum calculated at the center of a one-dimensional diffusion model in the SEF1D module of the SDX code. The calculated spectrum has been smoothed using a Gaussian function for comparison with the finite-resolution detector responses (see ZPR-TM-233).

A comparison in 28 neutron energy groups, between the measured spectrum and a spectrum calculated in an $\mathrm{rz}$ model is shown in Fig. 9.2. Both spectra are normalized over the energy groups between about $2 \mathrm{keV}$ and $2 \mathrm{MeV}$. As Fig. 9.2 shows, there are significant discrepancies between calculation and measurement. Similar, though smaller, differences were observed in the oxide-fuel assembly ZPPR-11 (ANL-RDP-102, p. 357). The first 18 groups of the calculated and measured spectra are also compared in tabular form in Table 9.1. The uncertainties in Table 9.1 are due only to counting statistics. Additional uncertainties of 5 to $10 \%$ are applicable below $10 \mathrm{kev}$ and above $1 \mathrm{MeV}$. Uncertainties in the energy calibration 
for both counters also have correlated components and are estimated to be about $1 \%$ increasing to about $15 \%$ for the range below $2 \mathrm{kev}$ in the hydrogen counter.

The effects of the Gaussian smoothing on the 226 group calculated spectrum are compared in Fig. 9.3. As shown, the effect is to reduce spectral resolution.

The spectrum measurements were made on October 17 and 18, 1985, in reactor loading number 66 , reactor run numbers 140 and 141 . The assembly was about $0.5 \$$ subcritical (all shim rods fully inserted) during the hydrogen counter run and was about $5 \$$ subcritical during the methane counter run (all shim rods and PSRs inserted).

\section{B. Results from ZPPR-15B Core Center}

In $Z P P R-15 B$, the composition of the inner core was changed by substituting zirconium for some of the stainless steel. The ZPPR-15B reference configurations were described in ZPR-TM-470, p. 3. The corecenter neutron energy spectrum measurement was repeated. Figure 9.4 shows the measured spectrum and a 230-group calculated spectrum. The calculated spectrum was obtained as in $Z P P R-15 \mathrm{~A}$, and the difference in group structure was only at energies below $1 \mathrm{keV}$ which has no effect on the results presented here. Again the calculated spectrum was smoothed using a Gaussian technique.

\section{A second comparison in a 21-group structure is shown in}

Fig. 9.5. This group structure was adopted to comply with methods used in the Argonne National Laboratory design group. The calculated spectrum was taken from the reference design methods; nodal diffusion theory in xyz geometry. The normalization is over those groups for which both measured and calculated values are available, although additional calculated group values are shown. The discrepancies between calculation and measurement for ZPPR-15B are very similar to those in ZPPR-15A. 
The first 18 groups of the 21-group spectra are compared in tabular form in Table 9.2. The uncertainties are due to counting statistics only and larger, correlated, uncertainties are appropriate for groups at the high- and low-energy ends of the spectra.

Of primary interest in $Z P P R-15 B$ is the ef fect of zirconium on important physics parameters, including neutron spectrum. The comparison between calculated 21 group spectra in ZPPR-15A and 15B is shown in Fig. 9.6. Only very minor differences are predicted. The comparison between the measured, core-center spectra in the two assemblies is shown in Fig. 9.7. The measured changes are also quite small. The fine-group measurement comparison in shown in Fig. 9.8 .

The spectrum measurements were made on December 3 and 4,1985 , in reactor loading 90 and reactor runs 170 to 172 .

\section{Results from ZPPR-15D Core Center}

In ZPPR-15D, about $90 \%$ of the core drawers contained ${ }^{235} \mathrm{U}$ fuel, compared to $100 \%$ plutonium fuel in $Z P P R-15 A$ and $15 B$. The ZPPR-15D reference configurations are described in ZPR-TM-471, p. 16. The core-center neutron spectrum measurement was repeated.

Figure 9.9 shows the measured spectrum and a 230-group calculated spectrum smoothed by a Gaussian technique. A comparison in 21 energy groups is shown in Fig. 9.10. The calculated spectrum was obtained in a nodal diffusion calculation. Both spectra are normalized over the groups for which both measured and calculated data are available.

The first 18 groups of the 21-group spectra are compared in Table 9.3. The discrepancies between calculation and measurement in ZPPR-15D are very similar to those in assemblies $15 \mathrm{~A}$ and $15 \mathrm{~B}$.

It is of interest to compare the measured and calculated spectra from $Z$ PPR-15D with those from $15 B$ to see the effect of changing from all plutonium to mostly ${ }^{235} \mathrm{U}$ fuel. The calculation comparisons are shown in 
Fig. 9.11 for 230 groups and in Fig. 9.12 for 21 groups. The calculated changes are quite small. The corresponding measurement comparisons are shown in Fig. 9.13 for 230 groups and in Fig. 9.14 for 21 groups. The measured changes are quite small, and are not well correlated with the predicted changes.

\section{Experiments in the ZPPR-15B Shield Zone}

Radiation fields in a mockup of the blanket, reflector, shield and ex-core sodium pool were characterized in a series of experiments in ZPPR15B. These experiments and the special assembly configurations were described in ANL-ZPR-472. Measurements were made for two shield compositions; stainless steel and sodium (SSNA) and $B_{4} C$ and sodium (BCNA).

Results for the measurement outside the SSNA shield are given for 230 groups in Fig. 9.15 and in Fig. 9.16 and Table 9.4 for 21 groups. As for the core, the 230-group spectra were from one-dimensional calculations. The 21-group spectra were from nodal transport calculations in $x y$ geometry. Normalization is over those groups where both calculated and measured fluxes were available. Calculated fluxes for additional groups are shown. Once again, significant discrepancies exist between calculation and experiment.

Results from measurements and calculations outside the BCNA shield are given in Fig. 9.17 for 230 groups and in Fig. 9.18 and Table 9.5 for 21 groups. While the discrepancies between measurement and calculation are still significant, they are substantially less than in the SSNA case. As expected, contributions to the lowest-energy groups are much less in the $B C N A$ case than in the SSNA case.

A comparison of the calculated spectra in the sodium pool for the two different shield compositions is shown in Figs. 9.19 and 9.20. The two calculated spectra are normalized over the same range as the measurement. The $\mathrm{B}_{4} \mathrm{C}$ shield is predicted to yield a harder spectrum outside the shield. A comparison of the measured spectra for the two cases is shown in

Figs. 9.21 and 9.22 . The measurements do not give any information about the 
energy groups below $1 \mathrm{keV}$, but a greater contribution from 0.5 to $1 \mathrm{MeV}$ neutrons is seen in the BCNA case.

The spectrum measurements with the SSNA shield were made January 17, 1986 to January 21, 1986, in loading 121, reactor runs 209 to 211. The assembly configuration was the $Z P P R-15 B$ critical reference modified by the presence of the shield zone, sodium pool and room return shield. A critical configuration was required for ex-core measurements in order to obtain reasonable count rates from the proton recoil chambers. Data were collected with the reactor at a power corresponding to $0.2 \times 10^{-6}$ on EXP1. Measurements with the BCNA shield were made on January 27 and 28 , 1986, in loading 126 and reactor runs 216 to 218. Data were collected with the reactor at a power corresponding to $0.1 \times 10^{-5}$ on EXP1. For both measurements, the proton recoil chambers were in matrix location 158-19. 
TABLE 9.1 Measured and Calculated Fluxes at the Center of ZPPR-15A

\begin{tabular}{|c|c|c|c|c|c|c|}
\hline \multirow{2}{*}{$\frac{\text { Group }}{1}$} & \multicolumn{2}{|c|}{$\begin{array}{c}\text { Energy Bounds } \\
(\mathrm{kev})\end{array}$} & $\begin{array}{c}\text { Normalized } \\
\text { Flux } \\
\text { Measured } \\
\end{array}$ & \multirow{2}{*}{$\begin{array}{l}\text { Statistical } \\
\text { Uncertainty }\end{array}$} & \multirow{2}{*}{$\begin{array}{c}\text { Normalized } \\
\text { Flux } \\
\text { Calculated } \\
0.01573501\end{array}$} & \multirow{2}{*}{$\frac{C / E}{-}$} \\
\hline & 14190.000 & 6065.000 & - & & & \\
\hline 2 & 6065.000 & 3679.000 & - & - & 0.11852640 & - \\
\hline 3 & 3679.000 & 2231.000 & - & - & 0.29567200 & - \\
\hline 4 & 2231.000 & 1353.000 & 0.4399 & 0.0102 & 0.51406240 & 1.1685 \\
\hline 5 & 1353.000 & 820.900 & 0.7403 & 0.0098 & 0.87710250 & 1.1848 \\
\hline 6 & 820.900 & 497.900 & 1.2182 & 0.0094 & 1.43253300 & 1.1759 \\
\hline 7 & 497.900 & 302.000 & 1.6831 & 0.0114 & 1.91459800 & 1.1375 \\
\hline 8 & 302.000 & 183.200 & 1.8858 & 0.0126 & 1.81831700 & 0.9642 \\
\hline 9 & 183.200 & 111.100 & 2.0499 & 0.0130 & 1.85570000 & 0.9053 \\
\hline 10 & 111.100 & 67.380 & 1.6849 & 0.0199 & 1.42128200 & 0.8435 \\
\hline 11 & 67.380 & 40.870 & 1.2320 & 0.0134 & 1.16786000 & 0.9480 \\
\hline 12 & 40.870 & 24.790 & 0.8262 & 0.0141 & 0.76012160 & 0.9200 \\
\hline 13 & 24.790 & 15.030 & 0.7411 & 0.0098 & 0.87834660 & 1.1852 \\
\hline 14 & 15.030 & 9.119 & 0.5263 & 0.0111 & 0.47496700 & 0.9025 \\
\hline 15 & 9.119 & 5.531 & 0.2240 & 0.0063 & 0.20210240 & 0.9022 \\
\hline 16 & 5.531 & 3.355 & 0.1152 & 0.0064 & 0.14585750 & 1.2661 \\
\hline 17 & 3.355 & 2.035 & 0.0725 & 0.0037 & 0.05063323 & 0.6984 \\
\hline 18 & 2.035 & 1.234 & 0.0956 & 0.0032 & 0.15449700 & 1.6167 \\
\hline
\end{tabular}


TABLE 9.2 Measured and Calculated Fluxes at the Center of ZPPR-15B

\begin{tabular}{|c|c|c|c|c|c|c|}
\hline \multirow{2}{*}{$\frac{\text { Group }}{1}$} & \multicolumn{2}{|c|}{$\begin{array}{c}\text { Energy Bounds } \\
(\mathrm{kev})\end{array}$} & $\begin{array}{c}\text { Normalized } \\
\text { Flux } \\
\text { Measured }\end{array}$ & $\begin{array}{l}\text { Statistical } \\
\text { Uncertainty }\end{array}$ & \multirow{2}{*}{$\begin{array}{c}\begin{array}{c}\text { Normalized } \\
\text { Flux } \\
\text { Calculated }\end{array} \\
0.01599614\end{array}$} & \multirow{2}{*}{$\frac{C / E}{-}$} \\
\hline & 14190.000 & 6065.000 & - & - & & \\
\hline 2 & 6065.000 & 3679.000 & - & - & 0.12159340 & - \\
\hline 3 & 3679.000 & 2231.000 & - & - & 0.30011560 & - \\
\hline 4 & 2231.000 & 1353.000 & 0.4531 & 0.0104 & 0.52383120 & 1.1561 \\
\hline 5 & 1353.000 & 820.900 & 0.7496 & 0.0099 & 0.88782160 & 1.1844 \\
\hline 6 & 820.900 & 497.900 & 1.2258 & 0.0096 & 1.43849300 & 1.1735 \\
\hline 7 & 497.900 & 302.000 & 1.6965 & 0.0116 & 1.91861100 & 1.1310 \\
\hline 8 & 302.000 & 183.200 & 1.8646 & 0.0128 & 1.81951600 & 0.9758 \\
\hline 9 & 183.200 & 111.100 & 1.9578 & 0.0131 & 1.84166300 & 0.9407 \\
\hline 10 & 111.100 & 67.380 & 1.6896 & 0.0204 & 1.41300200 & 0.8363 \\
\hline 11 & 67.380 & 40.870 & 1.2643 & 0.0138 & 1.17637600 & 0.9304 \\
\hline 12 & 40.870 & 24.790 & 0.8075 & 0.0146 & 0.78020230 & 0.9662 \\
\hline 13 & 24.790 & 15.030 & 0.7819 & 0.0102 & 0.83960310 & 1.0738 \\
\hline 14 & 15.030 & 9.119 & 0.5470 & 0.0117 & 0.48116410 & 0.8797 \\
\hline 15 & 9.119 & 5.531 & 0.2409 & 0.0068 & 0.20625390 & 0.8562 \\
\hline 16 & 5.531 & 3.355 & 0.1206 & 0.0069 & 0.14720900 & 1.2207 \\
\hline 17 & 3.355 & 2.035 & 0.0534 & 0.0040 & 0.04955604 & 0.9287 \\
\hline 18 & 2.035 & 1.234 & 0.1306 & 0.0035 & 0.15671120 & 1.2000 \\
\hline
\end{tabular}


TABLE 9.3 Measured and Calculated Fluxes at the Center of ZPPR-15D

\begin{tabular}{|c|c|c|c|c|c|c|}
\hline Group & \multicolumn{2}{|c|}{$\begin{array}{l}\text { Energy Bounds } \\
(\mathrm{kev})\end{array}$} & $\begin{array}{l}\text { Normalized } \\
\text { Flux } \\
\text { Measured }\end{array}$ & $\begin{array}{l}\text { Statistical } \\
\text { Uncertainty }\end{array}$ & $\begin{array}{c}\text { Normalized } \\
\text { Flux } \\
\text { Calculated } \\
\end{array}$ & $C / E$ \\
\hline 1 & 14190.000 & 6065.000 & - & - & 0.01434291 & - \\
\hline 2 & 6065.000 & 3679.000 & - & - & 0.11402880 & - \\
\hline 3 & 3679.000 & 2231.000 & - & - & 0.29174790 & - \\
\hline 4 & 2231.000 & 1353.000 & 0.4357 & 0.0110 & 0.52315170 & 1.2008 \\
\hline 5 & 1353.000 & 820.900 & 0.7330 & 0.0105 & 0.89531620 & 1.2214 \\
\hline 6 & 820.900 & 497.900 & 1.2093 & 0.0102 & 1.45806200 & 1.2057 \\
\hline 7 & 497.900 & 302.000 & 1.6758 & 0.0124 & 1.96426600 & 1.1721 \\
\hline 8 & 302.000 & 183.200 & 1.8606 & 0.0137 & 1.87013600 & 1.0051 \\
\hline 9 & 183.200 & 111.100 & 1.9801 & 0.0149 & 1.87884200 & 0.9488 \\
\hline 10 & 111.100 & 67.380 & 1.7124 & 0.0326 & 1.43326600 & 0.8370 \\
\hline 11 & $67 \cdot 380$ & 40.870 & 1.2893 & 0.0222 & 1.17541100 & 0.9117 \\
\hline 12 & 40.870 & 24.790 & 0.7854 & 0.0237 & 0.76263560 & 0.9710 \\
\hline 13 & 24.790 & 15.030 & 0.8052 & 0.0165 & 0.79290260 & 0.9847 \\
\hline 14 & 15.030 & 9.119 & 0.5375 & 0.0192 & 0.43093370 & 0.8017 \\
\hline 15 & 9.119 & 5.531 & 0.2556 & 0.0113 & 0.17688750 & 0.6920 \\
\hline 16 & 5.531 & 3.355 & 0.1089 & 0.0116 & 0.12153000 & 1.1163 \\
\hline 17 & 3.355 & 2.035 & 0.0462 & 0.0068 & 0.03928658 & 0.8511 \\
\hline 18 & 2.035 & 1.234 & 0.1020 & 0.0058 & 0.11758530 & 1.1528 \\
\hline
\end{tabular}


TABLE 9.4 Measured and Calculated Fluxes Outside the Stainless Steel and Sodium Shield in ZPPR-15B

\begin{tabular}{|c|c|c|c|c|c|c|}
\hline Group & $\begin{array}{r}\text { Energ. } \\
(\mathrm{k} \\
\end{array}$ & $\begin{array}{l}\text { Bounds } \\
\text { v) }\end{array}$ & $\begin{array}{c}\text { Normalized } \\
\text { Flux } \\
\text { Measured }\end{array}$ & $\begin{array}{l}\text { Statistical } \\
\text { Uncertainty }\end{array}$ & $\begin{array}{c}\text { Normal ized } \\
\text { Flux } \\
\text { Calculated } \\
\end{array}$ & $\mathrm{C} / \mathrm{E}$ \\
\hline 1 & 14190.000 & 6065.000 & - & - & 0.00002223 & - \\
\hline 2 & 6065.000 & 3679.000 & - & - & 0.00008664 & - \\
\hline 3 & 3679.000 & 2231.000 & - & - & 0.00047164 & - \\
\hline 4 & 2231.000 & 1353.000 & 0.0036 & 0.0007 & 0.00295873 & 0.8120 \\
\hline 5 & 1353.000 & 820.900 & 0.0324 & 0.0012 & 0.02356056 & 0.7281 \\
\hline 6 & 820.900 & 497.900 & 0.1481 & 0.0020 & 0.14522330 & 0.9805 \\
\hline 7 & 497.900 & 302.000 & 0.3912 & 0.0036 & 0.38976370 & 0.9964 \\
\hline 8 & 302.000 & 183.200 & 0.8817 & 0.0054 & 0.86355940 & 0.9794 \\
\hline 9 & 183.200 & 111.100 & 1.4423 & 0.0077 & 1.25938900 & 0.8732 \\
\hline 10 & 111.100 & $67 \cdot 380$ & 1.8062 & 0.0218 & 1.33999400 & 0.7419 \\
\hline 11 & $67 \cdot 380$ & 40.870 & 1.6497 & 0.0169 & 1.42487800 & 0.8637 \\
\hline 12 & 40.870 & 24.790 & 1.2899 & 0.0200 & 1.19163200 & 0.9238 \\
\hline 13 & 24.790 & 15.030 & 1.7357 & 0.0155 & 2.21125100 & 1.2740 \\
\hline 14 & 15.030 & 9.119 & 1.3423 & 0.0198 & 1.60804000 & 1.1979 \\
\hline 15 & 9.119 & 5.531 & 0.8213 & 0.0126 & 0.88989320 & 1.0835 \\
\hline 16 & 5.531 & 3.355 & 0.4152 & 0.0135 & 0.49003370 & 1.1803 \\
\hline 17 & 3.355 & 2.035 & 0.0755 & 0.0078 & 0.18546710 & 2.4577 \\
\hline 18 & 2.035 & 1.234 & 0.6040 & 0.0069 & 1.19504200 & 1.9785 \\
\hline 19 & 1.234 & 0.454 & - & - & 1.21505000 & - \\
\hline 20 & 0.454 & 0.061 & - & - & 0.75742160 & - \\
\hline 21 & 0.061 & 0.000 & - & - & 0.13412330 & - \\
\hline
\end{tabular}


TABLE 9.5 Measured and Calculated Fluxes Outside the $B_{4} C$ and Sodium Shield in ZPPR-15B

\begin{tabular}{|c|c|c|c|c|c|c|}
\hline Group & \multicolumn{2}{|c|}{$\begin{array}{c}\text { Energy Bounds } \\
(\mathrm{kev})\end{array}$} & $\begin{array}{c}\text { Normal ized } \\
\text { Flux } \\
\text { Measured } \\
\end{array}$ & $\begin{array}{l}\text { Statistical } \\
\text { Uncertainty }\end{array}$ & $\begin{array}{c}\text { Normalized } \\
\text { Flux } \\
\text { Calculated }\end{array}$ & $\mathrm{C} / \mathrm{E}$ \\
\hline 1 & 14190.000 & 6065.000 & - & - & 0.00129106 & - \\
\hline 2 & 6065.000 & 3679.000 & - & - & 0.00638425 & - \\
\hline 3 & 3679.000 & 2231.000 & - & - & 0.01916176 & - \\
\hline 4 & 2231.000 & 1353.000 & 0.0522 & 0.0037 & 0.05956990 & 1.1417 \\
\hline 5 & 1353.000 & 820.900 & 0.1696 & 0.0043 & 0.14872490 & 0.8770 \\
\hline 6 & 820.900 & 497.900 & 0.3904 & 0.0054 & 0.34910860 & 0.8941 \\
\hline 7 & 497.900 & 302.000 & 0.6054 & 0.0074 & 0.55450520 & 0.9159 \\
\hline 8 & 302.000 & 183.200 & 0.8578 & 0.0086 & 0.90057160 & 1.0498 \\
\hline 9 & 183.200 & 111.100 & 1.3054 & 0.0108 & 1.38821700 & 1.0635 \\
\hline 10 & 111.100 & 67.380 & 1.6140 & 0.0273 & 1.46651700 & 0.9086 \\
\hline 11 & 67.380 & 40.870 & 1.5973 & 0.0211 & 1.50774600 & 0.9439 \\
\hline 12 & 40.870 & 24.790 & 1.4019 & 0.0258 & 1.45090500 & 1.0349 \\
\hline 13 & 24.790 & 15.030 & 1.5536 & 0.0199 & 1.62441500 & 1.0456 \\
\hline 14 & 15.030 & 9.119 & 1.3331 & 0.0252 & 1.34685000 & 1.0103 \\
\hline 15 & 9.119 & 5.531 & 0.8381 & 0.0160 & 0.80593210 & 0.9617 \\
\hline 16 & 5.531 & 3.355 & 0.4215 & 0.0173 & 0.45288840 & 1.0746 \\
\hline 17 & 3.355 & 2.035 & 0.0809 & 0.0099 & 0.15175030 & 1.8760 \\
\hline 18 & 2.035 & 1.234 & 0.4397 & 0.0087 & 0.83038950 & 1.8884 \\
\hline 19 & 1.234 & 0.454 & - & - & 0.46217300 & - \\
\hline 20 & 0.454 & 0.061 & - & - & 0.05928639 & - \\
\hline 21 & 0.061 & 0.000 & - & - & 0.00022800 & - \\
\hline
\end{tabular}




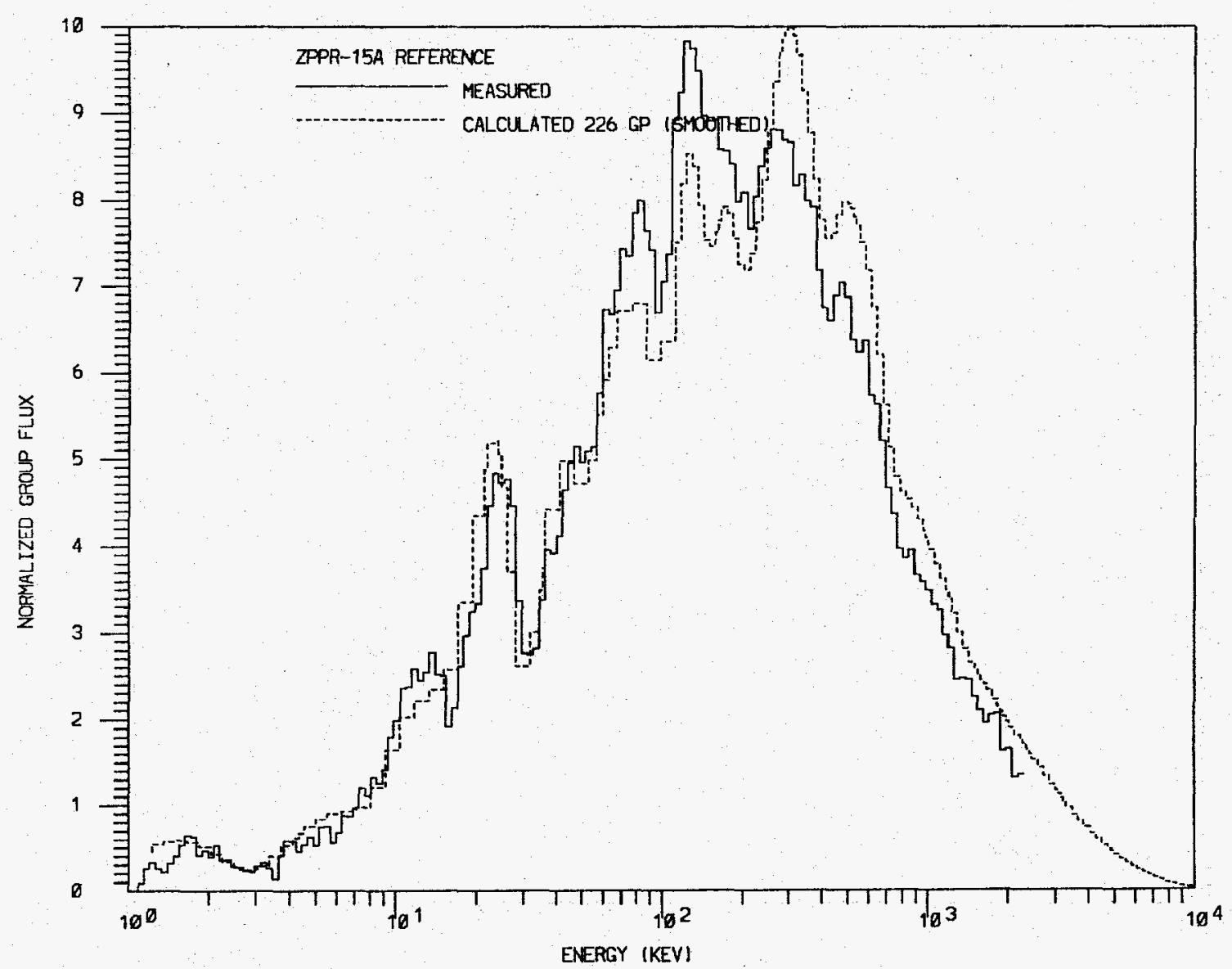

Fig. 9.1. Measured and Calculated 226-Group Spectrum at the ZPPR-15A Core Center. 


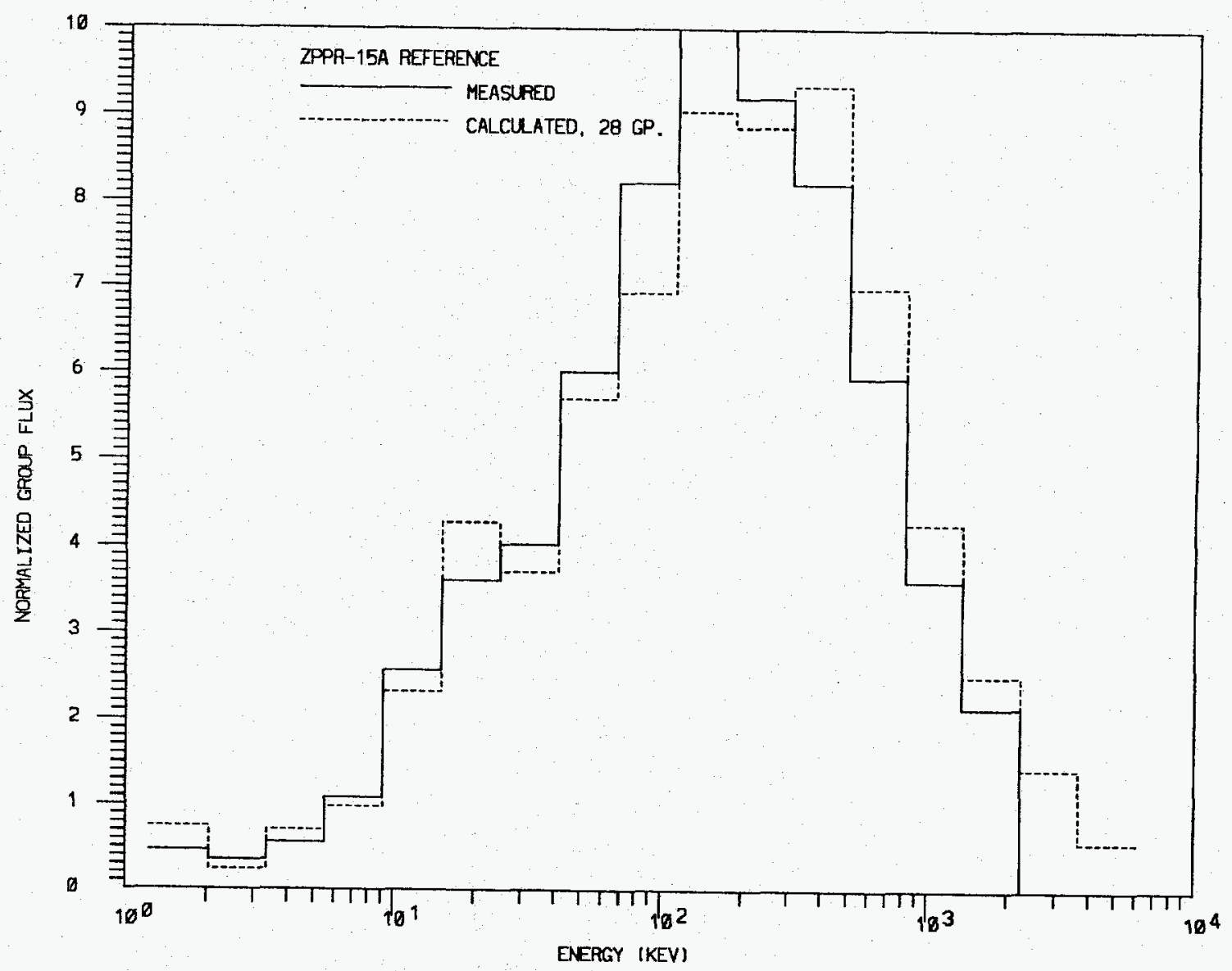

Fig. 9.2. Measured and Calculated 28-Group Spectra at the ZPPR-15A Core Center. 


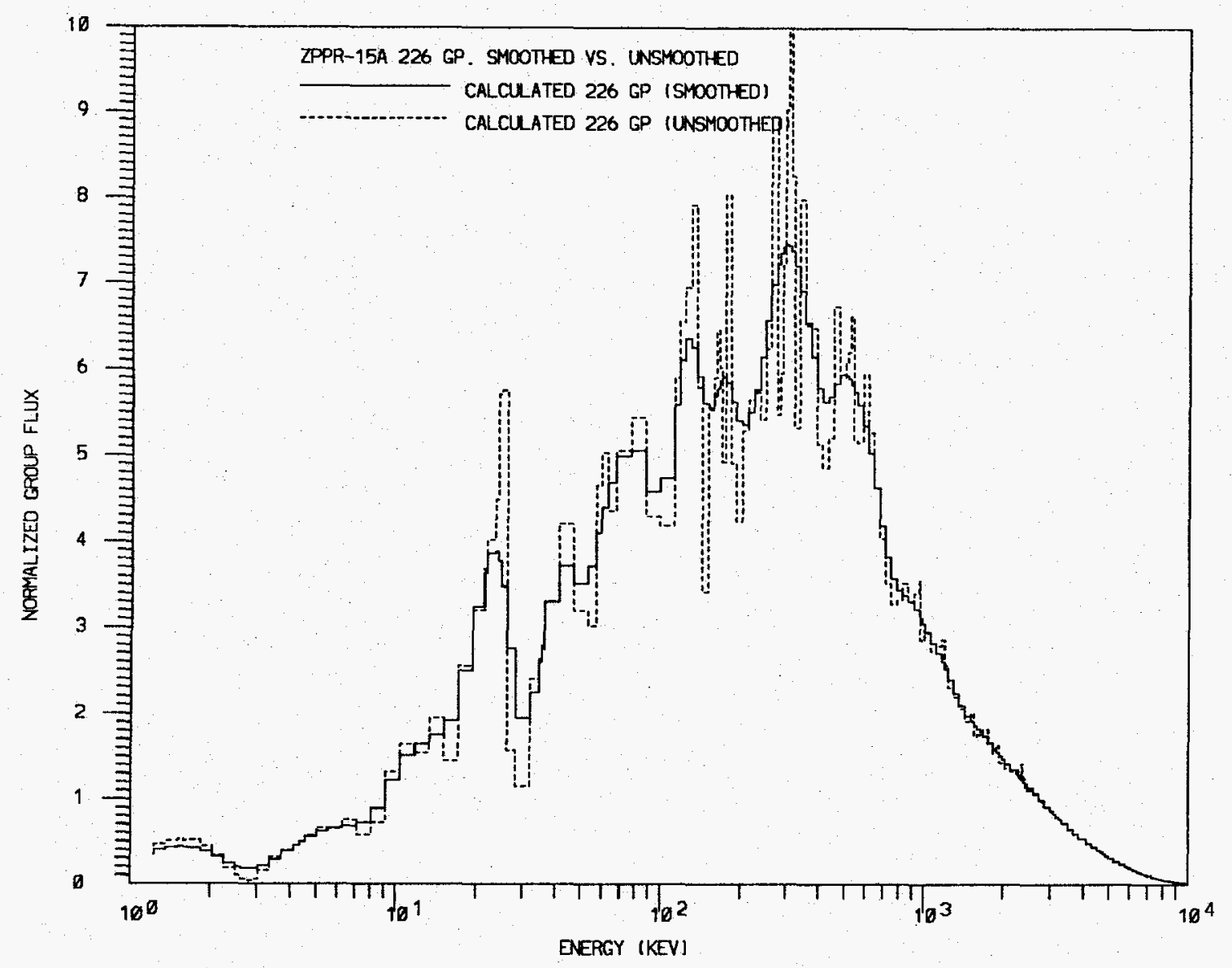

Fig. 9.3. Effects of Gaussian Smoothing on ZPPR-15A Core Spectrum. 


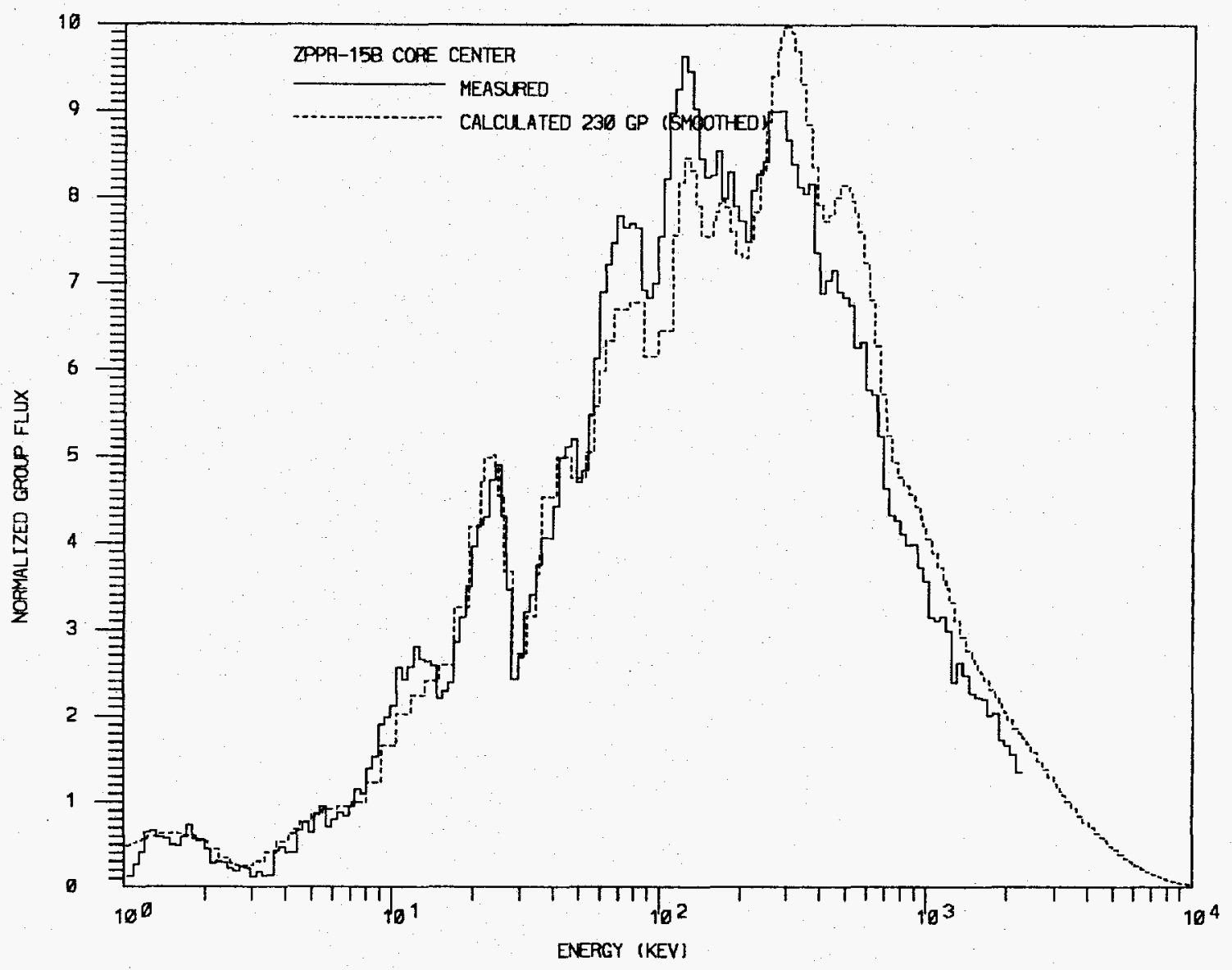

Fig. 9.4. Measured and Calculated 230-Group Spectra at the ZPPR-15B Core Center. 


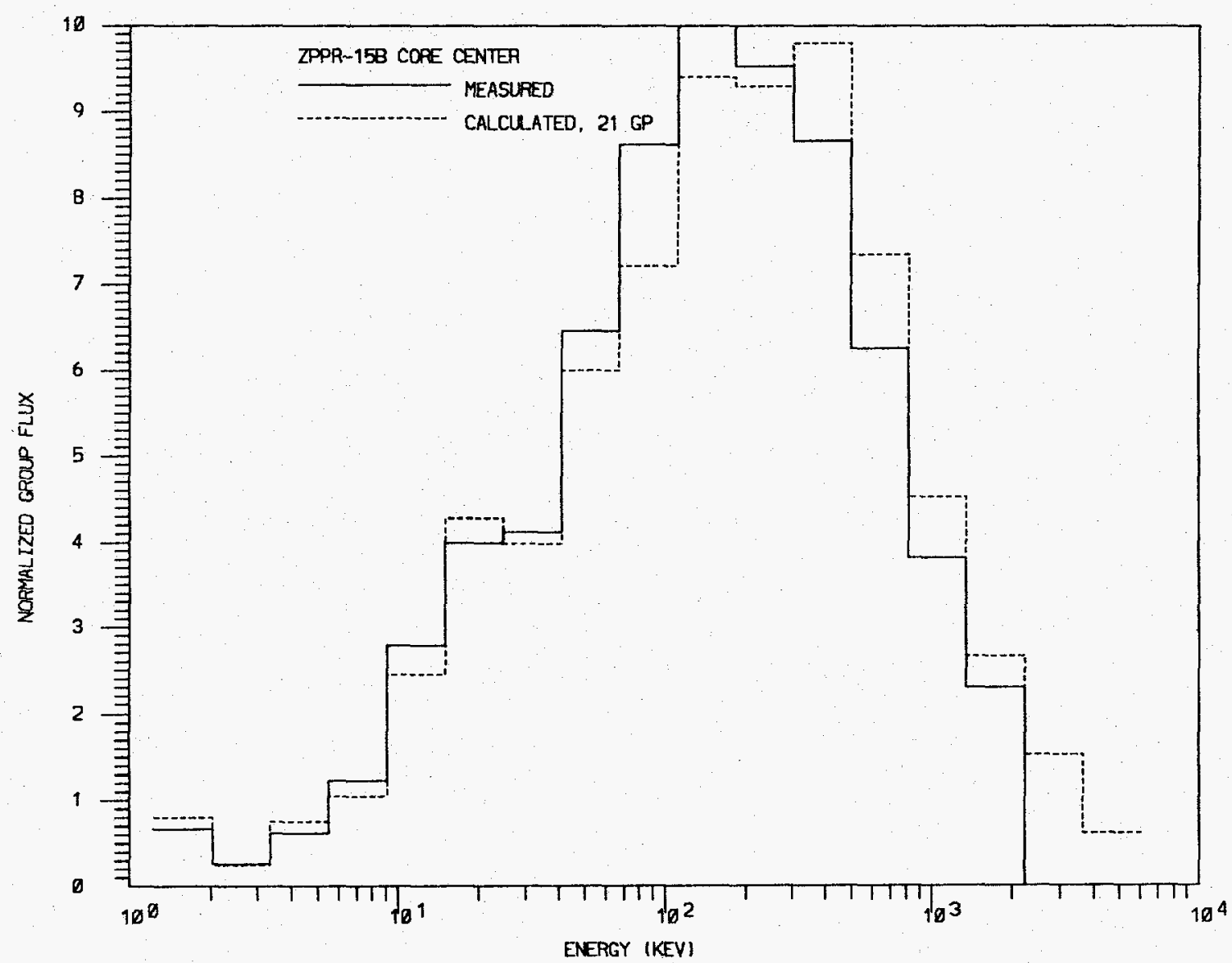

FIg. 9.5. Measured and Calculated 21-Group Spectra in the ZPPR-15B Core Center. 


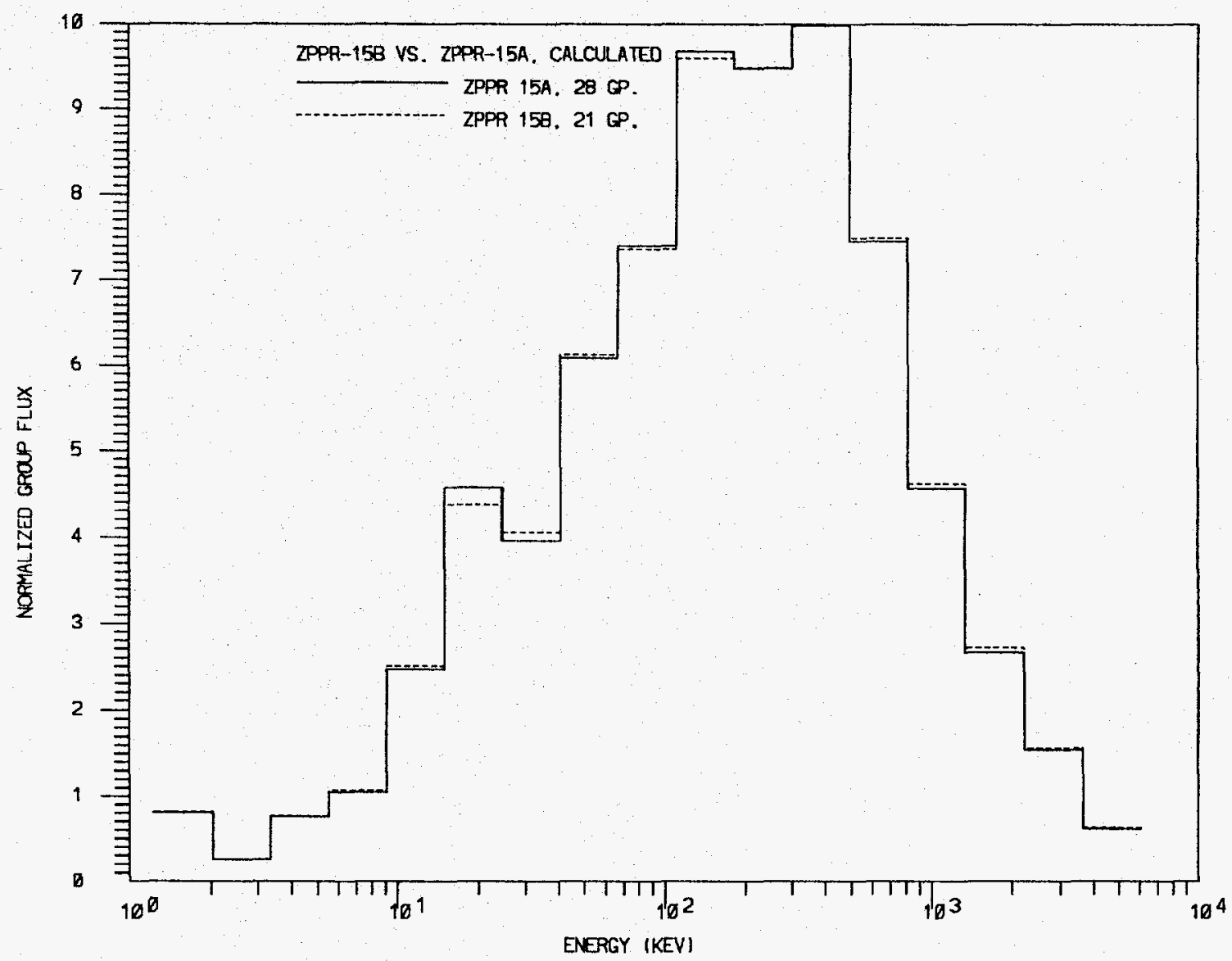

Fig. 9.6. Comparison between 21-Group Calculated Spectra in ZPPR-15A and ZPPR-15B. 


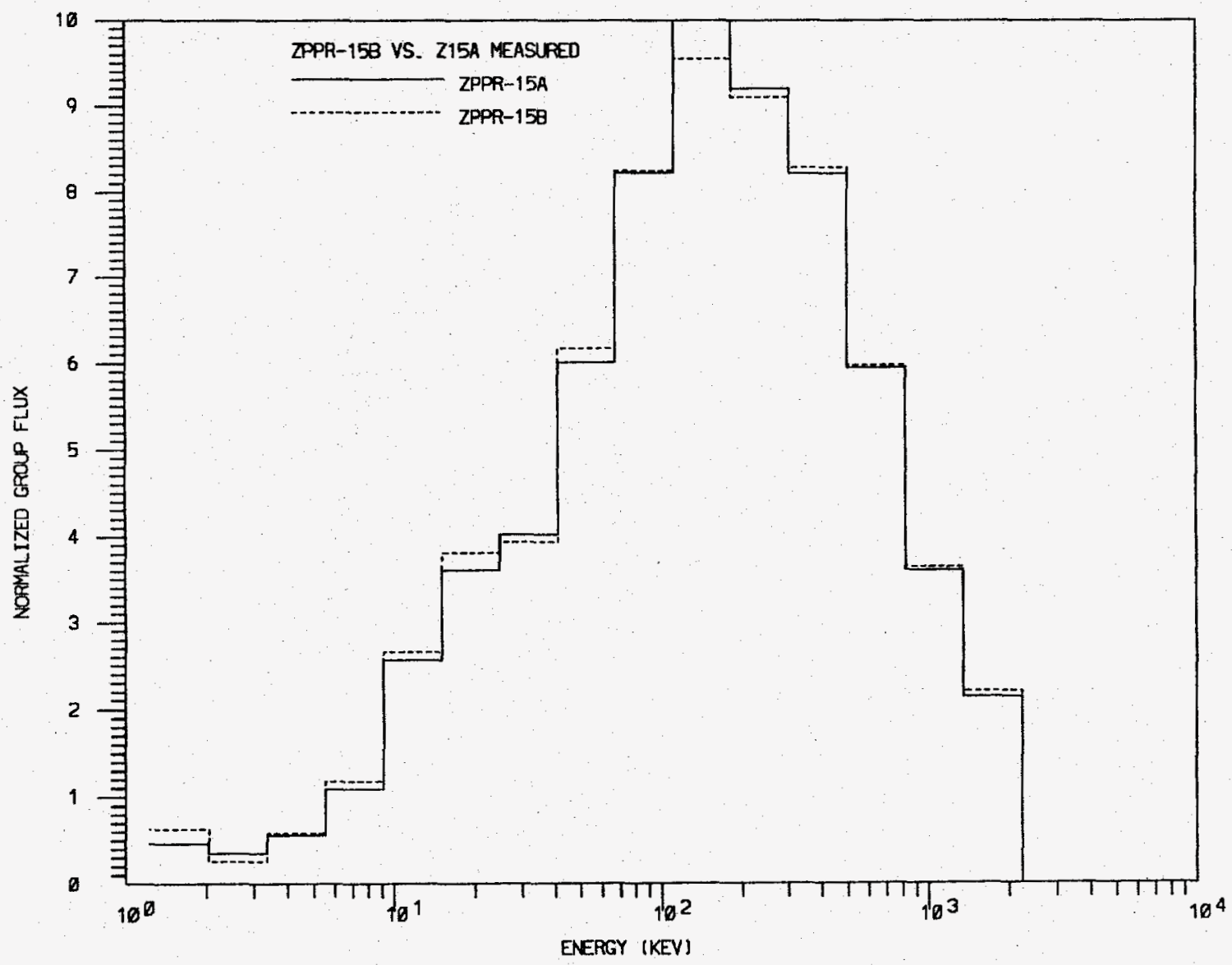

Fig. 9.7. Comparison between 21-Group Measured Spectra in ZPPR-15A and ZPPR-15B. 


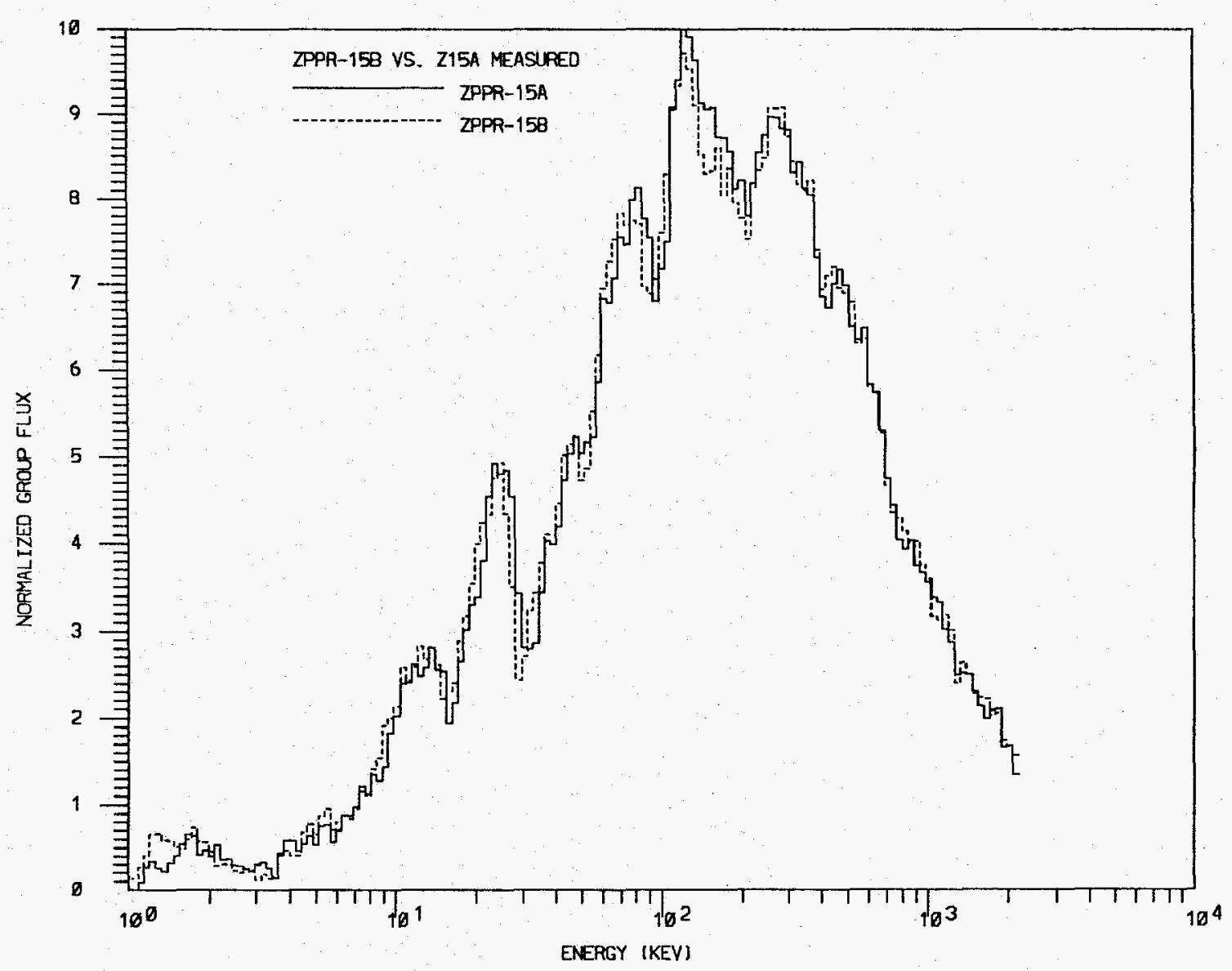

Fig. 9.8. Comparison between 230-Group Measured Spectra in ZPPR-15A and ZPPR-15B. 


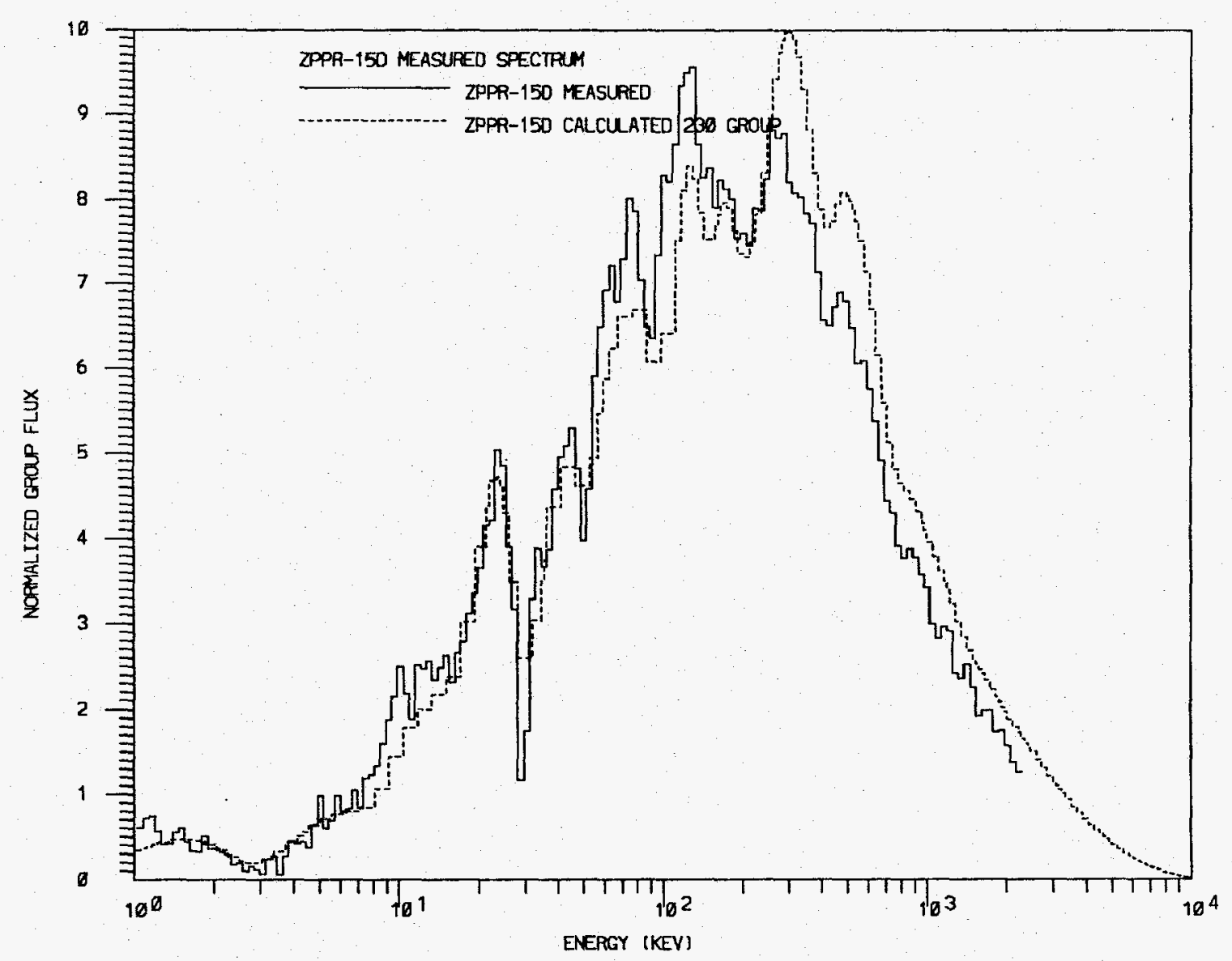

Fig. 9.9. Measured and Calculated 230-Group Spectra in the ZPPR-15D Core Center. 


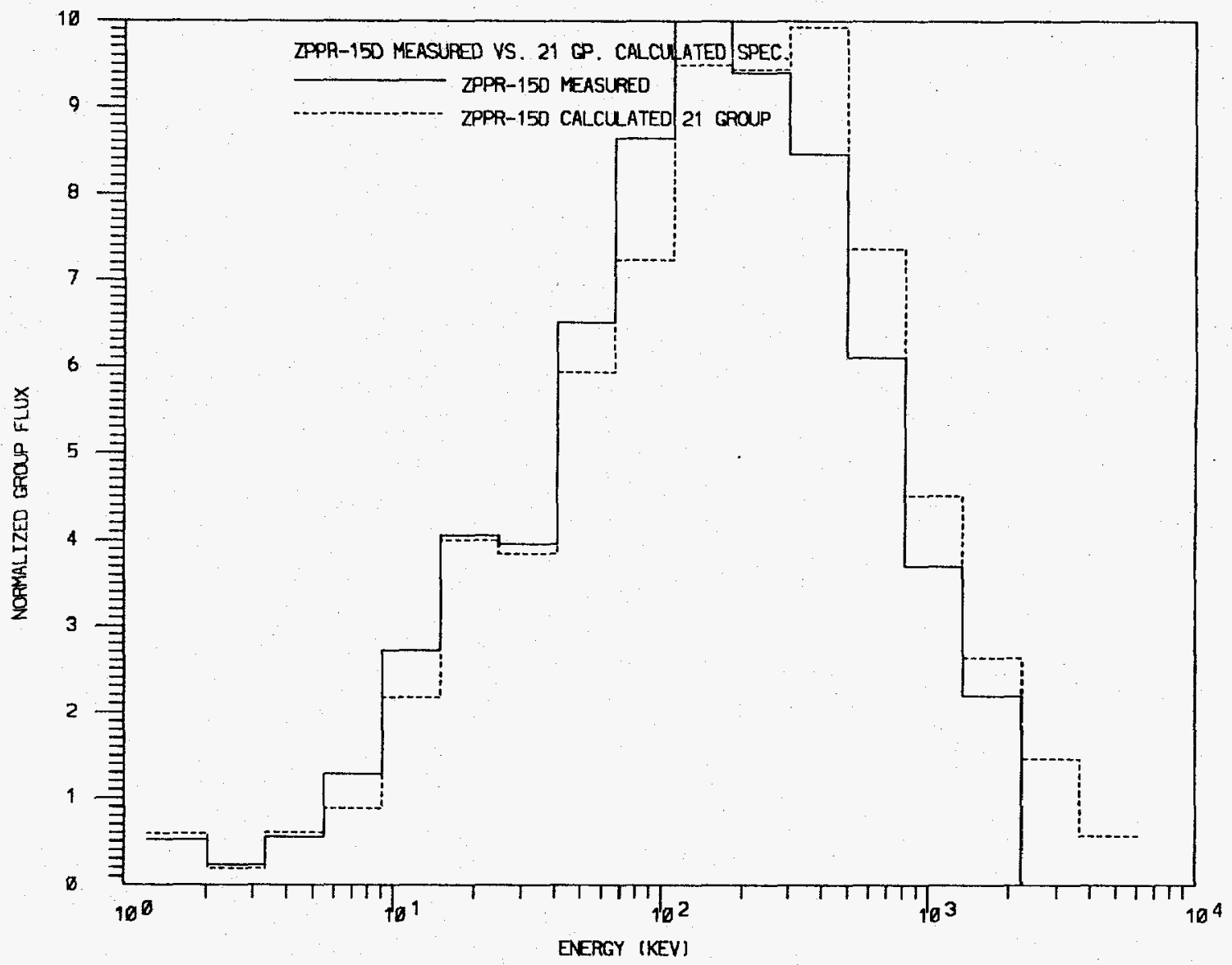

Fig. 9.10. Measured and Calculated 21-Group Spectra in the ZPPR-15D Core Center. 


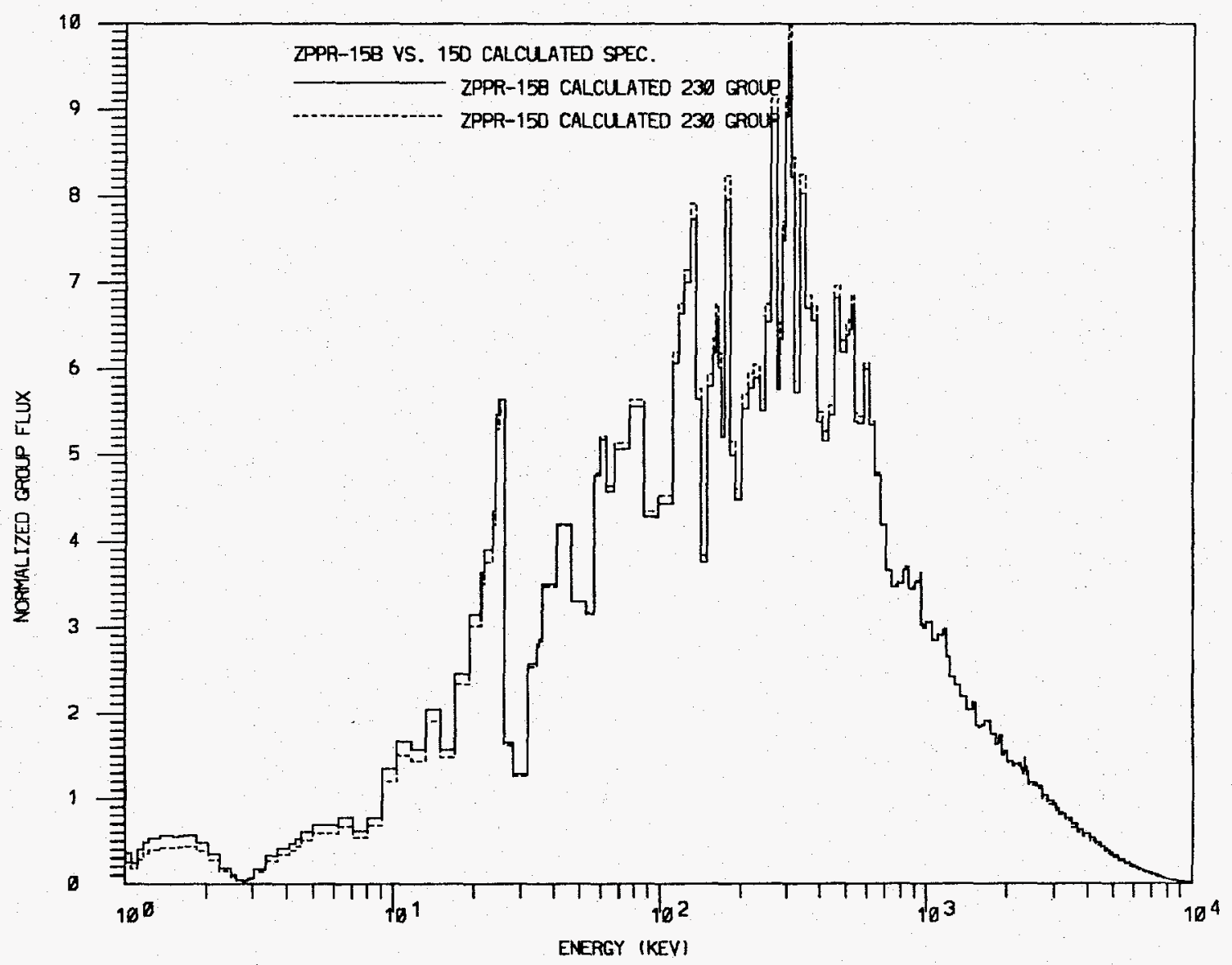

Fig. 9.11. Comparison between 230-Group Calculated Spectra in ZPPR-15B and ZPPR-15D. 


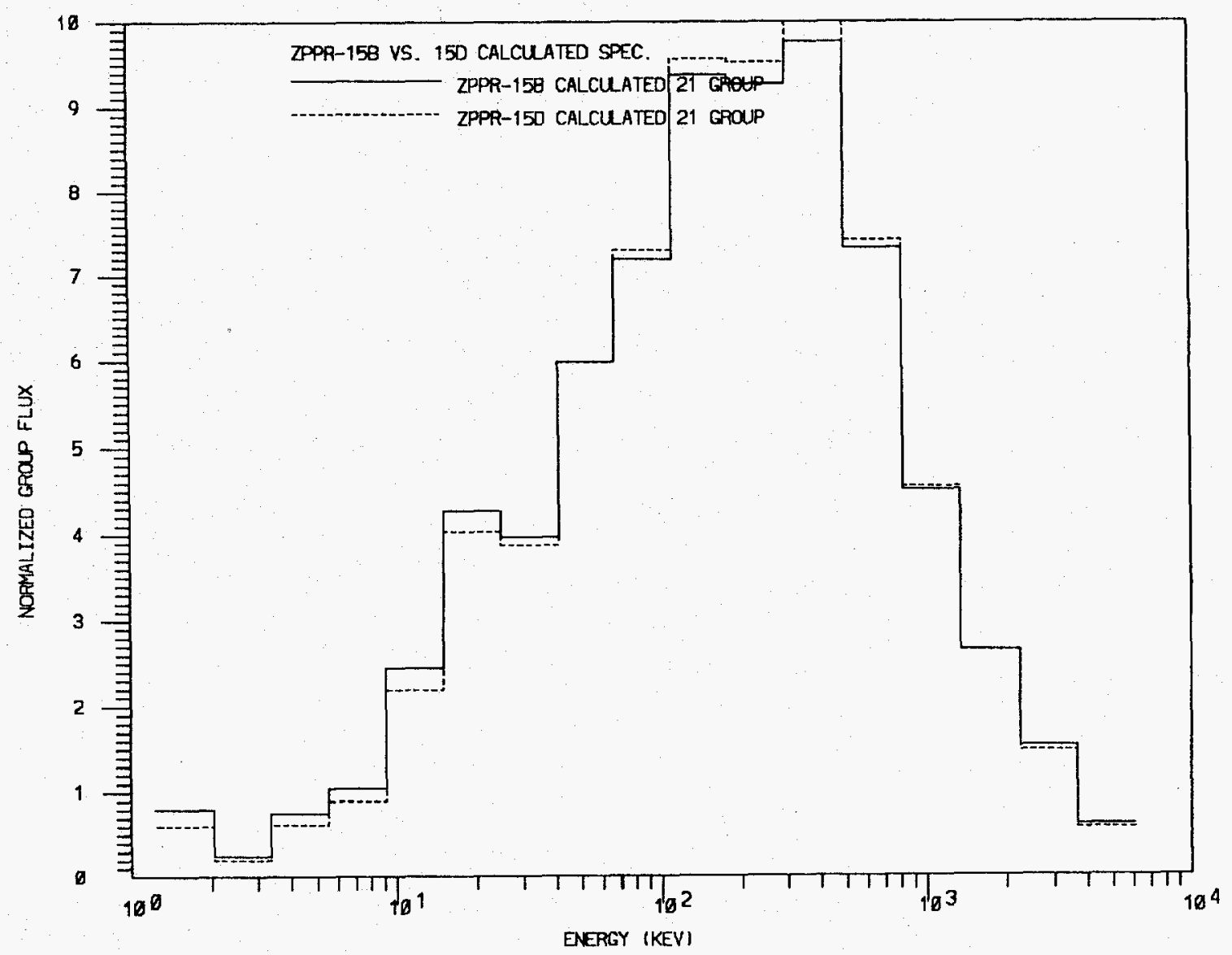

Fig. 9.12. Comparison between 21-Group Calculated Spectra in ZPPR-15B and ZPPR-15D. 


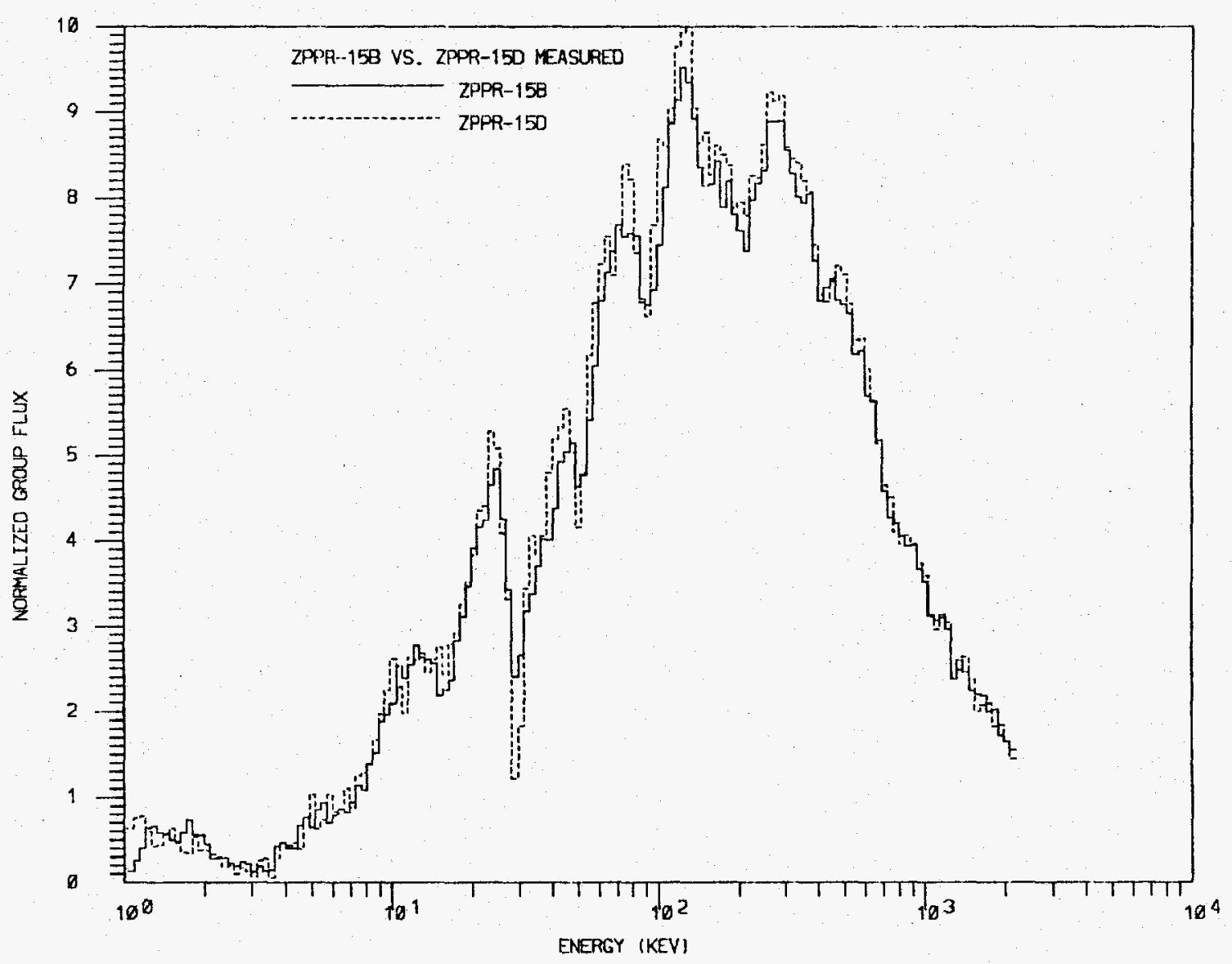

Fig. 9.13. Comparison between 230-Group Measured Spectra in ZPPR-15B and ZPPR-15D. 


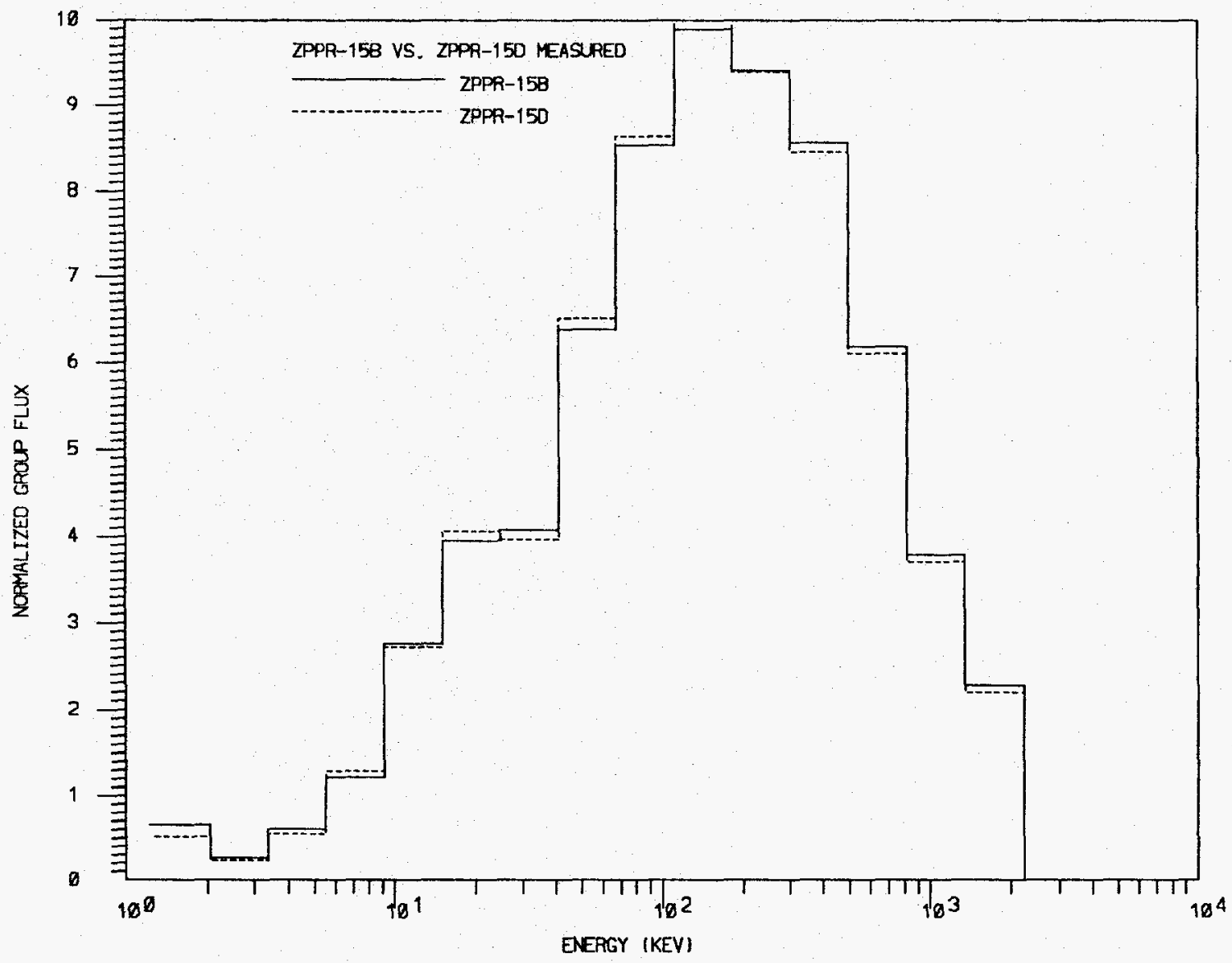

Fig. 9.14, Comparison between 21-Group Measured Spectra in ZPPR-15B and ZPPR-15D. 


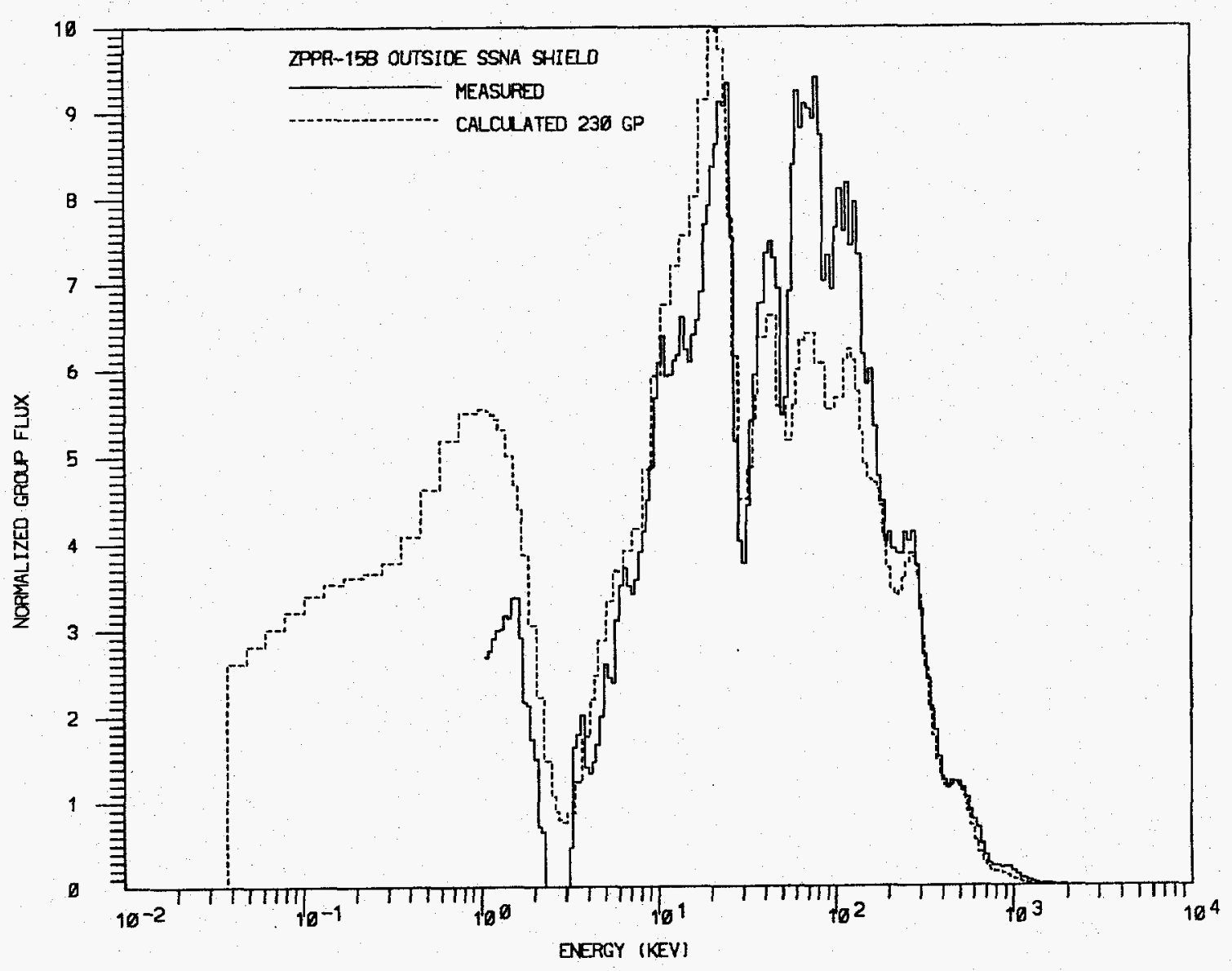

F1g. 9.15. Measured and Calculated 230-Group Spectra Outside the Stainless Steel and Sodium Shield in ZPPR-15B. 


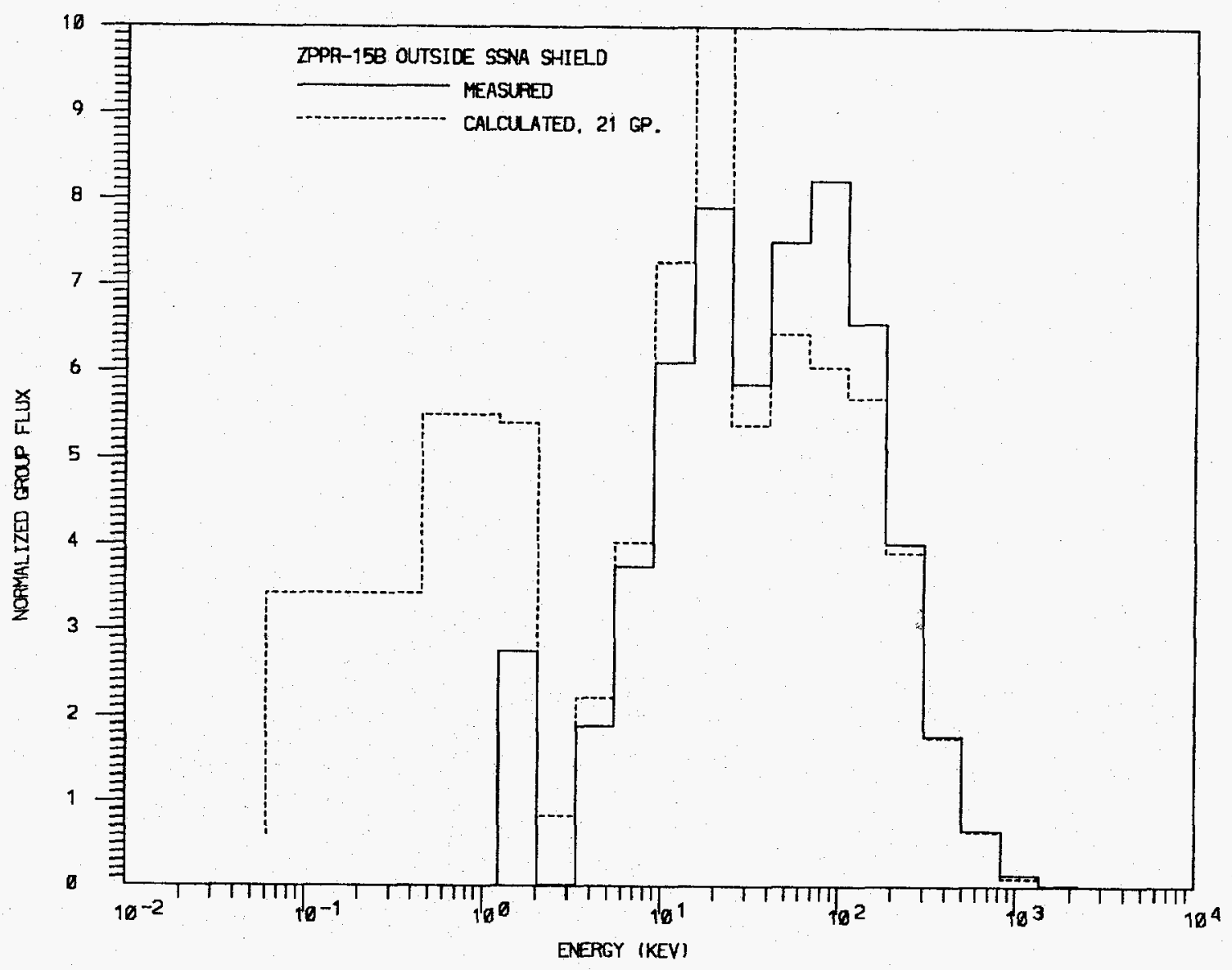

Fig. 9.16. Measured and Calculated 21-Group Spectra Outside the Stainless Steel and Sodium Shield in ZPPR-15B. 


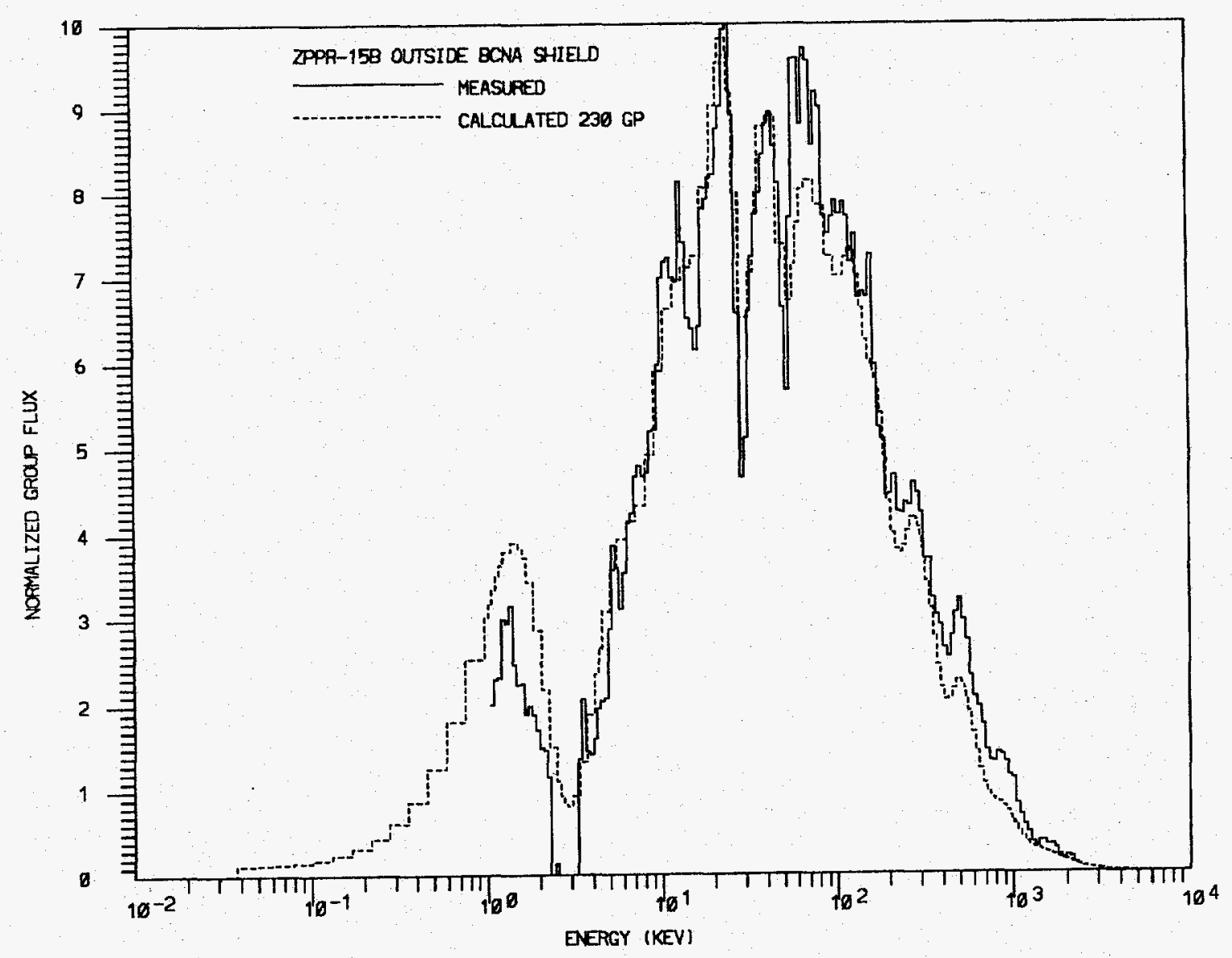

Fig. 9.17. Measured and Calculated 230-Group Spectra Outside the $\mathrm{B}_{4} \mathrm{C}$ and Sodium Shield in ZPPR-15B. 


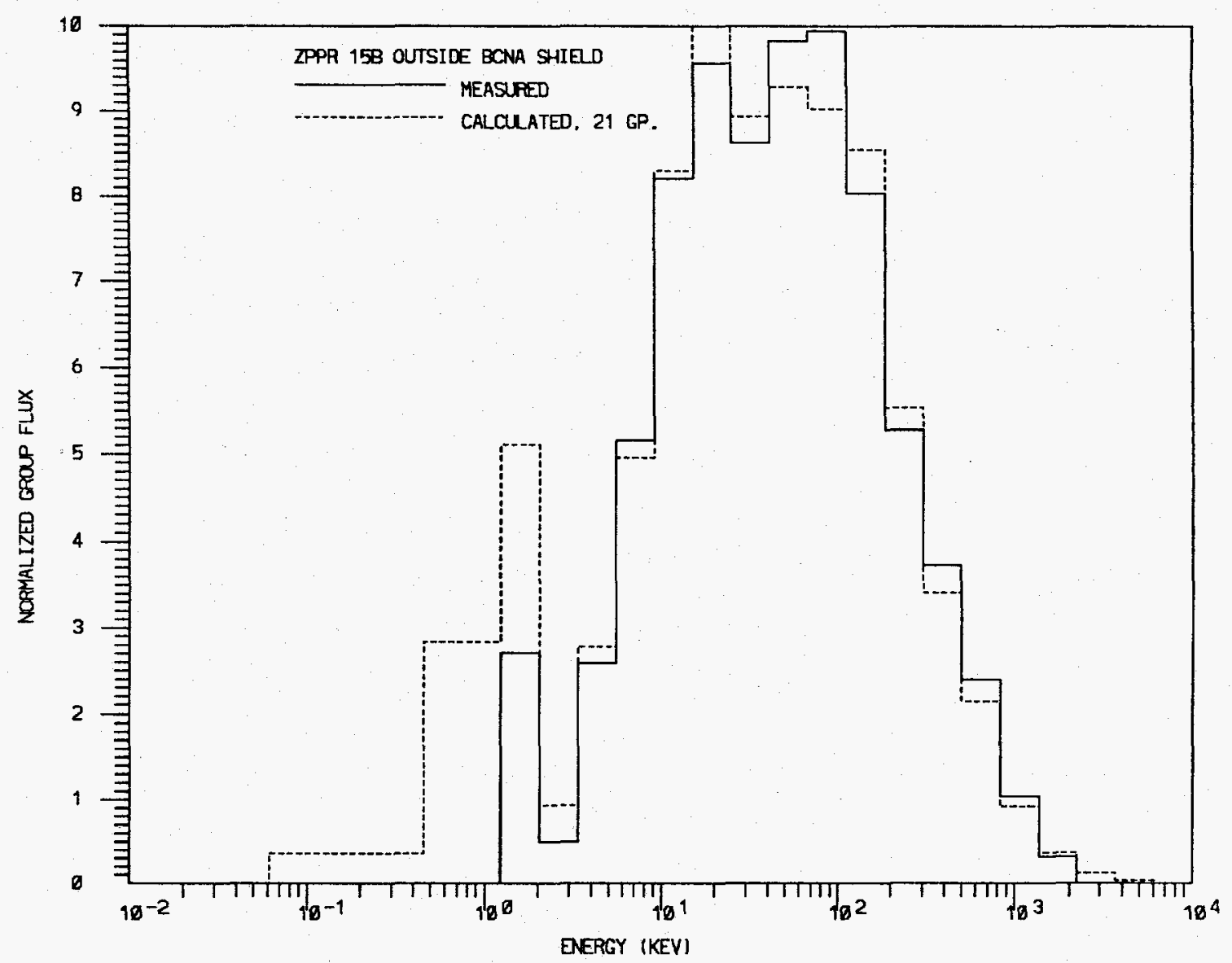

Fig. 9.18. Measured and Calculated 21-Group Spectra Outside the $\mathrm{B}_{4} \mathrm{C}$ and Sodium Shield in ZPPR-15B. 


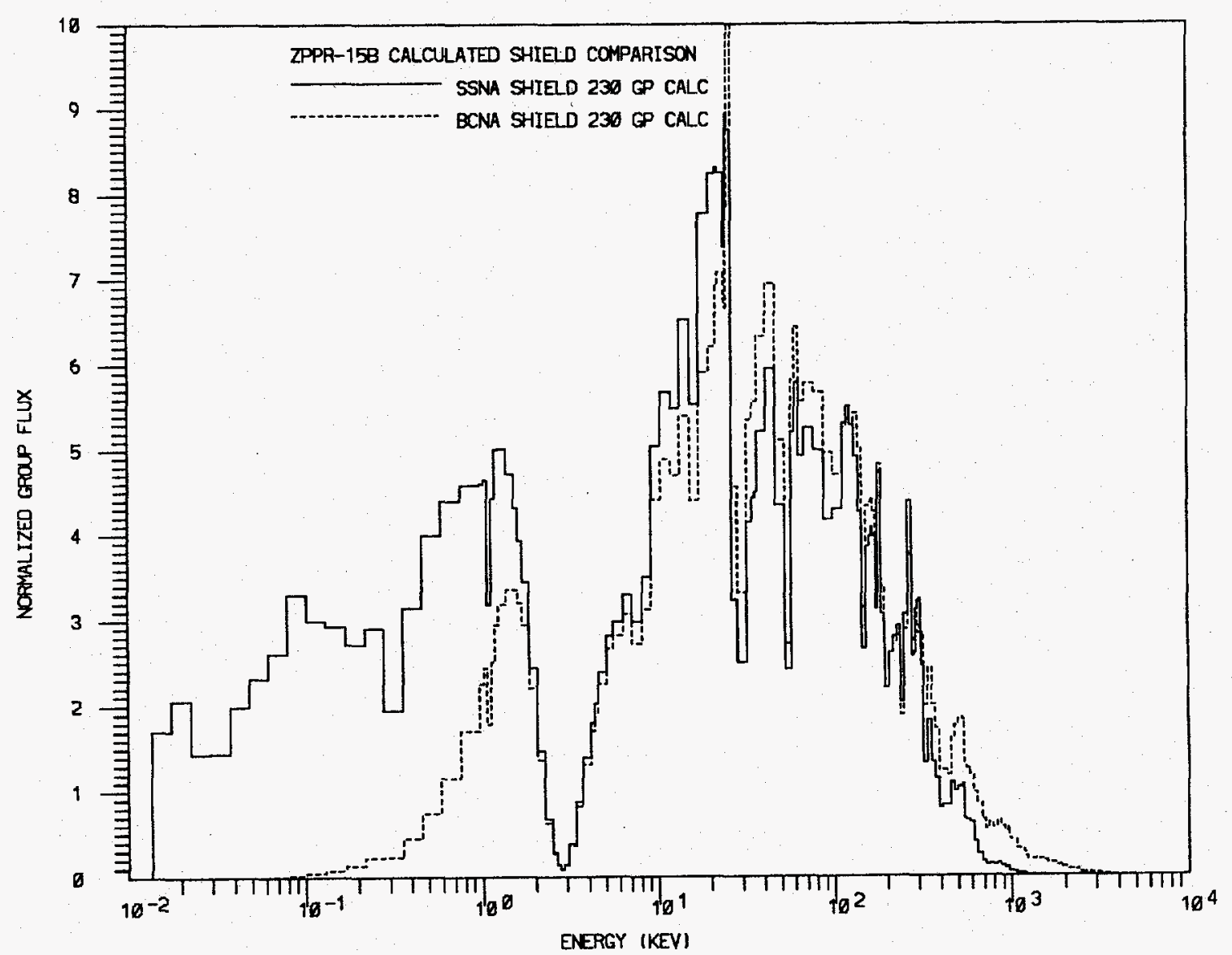

Fig. 9.19. Comparison between 230-Group Calculated Spectra Outside and SSNA and BCNA Shields. 


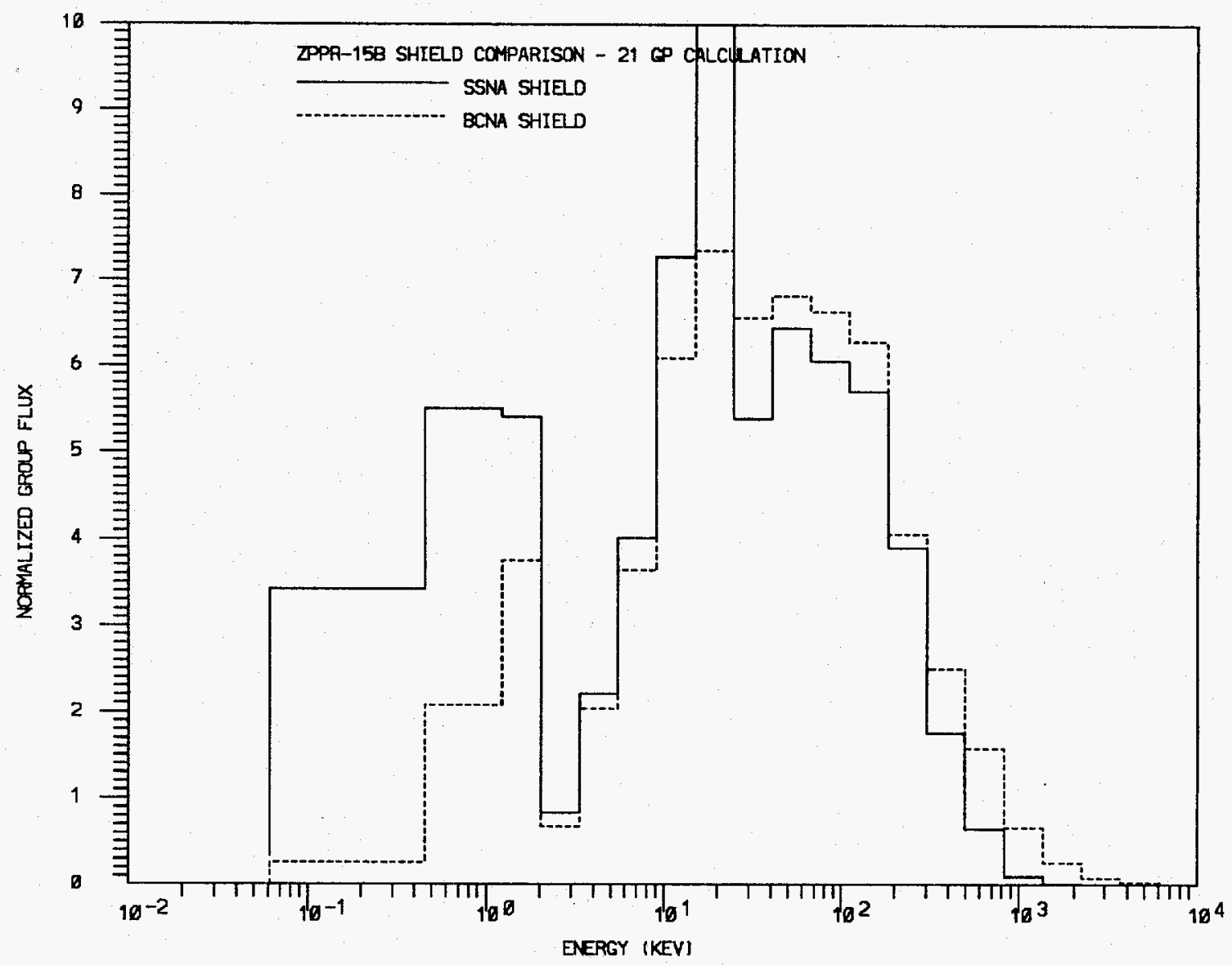

Fig. 9.20. Comparison between 21-Group Calculated Spectra Outside the SSNA and BCNA Shields. 


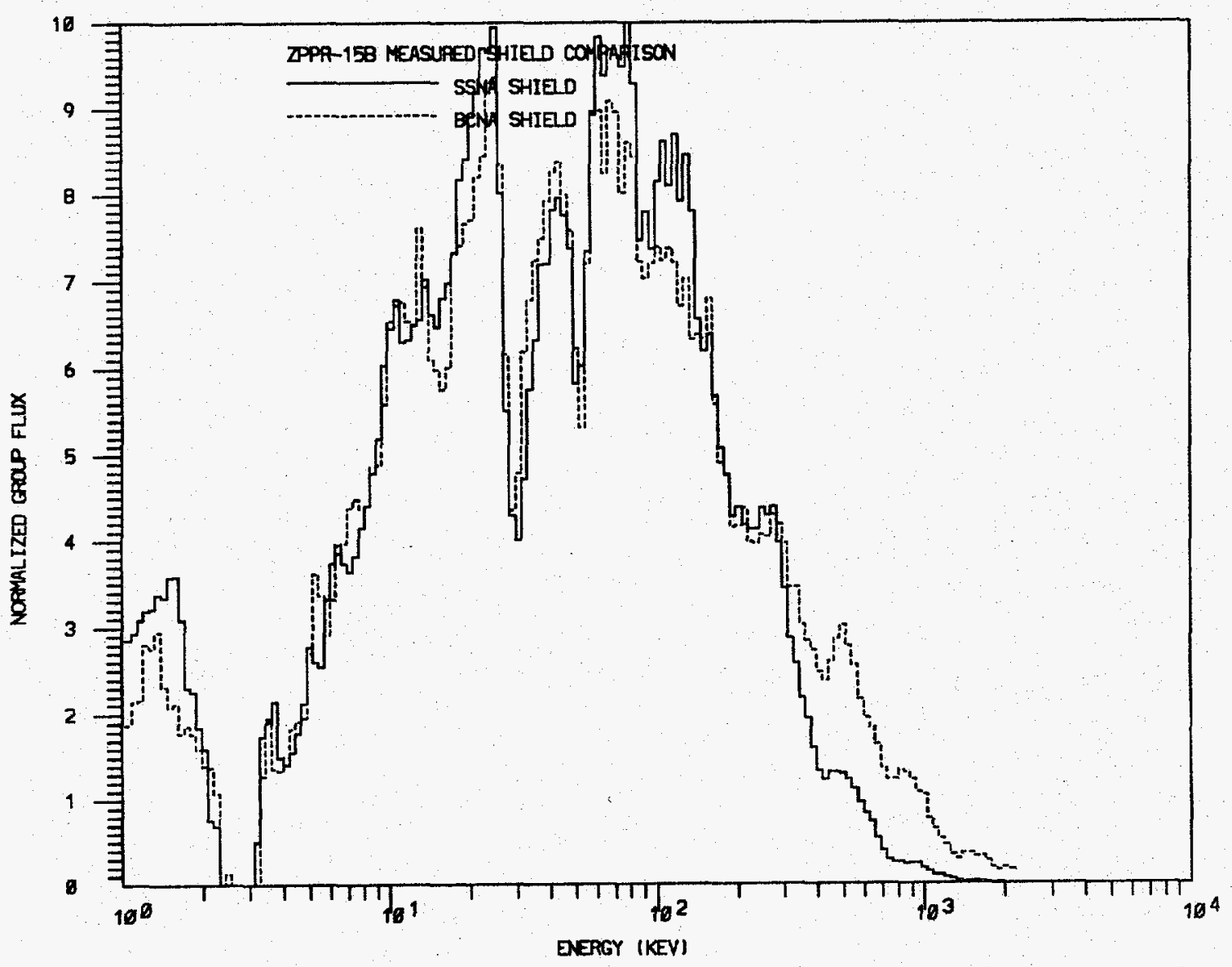

FIg. 9.21. Comparison between 230-Group Measured Spectra Outside and SSNA and BCNA Shields. 


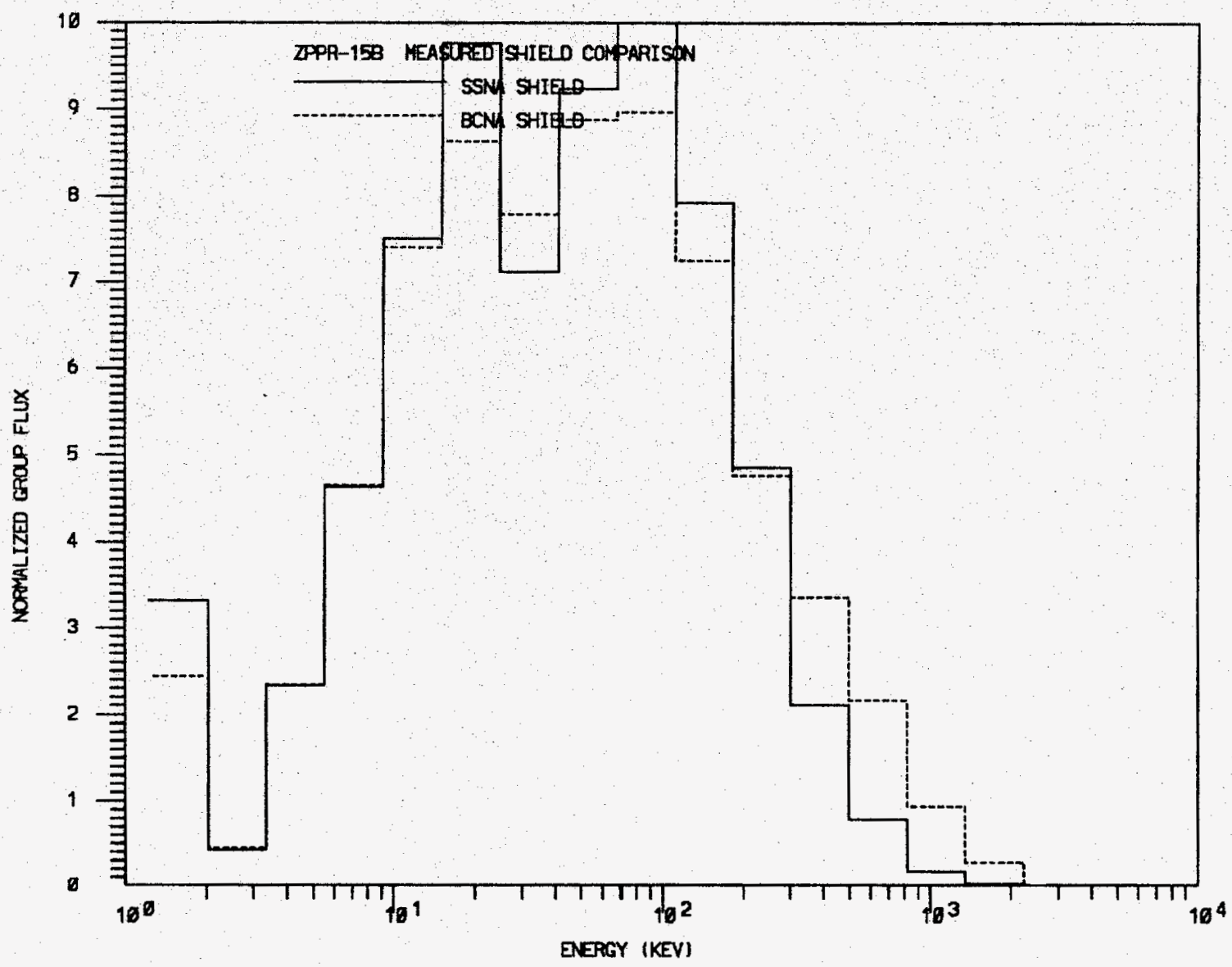

Fig. 9.22. Comparison between 21-Group Measured Spectra Outside the SSNA and BCNA Shields. 
10. CALCULATIONS OF THE CONTROL DRIVELINE EXPANSION SIMULATION IN ZPPR-13D (R. W. Schaefer)

One of the feedback effects that must be predicted in translent calculations for the current innovative LMR designs is control driveline expansion. This feedback occurs due to different axial expansion of the core and control driveline in slow transients. Early in the transient the net expansion is such that the control rod tips move a small distance further into the core and later the motion reverses.

In the most recent designs the cores are made to have a minimal reactivity swing over the burnup cycle. Consequently the rod tips are always near the core-blanket interface where the react ivity effect from expansion is relatively small. In earlier reactor designs the control rods were deeply Inserted into the core at the beginning of the fuel cycle, leading to a more substantlal feedback effect.

Design calculations treat this feedback using a constant react irity coefflcient. In the Applied Physics Division's deslgn section the coefficient is determined from the elgenvalue change resulting from Inserting the control rod bank one or two cm. beyond its nominal position. Two elgenvalue calculations are performed using finite-difference diffusion theory. The mesh spacing is identical in the two calculations; it has 6 triangles per hex in the hex plane and is $5 \mathrm{~cm}$ axially, except where the rod tip motion occurs.

For the experiment analysis we have followed this design procedure. One mesh per drawer was used in the XY plane, whlch corresponds approximately to 6 triangles per hex. The 21 group cross seotion set used was produced by C. A. Atkinson from ENDF/B Version 5.2 data. The beta-effective value used, 0,003365 , is based on ENDF/B Version 5 delayed neutron data. The convergence criterion for the elgenvalue was made very smal1, $10^{-8}$ to assure adequate precision in the calculated results. The rod motion worth in cents was computed as $\left(k_{2}-k_{1}\right) * 100 /\left(k_{1} *_{2} *_{B}-e f f e c t i v e\right)$. 
The experiments were reported in ANL-ZPR-476, p. 81. A bank of 6 control rods was mocked up in fuel ring 2 of ZPPR-13D. Starting with the rod tips $10.2 \mathrm{~cm}$ ( 4 inches) Into the Half-1 axial blanket, the bank was inserted in steps, and the assembly subcrlticality was measured at each step. The insertion steps were $2.5 \mathrm{~cm}$ ( 1 inch) Increments until the tips were $10.2 \mathrm{~cm}$ into the core, and then a few large steps were used to reach full insertion. The $2.5 \mathrm{~cm}$ steps are the ones that best simulate expansion.

Because the full-z model, elgenvalue calculations required to analyze these exper iments are very expensive, only a few of the measurement steps were calculated. The calculated positions are $0.00,2.54,7.62,10.16$ and $91.44 \mathrm{~cm}$, where 0.00 corresponds to the the rod bank tips being at the core-blanket interface. The reactivity for insertion from 0.00 to $2.54 \mathrm{~cm}$ gives the coeffloient for the bank parked at the core/blanket interface. The worth gradient from 7.62 to $10.16 \mathrm{~cm}$ is close to the maximum insertion worth gradient. The full insertion $(0.0$ to $91.44 \mathrm{~cm})$ worth was computed to see whether the total rod bank worth C/E for this configuration is typical.

In preparation for getting the expansion coefficlents, the rod bank insertion worths were determined. Data and results for the insertion worths are shown in Table 10.1 . It is clear that the calculational error at the core-blanket boundary is different from the error for deeper insertions.

Another interesting result is that, except for the first step, the insertion worth C/Es are smaller than is typlcal for full insetion worths. To see whether this is due to mesh and transport errors, the full insertion worth was recomputed using nodal transport theory. The eigenvalues went up considerably ( to 0.995273 for $0.00 \mathrm{~cm}$ and 0.975180 for $91.44 \mathrm{~cm}$ ) and the insertion worth $C / E$ Increased almost $4 \%$ to 0.935 . Thus the full insertion worth prediction has been Improved by accounting for mesh and transport errors, but a significant discrepancy remains.

Control expansion reactivity coefflelents are shown in table 10.2. The coefficlent increases by almost a factor of 2 over the $10 \mathrm{~cm}$ from the core-blanket boundary. F18, 8.2 in ANL-ZPR-476 shows that by $10 \mathrm{~cm}$ from this boundary the worth slope (coefficlent) has almost reached a constant 
value. Near the boundary, however, the slope is changing most rapidiy. Comparing the first two experimental coefficients in Table 10.2 it can be seen that the coefficient changes by $10 \%$ over the two $2.54 \mathrm{~cm}$ intervals that bracket the boundary. This indicates the range of validity of the constant coefficient assumption made in the design calculations.

The C/E for the coefficients varies by about $25 \%$. There is not a uniform $C / E$ trend with distance from the core-blanket boundary, but more data points are needed to clarify this behavior. It is likey that the $\mathrm{C} / \mathrm{E}$ variation is related to the inaccuracy of diffusion theory near material boundaries. Nodal transport calculations were not attemped because the accuracy of this method, as implemented in the DIF3D code, deteriorates when small mesh intervals (which are needed to model these experiments) are used.

A factor to consider in trying to generalize these results for design applications is the extent to which $Z P P R-13 D$ is an appropriate testbed. This was a mixed oxide-fueled, radially heterogeneous assembly. More importantly it was very loosely coupled azimuthally. Consequently many parameters in this assembly, perhaps including these expansion coefficients, are unusually sensitive to methods and modeling errors. It is 1 kely that $\mathrm{C} /$ Es from a more typical core would be at least as close to unity as the C/Es found here. Clearly it would be desirable to confirm this. Considering that the control driveline expansion is one of the weaker feedback mechanisms in the most recent core designs, the calculational accuracy observed here may be considered adequate. 
TABLE 10.1 Rod Bank Insertion Worth Data

\begin{tabular}{|c|c|c|c|c|c|}
\hline Rod Bank Insertion ${ }^{a}(\mathrm{~cm})$ & 0.00 & 2.54 & 7.62 & 10.16 & 91.44 \\
\hline Experimental Worth ${ }^{\mathrm{b}}(\phi)$ & $0.00 \pm 0.00$ & $-6.49 \pm 0.20$ & $-25.70 \pm 0.38$ & $-37.57 \pm 0.48$ & $-662.79 \pm 6.36$ \\
\hline Eigenvalue & 0.990601 & 0.990329 & 0.989870 & 0.989476 & 0.971490 \\
\hline Boron Density Factor ${ }^{c}$ & 1.0000 & 0.9997 & 1.0040 & 1.0036 & 1.0069 \\
\hline Calculated Worth ( $(4)$ & 0.00 & -6.92 & -22.25 & -34.25 & -594.26 \\
\hline $\mathrm{C} / \mathrm{E}^{\mathrm{b}}$ & -- & $1.066 \pm 0.033$ & $0.866 \pm 0.013$ & $0.912 \pm 0.012$ & $0.897 \pm 0.009$ \\
\hline
\end{tabular}

$a_{0.00}$ corresponds to rod tips at the Half -1 core-blanket interface.

${ }^{b}$ The uncertainties include a statistical component and a much larger, correlated component.

${ }^{c}$ Ratio of boron density in actual drawer master for this rod bank position to density in calculational model. This factor is included in the calculated worth. 
TABLE 10.2 Control Driveline Expansion Reactivity Coefficients

\begin{tabular}{lccccc} 
Position Change $(\mathrm{cm})$ & -2.54 to 0.00 & 0.00 to 2.54 & 2.54 to 7.62 & 7.62 to 10.16 \\
E $(\phi / \mathrm{cm})$ & $-2.32 \pm 0.06$ & $-2.56 \pm 0.08$ & $-3.78 \pm 0.07$ & $-4.67 \pm 0.19$ \\
$\mathrm{C}(\phi / \mathrm{cm})$ & -3 & -2.72 & -3.02 & -4.72 \\
$\mathrm{C} / \mathrm{E}$ & - & $1.066 \pm 0.033$ & $0.798 \pm 0.016$ & $1.011 \pm 0.041$ \\
\hline
\end{tabular}

